\title{
NEURAL NETWORK BASED INCIPIENT FAULT DETECTION OF INDUCTION MOTORS
}

CENTRE FOR NEWFOUNDLAND STUDIES

TOTAL OF 10 PAGES ONLY MAY BE XEROXED

(Without Author's Permission)

\section{MOHD. ROKONUZZAMAN}




Neural Network Based Incipient Fault Detection of Induction Motors

\author{
By \\ -Mohd. Rokonuzzaman, B.Sc. Eng.
}

A THESIS SUMBMITTED TO THE SCHOOL OF GRADUATE STUDIES IN PARTIAL FULFILMENT OF THE REQUIREMENTS FOR. THE DEGREE OF MASTER OF ENGINEERING.

FACULTY OF ENGINEERING AND APPLIED SCIENCE. MEMORIAL UNIVERSITY OF NEWFOUNDLAND.

MARCH, 1995 
National Library

of Canada

Acquisitions and Bibliographic Services Branch

395 Welington Street

Ottawa, Onlario

KIA ON 4
Bibliotheque nationale

du Canada

Direction des acquisitions et

des senvices bibliographiques

395, ne Welington

Otrawa (Ontario)

KIACN4

Vout to varefolurence

The author has granted an irrevocable non-exclusive licence allowing the National Library of Canada to reproduce, loan, distribute or sell copies of his/her thesis by any means and in any form or format, making this thesis available to interested persons.

The author retains ownership of the copyright in his/her thesis. Neither the thesis nor substantial extracts from it may be printed or otherwise reproduced without his/her permission.
L'auteur a accordé une licence irrévocable et non exclusive permettant à la Bibliothèque nationale du Canada de reproduire, prêter, distribuer ou vendre des copies de sa thèse de quelque manière et sous quelque forme que ce soit pour mettre des exemplaires de cette thèse à la disposition des personnes intéressées.

L'auteur conserve la propriété du droit d'auteur qui protège sa thèse. Ni la thèse ni des extraits substantiels de celle-ci ne doivent être imprimés ou autrement reproduits sans son autorisation.

ISBN $\quad 0-612-01914-4$ 


\begin{abstract}
An incipient fault detection scheme of induction motors through the recognition of frequency spectra of the stator current has been developed in this thesis. It is based on the adaptive resonance theory of neural networks. This fault diagnosis scheme is not only capable of detecting a fault but also can report if it cannot identify a particular fault so that necessary preventive steps can be taken to update the underlying neural network to adapt to this undetected fault. Moreover, it can update itself to cope with this dynamic situation retaining already acquired knowledge without the need of retraining with the old patterns.

A laboratory experimental set-up using a digital signal processing(DSP) technique has been employed to collect the frequency spectra of the stator current at different fault conditions. A wound-rotor induction motor has been used as the test motor to create different types of faults making unbalance in the stator and rotor circuits. A 24-bit high speed DSP board has been used with a personal computer to develop a real-time interactive software to collect the spectra. A driver for the HP-plotter has also been developed to directly plot the frequency spectra of the stator current .

Adaptive resonance theory(ART) based network is a recent addition to the neural network family. A new software has been successfully developed and implemented in the laboratory experiment using ART neural network. Its performances in training, recalling and dynamic updating have been studied with a set of example patterns. The incipient faults of a 3-phase wound rotor induction motor have been successfully diagonized by this neural network.
\end{abstract}




\section{Acknowledgements}

I would like to express my sincere gratitude to my thesis supervisor, Professor M.A. Rahman for his important contribution to this work. It is through his patience, understanding and advice that this work has been done.

Special thanks are due to technical staff at the faculty of Engineering and Applied Science for their assistance in the experimental part of the thesis. I would like to extend my thanks to other researchers working in the Power Research Laboratory at the Memorial University of Newfoundland for their kind co-operation.

1 am indebted to the Government of Canada as well as the Government of Bangladesh to provide financial and other technical assistances to carry out my graduate study. 1 wish to thank the officials of the Canadian International Development Agency(CIDA), Mcmorial University of Newfoundland(MUN) and Bangladesh Institute of Technology(BIT), Rajshahi for their respective assistances. Particular thanks to Professor M.A. Rahman, Director, CIDA/MUN/BIT project.

Finally, I owe it to my dear family for their patience, encouragement and blessings when I am thousands of miles away from them. 


\section{Contents}

1 Introduction 1

1.1 Incipient Fault Detection of Induction Motors . . . . . . 2

1.1.1 A Brief Description . . . . . . . . . . . 2

1.1.2 Mathematical Analysis of Induction Motor . . . . . 4

1.2 Artificial Neural Network for Fault Detection . . . . . . 7

1.2.1 Expert Approach for Fault Detection . . . . . . . 7

1.2.2 Learning Skills of Artificial Neural Networks . . . . . 8

1.3 Literature Review . . . . . . . . . . . . . . . 8

1.4 Objective of the Present Work . . . . . . . . . 12

1.5 Overview of Thesis $\ldots \ldots \ldots \ldots \ldots \ldots \ldots$

2 An overview of Artificial Neural Networks 14

2.1 Introduction $\ldots \ldots \ldots \ldots \ldots \ldots \ldots \ldots$

2.1.1 Models of a Neuron . . . . . . . . . . . . 15

2.1.2 Network Architecture . . . . . . . . . . . 17

2.1.3 Artificial Intelligence and Neural Networks . . . . . 18

2.2 Backpropagation $\ldots \ldots \ldots \ldots \ldots \ldots \ldots \ldots$ 
2.3 The Binary Associative Memory(BAM) and the Hopfield Mem-

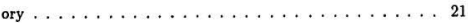

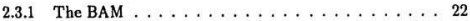

2.3.2 The Hopfield Memory . . . . . . . . . . . 24

2.4 Simulated Annealing . . . . . . . . . . . 25

2.4.1 The Boltzman Machine . . . . . . . . . . . 25

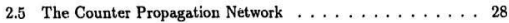

2.5.1 CPN Building Blocks . . . . . . . . . . . . 29

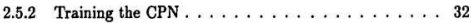

2.5.3 Forward Mapping . . . . . . . . . . . 33

2.6 Self-Organizing Maps . . . . . . . . . . . . . . 34

2.6.1 Unit Activations . . . . . . . . . . 35

2.6.2 The SOM Learning Algorithm . . . . . . . . 35

2.7 Spatiotemporal Pattern Classification . . . . . . . 35

2.7.1 The Formal Avalanche . . . . . . . . . . . . 36

2.7.2 Architectures of Spatiotemporal Networks(STNS) . . 36

2.8 The Neocognitron . . . . . . . . . . . . . . . 37

2.8.1 Neocognitron Architecture . . . . . . . . . . 37

2.8.2 Neocognitıon Data Processing . . . . . . . . . . . 38

2.9 Adaptive Resonance Theory(ART) . . . . . . . . 39

2.9.1 ART Network Description . . . . . . . . . . 39

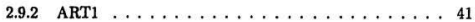

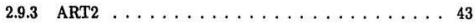

2.10 Comparative analysis and selection of suitable Network . . . 43 
3 ART2 Neural Network $\quad 45$

3.1 Introduction $\ldots \ldots \ldots \ldots \ldots \ldots \ldots \ldots \ldots$

3.2 ART2 Architecture . . . . . . . . . . . . . . 45

3.2.1 The Attentional Subsystem $\ldots \ldots \ldots \ldots \ldots$

3.2.2 The Orienting Subsystem . . . . . . . . . . . 49

3.2.3 Gain Control in ART2 . . . . . . . . . . . . . 50

3.2.4 Least-mean-square Equations . . . . . . . . . 51

3.2.5 Bottom-Up Least-mean-square Initialization . . . . . 52

3.2.6 ART2 Processing Summary . . . . . . . . . . . 52

3.3 ART2 Simulator . . . . . . . . . . . . 55

3.3.1 Model of ART2 as an Object . . . . . . . . . 55

3.3.2 Modified Structure of Training and Recalling Pattern in the Network . . . . . . . . . . . . . 55

3.3.3 Dynamic Updating . . . . . . . . . . 56

3.4 Experimental Varification of Performance . . . . . . . 56

3.4.1 Training of the Neural Network with test Patttern . . . 58

3.4.2 Dynamic Neuron Addition . . . . . . . . . 60

3.4.3 Pattern Recall from the Network . . . . . . . . 61

4 Fault Related Information Collection 63

4.1 Introduction $\ldots \ldots \ldots \ldots \ldots \ldots \ldots \ldots$

4.2 Model of Spectra Collection . . . . . . . . . . . 63

4.3 Discrete Time Signals and Systems . . . . . . . . . 64

4.3.1 Discrete-Time Signals: Sequences . . . . . . . . . 65

4.3.2 Discrete-Time Systems . . . . . . . . . . . 65 
4.3.3 Sampling of Continuous-Time Signals . . . . . . . 66

4.3.4 The Discrete Fourier Transform(DFT) . . . . . 67

4.3.5 Computation of the Discrete Fourier Transform . . . 6 67

4.3.6 Fourier Analysis of Signals Using the Discrete Fourier Transform . . . . . . . . . . . 68

4.4 Motorola's DSP56000 DSP Family . . . . . . . . . . 70

4.5 Ariel's Interface and DSP Library . . . . . . . . . 74

4.5 .1 Interface Library . . . . . . . . . . . . . . . 74

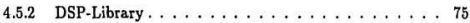

4.6 Application Software for Spectra Collection . . . . . . . 76

4.7 Experimental Setup . . . . . . . . . . . . 76

4.8 Spectra for Different Fault Conditions . . . . . . . . . 78

4.8.1 No Fault condition: . . . . . . . . . . . . . 80

4.8.2 Stator phase 1 is open: . . . . . . . . . . . . 81

4.8.3 Stator phase 2 is open: . . . . . . . . . 82

4.8.4 Stator phase 3 is open: . . . . . . . . . . 82

4.8.5 Short circuit fault through resistance in Stator Phase 1: 83

4.8.6 Short circuit fault through resistance in Stator Phase 2: 84

4.8.7 Short circuit fault through resistance in Stator Phase 3: 85

4.8.8 Short circuit fault in Stator Phase 1: . . . . . 86

4.8.9 Short circuit fault in Stator Phase 2: . . . . . . 87

4.8.10 Short circuit fault in Stator Phase 3: . . . . . . 88

4.8.11 Rotor open circuit fault in phase 1 : . . . . . . . 89

4.8.12 Rotor open circuit fault in phase $2: \ldots \ldots \ldots \ldots$. . 90

4.8.13 Rotor open circuit fault in phase $3: \ldots \ldots \ldots$. . . 91 
4.8.14 Rotor phase 1 is shorted to neural: . . . . . . . . 92

4.8.15 Rotor phase 2 is shorted to neural: . . . . . . . . 93

4.8.16 Rotor phase 3 is shorted to neural: . . . . . . . . 94

4.8.17 Kotor short circuit fault through resistance in phase 1: 95

4.8.18 Rotor short circuit fault through resistance in phase 2: 96

4.8.19 Rotor short circuit fault through resistance in phase 3: 97

4.8.20 Rotor phase 1 is unbalanced through an external resistor: 98

4.8.21 Rotor phase 2 is unbalanced through an external resistor: 99

4.8.22 Rotor phase 3 is unbalanced through an external resistor: 100

4.8.23 Simultancous open circuit fault in Rotor phase 2 as well as Stator phase 1: . . . . . . . . . . . . 101

4.8.24 Simultaneous open circuit fault in Rotor phase 2 as well as Stator phase 2: . . . . . . . . . . . . 102

4.8.25 Simultaneous open circuit fault in Rotor phase 2 as well as Stator phase $3: \ldots \ldots$. . . . . . . . . 103

4.8.26 Remarks on Fault Related Frequenency spectra of the Stator Current ................ 103

5.1 Introduction . . . . . . . . . . . . . . . . . . . . 104

5.2 Training Data Set . . . . . . . . . . . . 105

5.2 .1 Data Reduction: ................ 106

5.3 Structure of the Network . . . . . . . . . . . . . 108

5.4 Training of the Network . . . . . . . . . . . . 109

5.5 Fault Recognition by the trained network. . . . . . . . 111 
5.6 Model of ART2 neural network based Incipient Fault Detection System . . . . . . . . . . . . . 112

A Conclusions and Recommendations for Future Work 116

6.1 Conclusions . . . . . . . . . . . . . . . . 116

6.2 Recommendations for Future Work . . . . . . . . . 118

$\begin{array}{llcc}\text { Appendix-A } & \text { Program listing for ART2 Neural Network. } & \ldots . . . . & 124 \\ \text { Appendix-B } & \text { Program listing for real-time frequency spectra acquisition. } & 141 \\ \text { Appendix-C } & \text { Program listing of } \text { HP-plotter driver. } & \text {........ } & 150\end{array}$ 


\section{List of Figures}

1.1 (a) Frequency spectra of input current of a healthy machine.(b) Frequency spectra of input current of a faulty machine. (c). Model of Neural Network based fault related spectra identification system. . . . . . . . . . . . . 9

2.1 Model of a neuron $\ldots \ldots \ldots \ldots \ldots \ldots \ldots$

2.2 Activation Functions: (a) Threshold Function. (b) PiecewiseLinear Function (c) Sigmoidal Function . . . . . . . . 18

2.3 The three layer BPN architecture . . . . . . . 20

2.4 BAM architecture . . . . . . . . . . . . 22

2.5 Discrete Hopfield Memory . . . . . . . . . . . 25

2.6 A simple energy landscape with two minimal, a local minimum and a global minimum $\ldots \ldots \ldots \ldots \ldots$

2.7 The Botzman Completion Architecture . . . . . . . . 26

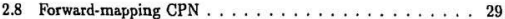

2.9 Layer of input units of a CPN . . . . . . . . . 30

2.10 This figure shows (a) the general form of the processing elements (b) the instar form of processing elements . . . . . 31

2.11 A layer of instars arranged in $\mathrm{c}$ CPN $\ldots \ldots \ldots . \ldots 32$ 
2.12 Outstar and its relationship to the CPN architecture. (a). Outstar structures in CPN network (b). A single outstar unit

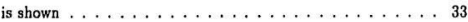

2.13 Grossber's formal avalanche structure . . . . . . . . 36

2.14 Power Spectra generated from speech . . . . . . . . . 37

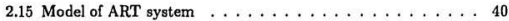

2.16 A pattern matching cycle in an ART. (a) pattern-matching attempt (b) reset in (c) final recognition (d) end of matching cycle . . . . . . . . . . . . . . 4 41

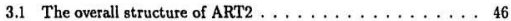

3.2 Structure of processing element on $F_{1}$ layer $\ldots \ldots \ldots 47$

3.3 Structure of processing element on $F_{2}$ layer $\ldots \ldots \ldots$. . 50

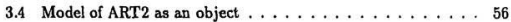

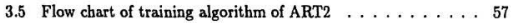

3.6 Modified Training and Recall Algorithm of ART2 . . . . . 58

4.1 Model of Fault Related Spectra Collection . . . . . . . . 64

4.2 Representation of discrete-time system . . . . . . . 65 65

4.3 Processing steps in the discrete-time Fourier analysis of a continuous-

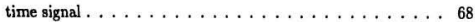


4.4 Illustration of the Fourier transforms in the system : (a) Fourier transform of continuous-time input signal. (b) Frequency response of anti aliasing filter. (c) Fourier transform output of anti aliasing filter. (d) Fourier transform of sampled signal (e) Fourier transform of window sequence (f) Fourier transform of windowed signal segment and frequency samples obtained using DFT samples . . . . . . . . . . . . . 69

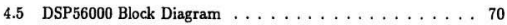

4.6 PC-56 Block Diagram . . . . . . . . . . . . . 74

4.7 Flowchart of the application program . . . . . . 77

4.8 Block diagram of the experimental setup. . . . . . . . 78

4.9 Frequency spectra at no fault condition $\ldots \ldots \ldots . \ldots 80$

4.10 Frequency spectra when stator phase 1 is open . . . . . . 81

4.11 Frequency spectra when stator phase 2 is open . . . . . 82

4.12 Frequency spectra when stator one coil of phase 1 has been externally replaced by a resistor $\ldots \ldots \ldots \ldots$

4.13 Frequency spectra when stator one coil of phase 2 has been externally replaced by a resistor . . . . . . . . . 84

4.14 Frequency spectra when stator one coil of phase 3 has been externally replaced by a resistor . . . . . . 85

4.15 Frequency spectra when stator one coil of phase 1 was short . 86 
4.16 Frequency spectra when stator one coil of phase 2 was short . 87

4.17 Frequency spectra when stator one coil of phase 3 was short . 88

4.18 Frequency spectra of the stator current when rotor one coil M1 was open circuit . . . . . . . . . . . . . . 89

4.19 Frequency spectra of the stator current when rotor one coil M2 was open circui .................... 90

4.20 Frequency spectra of the stator current when rotor one coil M3 was open circui . . . . . . . . . . . . . . . . . 91

4.21 Frequency spectra of the stator current when rotor phase M1 is shorted to neutral . . . . . . . . . . . . . 92

4.22 Frequency spectra of the stator current when rotor phase M2 is shorted to neutral $\ldots \ldots \ldots$

4.23 Frequency spectra of the stator current when rotor phase M3 is shorted to neutral . . . . . . . . . . . . . . 94

4.24 Frequency spectra of the stator current at rotor short circuit fault through resistance in phase M1 ..........95

4.25 Frequency spectra of the stator current at rotor short circuit fault through resistance in phase M2 ......... 96

4.26 Frequency spectra of the stator current at rotor short circuit fault through resistance in phase M3 . . . . . . . 97

4.27 Rotor phase 1 is unbalanched through an external resistance . 98

4.28 Rotor phase 2 is unbalanched through an external resistance . 99

4.29 Rotor phase 3 is unbalanched through an external resistance. 190

4.30 Simultaneous open circuit fault in rotor phase 2 as well as stator phase $1 \ldots \ldots \ldots \ldots 1 . \ldots \ldots 1$ 
4.31 Simultaneous open circuit fault in rotor phase 2 as well as stator phase2 . . . . . . . . . . . . 102

5.1 Model of ART2 neural network based on-line incipient fault diagnosis system for induction motors. . . . . . . 115 


\section{List of Tables}

2.1 Comparative Performance of Different ANN Techniques ... . 44

3.1 Values of parameters on F-1 layer . . . . . . . 48

3.2 Set of training examples used to train ART2 . . . . . 59

3.3 Bottom-up weight matrix after training . . . . . . 59

3.4 Top-down weight matrix after training $\ldots \ldots \ldots \ldots \ldots 59$

3.5 New training vector to train after neuron addition . . . . 60

3.6 New Bottom-up weight matrix after training $\ldots \ldots \ldots 60$

3.7 New Top-down weight matrix after training . . . . . . 61

3.8 Pattern matching result of the trained network $\ldots \ldots \ldots 62$

5.1 Table of Faults with unique number. . . . . . . . 105

5.2 Fault related spectral components in matrix form . . . . 106

5.3 Training Matrix of fault related current spectra. . . . . . 107

5.4 Faults mapping of the trained network in high precision domain 108

5.5 Top-down weight matrix of the trained network. . . . . 110

5.6 Bottom-up weight matrix of the trained network. . . . . . 110

5.7 Fault related noisy current spectra. . . . . . . . . 113

5.8 Diagnostic test result of the trained network in noisy situation. 113

5.9 Faults mapping in training phase in low precision domain. . . 114

5.10 Fault diagnostic performance of the network trained in low precision domain in noisy situation . . . . . . . . 114 


\section{Abbreviations and Symbols:}

The following lists of terms and symbols appear throughout the body of this document. They are defined here approximately in the order in which they appear in the text:

Abbreviations:

\begin{tabular}{|l|l|}
\hline \multicolumn{1}{|c|}{ Terms } & \multicolumn{1}{|c|}{ Definition } \\
\hline \hline ANN & Artificial neural network \\
\hline FNN & Feedforward neural network \\
\hline DSP & Digital signal processing \\
\hline BPN & Backpropagation neural network \\
\hline BAM & Binarj' associative mernory \\
\hline HM & Hpfield memory \\
\hline VLSI & Very large scale integration \\
\hline CPN & Counter propagation network \\
\hline SOM & Self-organizing maps \\
\hline STNS & Spatiotemporal networks \\
\hline ART & Adaptive resorance theory \\
\hline STM & Short term memory \\
\hline LTM & Long term memory \\
\hline DFT & Discrete Fourier transform \\
\hline FFT & Fast Fourier transform \\
\hline RAM & Random access memory \\
\hline ROM & Read only memory \\
\hline ALU & Arithmetic logic unit \\
\hline AGU & Address generation unit \\
\hline MCU & Microcontroller unit \\
\hline MPU & Microprocessor unit \\
\hline MIP & Million instructions per second \\
\hline IIR & Infinite impulse response \\
\hline & \\
\hline
\end{tabular}


Abbreviations:

\begin{tabular}{|l|l|}
\hline \multicolumn{1}{|c|}{ Terms } & \multicolumn{1}{c|}{ Definition } \\
\hline \hline SCI & Serial communication interface \\
\hline SSI & Synchronous serial interface \\
\hline PC & Personal computer \\
\hline ARIELMON & Ariel monitor \\
\hline DEGMON & Debugg monitor \\
\hline IBM & Internation Buisiness Machine \\
\hline HP & Hewlet \& Packard \\
\hline AS & Attentional subsystem \\
\hline PE & Processing element \\
\hline
\end{tabular}

Symbols:

\begin{tabular}{|l|l|}
\hline \hline Symbols & \multicolumn{1}{|c|}{ Definition } \\
\hline \hline$i_{s}$ & Stator current \\
\hline$\phi_{\mathrm{r}}$ & Rotor flux \\
\hline$v_{s}$ & Stator voltage per phase \\
\hline$R_{s}$ & Stator resistance per phase \\
\hline$R_{r}$ & Rotor resistance per phase \\
\hline$L_{s}$ & Stator self inductance per phase \\
\hline$L_{r}$ & Rotor self inductance per phase \\
\hline$M$ & Mutual inductance \\
\hline$\delta$ & Leakage coefficient \\
\hline$\omega_{r}$ & Angular velocity \\
\hline$u_{k}$ & Linear combiner output \\
\hline$w_{k j}$ & Synaptic weight of synapse j belonging to neuron $\mathrm{k}$ \\
\hline
\end{tabular}




\section{Symbols:}

\begin{tabular}{|c|c|}
\hline Symbols & Definition \\
\hline$x_{j}$ & Input signal $\mathrm{j}$. \\
\hline$y_{k}$ & the output signal of neuron $k$ \\
\hline$\varphi$ & The activation function \\
\hline$\theta_{k}$ & The threshold \\
\hline$v_{k}$ & The activity level \\
\hline $\bar{x}$ & Input vector \\
\hline net $_{p j}^{h}$ & Net input values to the hidde? layer units \\
\hline$O_{p k}$ & Calculated output \\
\hline$E_{p}$ & Measure of learning \\
\hline$w$ & Weight vector \\
\hline E & System energy \\
\hline$\Delta E_{k}$ & The energy difference \\
\hline$\theta_{i}$ & The reflectance pattern \\
\hline$x[n]$ & Discrete input sequence \\
\hline T. & Discrete operator \\
\hline$y[n]$ & Discrete output sequence \\
\hline$S_{c}(t)$ & Band-unlimited continuous-time signal \\
\hline$x_{c}(t)$ & Bandlimited continuous-time signal \\
\hline$X[k]$ & Discrete fourier transform \\
\hline$\Omega_{n}$ & Nyquist sampling frequency \\
\hline$J_{k}^{+}$ & Excitory input \\
\hline$J_{k}^{-}$ & Inhibitory input \\
\hline $\mathrm{z}$ & Weight vector in ART network \\
\hline$M$ & Number of units in input of ART network \\
\hline$N$ & Number of output units of ART network \\
\hline
\end{tabular}




\section{Chapter 1}

\section{Introduction}

Fault diagnosis has been an active area of research in both the engineering and computer science communities. In spite of the many advances in this area, fault diagnosis still remains challenging with many questions unanswered. With advances made in technology, complexity is a major factor that we have to deal with. Complexity confronts us, perhaps, when something breaks down. We are forced to come up with more effective techniques and analysis for detecting and diagnosing such anomalies. Diagnostic analysis is not always quantitative. In fact much detection and diagnostic analysis is heuristic, and results from repeated and long time cognitive experiences. In the last decades, ac drive installation has shown tremendous growth both in size and complexity. The interruption of service due to faults in a motor drive installation is often costly and could interfere with public safety in some installations. 
These motors are exposed to a wide variety of environments and conditions which make the motor subject to incipient faults. These incipient faults, if left undetected, contribute to the degradation and eventual failure of the motors. With proper monitoring and fault detection schemes, the incipient faults can be detected in their early stages, and maintenance and down time expenses can be reduced while also improving safety. Thus, the motor incipient fault detection can be used in the motor preventive maintenance programs also.

\subsection{Incipient Fault Detection of Induction Motors}

\subsubsection{A Brief Description}

Although rotating machines are usually well constructed and robust, the possibility of incipient faults is inherent in the machines due to the stresses involved in the conversion of electrical energy to mechanical energy and vice versa [1, 2]. Incipient faults within a machine will affect the performance of the machine before major failures occur. With proper system monitoring and fault detection schemes, maintenance costs can be reduced and reliability of the machines can be improved significantly.

An experienced engineer may detect and diagnose the motor faults by observing the motor's operating performances. However, experienced engineers are expensive and difficult to train. It is, therefore desirable to automate 
the system monitoring and fault detection schemes rather than to rely on an expert to perform continuous on-line monitoring. Several fault detection methods have been developed, each with their own prospects and constraints. Some techniques require expensive diagnostic equipment and/or off-line fault analysis to determine the motor condition. For instance, the radio frequency monitoring scheme injects radio frequency signals to the stator winding of a machine and measures the changes of the signal waveform to determine whether the winding insulation contains faults [2]. This technique requires expensive equipment and is justified only for use with large and expensive machines. Other popular techniques, such as particle analysis which requires bringing the motor oil samples to a laboratory for analysis [2] to determine the motor condition, are more suitable for overhaul or routine check-up rather than on-line monitoring and fault detection.

The parameter estimation approach [3] is a non-invasive fault detection scheme. Non-invasive fault detection schemes are based on easily accessible and inexpensive measurements to predict the motor condition without disintegrating the motor structure. These schemes are suitable for on-line monitoring and fault detection purposes. Due to their economical and nondestructive features, non-invasive techniques are often preferred by many engineers. However, the parameter estimation approach requires an accurate mathematical model and an elaborate understanding of the system dynamics based on a set of system parameters. The parameters arc usually chosen to reflect the motor conditions. For example, the bearing condition will affect the damping coefficient of the motor's mechanical equation. As the 
bearing wears out, the damping coefficient increases. Thus, the parameter estimation approach can be based on the motor's mechanical equation and measurements to estimate the value of the damping coefficients. After estimating the numerical value of the chosen parameter, a means to translate the estimated numerical values to qualitative description is required. The major difficulty with the parameter estimation approach is that an accurate mathematical model is required, and is usually difficult to obtain. Other techniques like non-parametric surface fitting method also require case by case specific mathematical analysis. In addition, the interpretation of the fault conditions, which is a fuzzy concept using rigorous mathematical formulations, is generally impractical and inaccurate.

On the other hand, use of an artificial neural network for fault detection is also a non-invasive technique $[3,4]$. But, unlike the parameter estimation approach, neural networks cen perform fault detection based on measurements and training without the need of complex and rigorous mathematical models. In addition, heuristic interpretation of the motor conditions, which sometimes only humans are capable of doing, can be easily implemented in the neural network through supervised training.

\subsubsection{Mathematical Analysis of Induction Motor}

In order to successfully perform fault detection, different sets of criteria are needed to define a motor's status at different operating conditions. The fault detection of a 3-phase induction motor has been used for illustration 
purposes. It is worthwhile to describe the fault detection problem in mathematical terms to facilitate future discussions on the subject.

\section{Mathematical Description of Motor Dynamics}

An induction motor can be described by the following state equations in the siaticnary reference frame:

$$
\begin{gathered}
\frac{d}{d t}\left[\begin{array}{l}
i_{1} \\
\phi_{r}
\end{array}\right]=\left[\begin{array}{ll}
A_{11} & A_{12} \\
A_{21} & A_{22}
\end{array}\right]\left[\begin{array}{l}
i_{1} \\
\phi_{r}
\end{array}\right]+\left[\begin{array}{c}
B_{1} \\
0
\end{array}\right] v_{s} \\
=A x+B v, \\
i_{s}=C x
\end{gathered}
$$

Where

$$
\text { Staior Current } i_{s}=\left[i_{d s} i_{q s}\right]^{T}
$$

•

$$
\text { RotorFlux } \phi_{r}=\left[\phi_{d r} \phi_{\text {or }}\right]^{T}
$$

$$
\begin{aligned}
& \text { Stator Voltage } v_{s}=\left[v_{d a} v_{q s}\right]^{T} \\
& \text { - } A_{11}=-\left(R_{a} /\left(\delta L_{a}\right)+(1-\delta) /\left(\delta \tau_{r}\right)\right) \mathrm{I}=a_{r 11} \mathrm{I} \\
& \text { - } A_{12}=M /\left(\delta L_{a} L_{r}\right)\left(\left(1 / \tau_{r}\right) \mathrm{I} \omega_{r} \mathrm{~J}\right)=a_{r 12} \mathrm{I}+a_{i 12} \mathrm{~J}
\end{aligned}
$$


- $A_{21}=\left(M / \tau_{r}\right) \mathrm{I}=a_{r 21} \mathrm{I}$

- $A_{22}=-\left(1 / \tau_{r}\right) \mathrm{I}=\omega_{r} \mathrm{~J}=a_{r 22} \mathrm{I}+a_{i 22} \mathrm{~J}$

- $B_{1}=1 /(\delta L,) \mathrm{I}=b_{1} \mathrm{I}$

$$
\begin{aligned}
& C=\left[\begin{array}{ll}
I & 0
\end{array}\right] \\
& I=\left[\begin{array}{ll}
1 & 0 \\
0 & 1
\end{array}\right] \\
& J=\left[\begin{array}{cc}
0 & -1 \\
1 & 0
\end{array}\right]
\end{aligned}
$$

$R_{g}$ and $R_{r}$ are stator and rotor resistances, respectively, $L_{g}$ and $L_{r}$ are stator and rotor self inductances, respectively, $M$ is mutual inductance, Leakage coefficient $\delta=1-M^{2} /(L, L-r)$,

Rotor time constant $\tau_{\mathrm{r}}=L-r / R_{r}$ and $\omega_{\mathrm{r}}$ is motor angular velocity in radian/seconds.

Input current depends on the motor parameters $R_{n}, R_{r}, L_{n}, L_{r}, M$ and $\delta$. An internal fault in the machine will be reflected in the stator current of the machine. It is possible therefore, to detect the fault from the analysis of stator current. 


\subsection{Artificial Neural Network for Fault De- tection}

\subsubsection{Expert Approach for Fault Detection}

As stated previously, the interpretation of a motor's condition based on numerical value is usually a difficult task because fault detection is a fuzzy concept and usually requires experience [5]. Therefore, in many cases, heuristic interpretation of the results, which only humans are capable of doing becomes necessary. An experienced engineer can diagonose the motor's condition based on its operating conditions and measurements without knowing exact mathematival model of the motor. The approach is simple and reliable, and the complicated mathematical relation is embedded in the engineer's knowledge about the motor. However, an experienced engineer may not be able to give detailed explanations regarding his/her reasoning and logic used to make the decisions, simply because experience belongs to the fuzzy logic realm and is difficult to describe accurately in exact mathematical terms.

As it turns out, this human expertise approach has many advantages over the parameter estimation approach. However, the major drawback of the human expertise approach is that experience is difficult to transfer and automate. Both researchers and engineers usually transfer experience and knowledge through languages and mathematics, which are sometimes time consuming and inaccurate. In practice, the experience and the knowledge used by expert engineers to perform motor fault detection and or diagnosis 
historical fault detection data gathered by the experts.

\subsubsection{Learning Skills of Artificial Neural Networks}

With the emerging technology of artificial neural networks, the human expertise approach can be mimicked and automated $[6,7,8]$. Artificial neural networks(ANN) can be trained to perform motor fault detection by learning expert's knowledge using a representative set of motor data [9]. In the case of an induction motor, incipient faults can be detected by analyzing the frequency spectrum of the stator current as shown in Fig. 1.1(a)-(b). Now it is clear that the stator current spectrum carries the signature of an internal fault within the machine. So by training an ANN with the values of spectral component related to particular faults without the need of mathe. matical models, the complexity of the parameter estimation approach can be avoided. Once the ANN is trained appropriately, the network weights contain the knowledge needed to perform fault detection, which is equivalent to the expertise gained by an engineer over the years in machine fault diagnosis.

\subsection{Literature Review}

ANNs have been proven to be capable of successfully performing motor fault detections $[9,10]$. One of the advantages of this type of pattern recognition techniques is that it can save time in information processing in run time, where all the computational complexities are done off-line in the training 


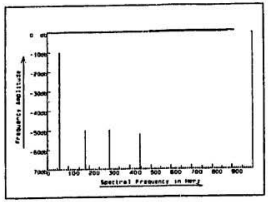

(a)

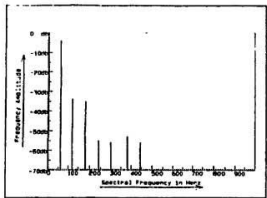

(b)

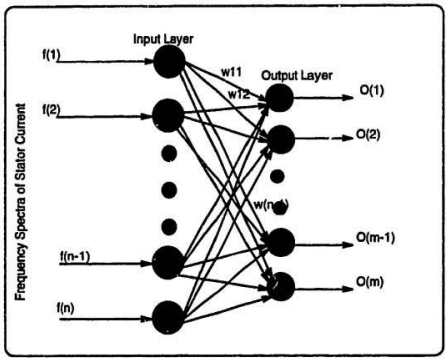

(c)

Figure 1.1: (a) Frequency spectra of input current of a healthy machine.(b) Frequency spectra of input current of a faulty machine. (c). Model of Neural Network based fault related spectra identification system. 
period of the network.

When developing an ANN based system to perform a particular pattern classification problem, typically the process is to gather a set of examples or training patterns, then using these examples to train the underlying ANN. During the training, the information is coded in the system by the adjustments of the weight values. Once the training is deemed to be adequate, the system is ready to be used in the real-time situations, and usually no additional weight modification is required.

This operational scenario is acceptable provided the problem domain has well-defined boundaries and is stable. Under such conditions it is possible to define an adequate set of training inputs for whatever problem being solved. Unfortunately, like many realistic situations involving incipient fault detection of induction motors, the environment is neither bounded nor stable. To solve this dynamic behaviour conventional Feed-forward Neural Network(FNN) suffers a major set back.

Mo-Yuen Chow and others [3]-[5],[9]-[11] have done significant works in neural network based incipient fault diagnosis of induction motors. But they have used FNN as fault diagnosis tool and in their research work they have neglected this dynamic operational scenario which is an indispensable part in real-world environment. M.F. Abdel Mageed and his colleagues [12] have used Hierarchical neural network, but this neural network also suffers the same limitation as FNN. The same limitation also prevails in the research works of F.Filippetti [13, 14], Chin-Teng [6] and their colleagues. Moreover, 
the reporting ability of the neural network, if it cannot diagnose a particilar fault has not been considered by them. So, there is a need to carry out a research work to find suitable neural network which is not only capable to diagonose a fault but also can report if it cannot, so that preventive steps can be taken to update the neural network to adapt to this new fault, while retaining the already acquired knowledge without retraining of the already trained patterns.

Mathematical analysis as mentioned in section(1.1.2) makes sense that wave shape of the stator current carries the signature of internal condition of the machine. R. Natarajan [15] has used the stator current to diagonise the fault by only measuring its value, not though the spectral analysis, which is necessary for neural network based fault detection scheme to get better result. F.Filippetti and M.Martelli [13] have considered the frequency spectra of the stator current as the key fault related information carrier of the fault diagnosis scheme, but they have not reported a detailed study of frequency spectra of the stator current at different fault conditions. B.C. Papadias [16] and others have given an outline to develop an expert system for troubleshooting of electrical machines, but the collection of fault related information is not mentioned. While the focus of research work of Mo-Yuen Chow and others [3]-[5],[9]-[11] is towards the applicability of neural network in the incipient fault diagnosis of induction motors specially in bearing fault, they have not considered the spectral analysis of the stator current. At this present state, it is evident that it is important to pay attention to collect the frequency spectra of the stator current at different fault conditions. 


\subsection{Objective of the Present Work}

The long-term objective of this work is to improve the state-of-the-art of incipient fault diagnosis of induction motors. The specific short-term objectives are summarized in the following three points:

- To find a neural network suitable for incipient faults detection of electric machines. This fault diagnosis scheme is not only capable of detecting a fault but also can report if it cannot identify a particular fault so that necessary preventive steps can be taken to update the underlying neural network to adapt to this undetected fault.

- To develop a laboratory set-up to collect frequency spectra of the stator current of induction motor in real-time using digital signal processing techniques, and to collect frequency spectra of the stator current at different fault conditions for the experimental induction motor.

- To train the selected neural network with fault related frequency spectra of the stator current and to study the performance of the trained network to diagonise faults in noise free as well as noisy conditions.

\subsection{Overview of Thesis}

The contents of the theses can be summarized in the following chapters: 
Chapter 2 covers a survey of available neural networks. A comparative study has been done to select a neural network to satisfy one of the objectives.

Chapter 3 discusses the software implementation of the selected neural network. It's performance has been tested with a number of examples.

Chapter 4 explains the related algorithms and techniques to collect the fault related frequency spectra of the stator current of a three phase induction motor. This chapter also gives a brief outline of the digital signal processing (DSP) board as well as the DSP library. Salient features of the software developed as part of this work to acquire real time frequency spectra of the stator current has been also described in this chapter.

Chapter 5 explains the performances of the selected neural network to classify faults based on fault related frequency spectra of the stator current. A model baseci on the selected neural network for on-line incipient fault diagnosis system for the induction motor has also been reported in this chapter.

Chapter 6 contains the conclusions and recommendations for future work. It has been explained that there is a good prospect to do further work on the irrespective of design parameter, type of machine as well as operating conditions fault diagnosis system. Moreover, the scope of development of multi-machine fault diagnosis system to make it costeffective and user-friendly has also been emphasized. 


\section{Chapter 2}

\section{An overview of Artificial Neural Networks}

\subsection{Introduction}

A neural network is a massively parallel distributed processo" that has natural propensity for storing experimental knowledge and making it available for use [17]. It mimics the brain in two respects:

a). Knowledge is acquired by the network through a learning process.

b). Inter-neuron connection strengths, usually known as synaptic weights, are used to store the knowledge.

The procedure used to perform the learning process is called a "learning algorithm", the function of which is to modify the synaptic weights of the network in an orderly fashion so as to attain a desired design objective. The 


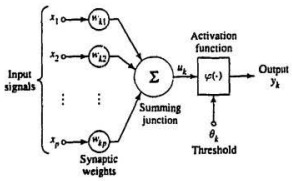

Figure 2.1: Model of a neuron

use of neural networks offers a number of benefits, among them nonlinearity, input-output mapping and adaptivity are most important.

\subsubsection{Models of a Neuron}

Fig. 2.1 shows the model of a neuron. Three basic elements of the neuron are explained as follows:

1. A set of synapses or connecting links, each of which is characterized by a weight or strength of its own.

2. An Adder for summing the input signals.

3. An activation function for limiting the amplitude of the output of the neuron. 
A neuron $K$ can be explained by the following equations:

$$
\begin{aligned}
u_{k} & =\sum_{j=1}^{p} w_{k j} x_{j} \\
y_{k} & =\varphi\left(u_{k}-\theta_{k}\right)
\end{aligned}
$$

where $x_{1}, x_{2}, \ldots, x_{p}$ are input signals; $w_{k 1}, w_{k 2}, \ldots, w_{k p}$ are the synaptic weights of neuron $k ; u_{k}$ is the linear combined output; $\theta_{k}$ is the threshold; $\varphi$ is the: activation function; and $y_{k}$ is the output signal of the neuron. The use of threshold $\theta_{k}$ has the effect of applying an affine transformation to the output $u_{k}$ of the linear combiner in the model as shown by

$$
v_{k}=u_{k}-\theta_{k}
$$

The output can be represented by the following equation:

$$
y_{k}=\varphi\left(v_{k}\right)
$$

Types of activation function

Generally used three different types of activation functions are described below [17]:

\section{Threshold Function:}

For this type of function as shown in Fig. 2.2(a)

$$
\begin{aligned}
& \varphi(v)= \begin{cases}1 & \text { if } v \geq 0 \\
0 & \text { if } v<0\end{cases} \\
& y_{k}= \begin{cases}1 \text { if } v_{k} \geq 0 \\
0 \text { if } v_{k}<0\end{cases}
\end{aligned}
$$




$$
v_{k}=\sum_{j=1}^{p} w_{k j} x_{j}-\theta_{k}
$$

\section{Piecewise-Linear Function:}

A Piecewise-linear function as shown in Fig. 2.2(b) can be explained by the following equation:

$$
\varphi(v)= \begin{cases}1, & \text { if } v \geq \frac{1}{2} \\ v, & \text { if }-\frac{1}{2}>v>-\frac{1}{2} \\ 0, & \text { if } v \leq-\frac{1}{2}\end{cases}
$$

\section{Sigmoidal Function:}

The Sigmoidal function as shown in Fig. 2.2(c) is by far the most common form of activation function used in the construction of artificial neural networks as explained by the following equation:

$$
\varphi(v)=\frac{1}{1+\exp (-a v)}
$$

where $a$ is the slope parameter.

\subsubsection{Network Architecture}

Learning algorithm to train a neural network depends on the way it is structured. In general, there are four different classes of network architectures:

1. Single-Layer Feedforward Networks.

2. Multilayer Feedforward Network.

3. Recurrent Networks.

4. Lattice Structures 

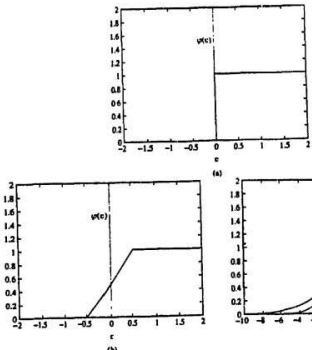

(1)

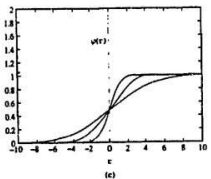

Figure 2.2: Activation Functions: (a) Threshold Function. (b) PiecewiseLinear Function (c) Sigmoidal Function

\subsubsection{Artificial Intelligence and Neural Networks}

The aim of artificial intelligence(AI) is the development of paradigms or algorithms that require machines to perform tasks that apparently require cognition when performed by humans. An AI system must be capable of:

1. storeing knowledge;

2. appling the knowledge stored to solve problems;

3. acquiring new knowledge through experience; 
An Al system has three key components: representation, reasoning, and learning. AI can be described as the formal manipulation of a language of algorithms and data representations in a top-down fashion. On the other hand, neural network can be described as parallel distributed processors with a natural learning capability, and which usually operate in bottom-up fashion. For the implementation of cognitive tasks, it therefore appears that rather than seek solution based on $\mathrm{AI}$ or neural network alone, a more potentially useful approach would be to build structured connectionist models that incorporate both of them.

Some important neural networks have been explained briefly in the folowing sections ending with a comparative analysis for the selection of proper neural network for incipient fault detection of an induction motor.

\subsection{Backpropagation}

Backpropagation neural network(BPN) as shown in Fig. 2.3 learns a predefined set of input-output example pairs by using a two-phase propagate-adapt cycle [18]. After an input pattern has been applied as stimulus to the first layer of the network units, it is propagated through each upper layer until an output is generated. This output pattern is then compared to the desired output,and error signal is computed for each output unit. The error signals are then transmitted backward from the output layer to each node in the intermediate layer that contributes directly to the output. However, each node in the intermediate layer receives only a portion of the total er-

ror signal, based roughly on the relative contribution the unit made to the 


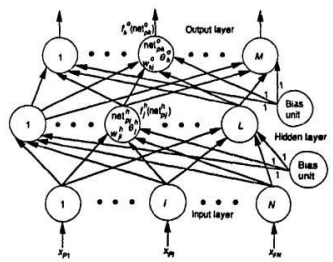

Figure 2.3: The three layer BPN architecture

original output. This process repeats, layer by layer until each node in the network has received an error signal that describes its relative contribution to the total error. The training procedure of a BPN can summarized in the following points:

1. The vector, $\mathbf{x}_{p}=\left(x_{p 1}, x_{p 2}, \ldots \ldots, x_{p n}\right)^{t}$ is applied to the input units.

2. The net-input values to the hidden layer units are calculated:

$$
n e t_{p j}^{h}=\sum_{i=1}^{N} w_{j i}^{h} x_{p i}+\theta_{j}^{h}
$$

3. The outputs from the hidden layer are calculated:

$$
i_{p j}=f_{j}^{h}\left(n e t_{p j}^{h}\right)
$$

4. For output layer the net-input values to each unit is calculated:

$$
n e t_{p k}^{\circ}=\sum_{j=1}^{L} w_{k j}^{o} i_{p j}+\theta_{k}^{o}
$$


5. The outputs are calculated:

$$
O_{p k}=f_{k}^{\circ}\left(n e t_{p k}^{\circ}\right)
$$

6. The error terms of the output units are calculated:

$$
\delta_{p j}^{h}=f_{j}^{h^{\prime}}\left(n e t_{p j}^{h}\right) \sum_{k} \delta_{p k}^{\circ} w_{k j}^{0}
$$

7. The weight on the output layer is updated:

$$
w_{k j}^{o}(t+1)=w_{k j}^{o}(t)+\eta \delta_{p k}^{o} i_{p j}
$$

8. Weights in the hidden layer are updated:

$$
w_{j i}^{h}(t+1)=w_{j i}^{h}(t)+\eta \delta_{p j}^{h} x_{i}
$$

The following equation is the measure of how well the network is learning.

$$
E_{p}=\frac{1}{2} \sum_{k=1}^{M} \delta_{p k}^{2}
$$

When the error is acceptably small for each of the training-vector pairs, training can be discontinued.

\subsection{The Binary Associative Memory(BAM) and the Hopfield Memory}

A type of memory called an associative memory is a subject matter of this section. In fact, the concept of associative memory is a fairly intuitive one: associative memory appears to be one of the primary functions of the brain [18]. 


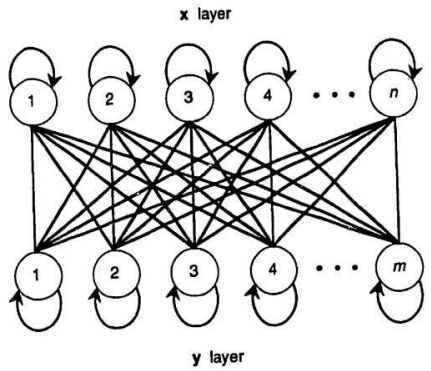

Figure 2.4: BAM architecture

\subsubsection{The BAM}

The BAM consists of two layers of processing elements that are fully interconnected between the layers. The units may, or may not, have feedback connection to themselves. The general case is shown in Fig. 2.4. For the $L$ vector pairs that constitute the set of examplers should be stored, the following matrix can be constructed:

$$
\mathbf{w}=\mathrm{y}_{1} \mathbf{x}_{1}^{t}+\mathrm{y}_{2} \mathrm{x}_{2}^{t}+\ldots \ldots+\mathrm{y}_{L} \mathbf{x}_{L}^{t}
$$

This equation gives the weights on the connections from the $\mathbf{x}$ layer to the $\mathbf{y}$ layer. To construct the weights for the $x$ layer units, it is necessary simply to take the transpose of the weight matrix, $w^{t}$. 


\section{BAM Mathematics}

On the $y$ layer

$$
\text { net }^{y}=\mathbf{w x}
$$

where net ${ }^{y}$ is the vector of the net-input values on the $y$ layer. In terms of the individual units, $y_{i}$,

$$
n e t_{i}^{y}=\sum_{j=1}^{n} w_{i j} x^{j}
$$

On the $\mathrm{x}$ layer

$$
\begin{gathered}
\text { net }^{x}=\mathbf{w}^{t} \mathbf{y} \\
\text { net }_{i}^{x}=\sum_{j=1}^{m} y_{j} w_{j i}
\end{gathered}
$$

The quantities $n$ and $m$ are dimensions of the $x$ and $y$ layers, respectively.

The output values for each processing element depends on the net input value, and on the current output value of the layer. The new value of $y$ at time step $t+1, y(t+1)$ is related to the value of $y$ at time step $t, y(t)$ by

$$
y_{i}(t+1)= \begin{cases}+1, & n e t_{i}^{y}>0 \\ y_{i}(t), & n e t_{i}^{y}=0 \\ -1, & n e t_{i}^{y}<0\end{cases}
$$

Similarly, $x(t+1)$ is related to $x(t)$ by

$$
x_{i}(t+1)= \begin{cases}+1, & n e t_{i}^{x}>0 \\ x_{i}(t), & n e t_{i}^{x}=0 \\ -1, & n e t_{i}^{x}<0\end{cases}
$$

\section{BAM Processing}

To recall the information using the BAM, the following steps should be performed: 
1. The initial vector pair, $\left(\mathbf{x}_{0}, \mathbf{y}_{\mathbf{0}}\right)$ is applied to the input elements of the BAM.

2. Information is propagated from the $x$ layer to the $y$ layer; and the values on the $y$-layer are updated.

3. The updated $\mathbf{y}$ information is propagated back to the $\mathbf{x}$ layer and the units are updated.

4. Steps 2 and 3 are repeated until there is no further change in the units on each layer.

\subsubsection{The Hopfield Memory}

Hopfield memory(HM) can described as a derivative of the BAM [18]. There are two types of Hopfield memory as described below.

\section{Discrete Hopfield Memory}

Fig. 2.5 illustrate the structure of discrete Hopfield Memory.

\section{Continuous Hopfield Memory}

Continuous Hopfield Memory has same useful properties of associative memory but it can accept analog input making it closer to natural neuron. Moreover, it can be represented by analog eletronic circuit making it suitable for VLSI implementation. 


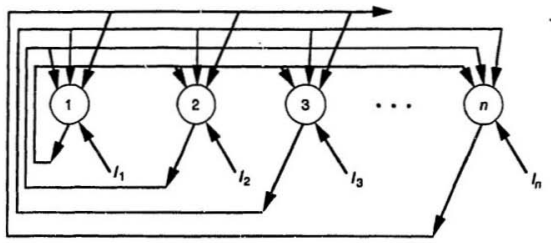

Figure 2.5: Discrete Hopfield Memory

\subsection{Simulated Annealing}

It is possible to extend the analogy between information theory and statistical mechanics in order to place neural network [18] in contact with a heat reservoir at some, as yet undefined temperature. If so, then it is possible to perform a simulated annealing process whereby gradually lowering the temperature while processing takes place in the network, in the hopes of avoiding a local minimum on the energy landscape as shown in Fig. 2.6. This situation can be better explained in the neural network known as Boltzman Machine.

\subsubsection{The Boltzman Machine}

The basic architecture of this type of neural network can be explained by Fig. 2.7 [18]. 


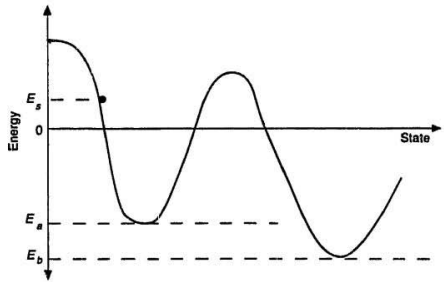

Figure 2.6: A simpie energy landscape with two minimal, a local minimum and a global minimim

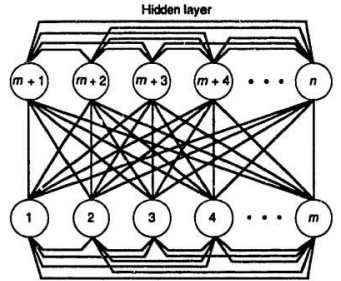

Visible layer

Figure 2.7: The Botzman Completion Architecture 
As the discrete HM, the system energy can be calculated from

$$
E=-\frac{1}{2} \sum_{i=1}^{n} \sum_{j=1, y i} w_{i j} x_{i} x_{j} .
$$

Where $n$ is the total number of units in the network, and $x_{k}$ is the output of the kth unit. The energy difference between the system with $x+k=0$ and $x_{k}=1$ is given by

$$
\Delta E_{k}=\text { net }_{k}
$$

The recall procedure is done by the simulated annealing procedure with $\mathrm{x}^{\prime}$ as the starting vector on the visible units. The procedure is described by the following algorithm:

1. All the outputs of all known visible units are forced to the values specified by the initial input vector, $\mathbf{x}^{\prime}$

2. All unknown visible units and all hidden units are assigned random output values from set 1,0 .

3. A unit, $x_{k}$, at random is selected and its net-input, net ${ }_{k}$ is calculated.

4. Steps 3 and 4 are repeated until all units have had some probability of being selected for update. This number of unit-updates defines a processing cycle.

5. Step 5 is repeated for several processing cycle, until thermal equilibrium has been reached at given temperature, $T$.

6. Temperature $T$ is lowered and step 3 through 7 are repeated. 


\section{Learning in Boltzman Mechines}

A reasonable approach to train a Boltzman machine can summarized in the following way:

1. Artificially the temperature of the Boltzman machine is raised to some finite value.

2. The system is annealed until the equilibrium is reached at some low temperature.

3. The weights of the network is adjusted so that the difference between the observed probability distribution and canonical disiribution is reduced.

4. Steps 1 through 3 are repeated until the weights no longer change.

\subsection{The Counter Propagation Network}

For a given set of vector pairs, $\left(x_{1}, y_{1}\right),\left(x_{2}, y_{2}\right), \ldots \ldots,\left(x_{n}, y_{n}\right)$, the counter propagation network(CPN) can learn to associate an vector $\mathbf{x}$ on the input layer with a vector $y$ at the output layer [18]. If the relationship between $x$ and $y$ can be described by a continuous function $\phi$, such that $y=\phi(x)$, then CPN will learn to approximate this mapping for any value of $x$ in the range specified by the set of training vectors. This situation is known as forward mapping of CPN and its structure is shown in Fig. 2.8. 


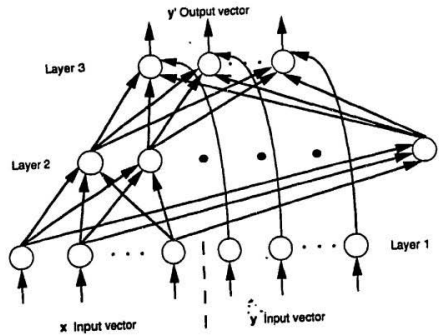

Figure 2.8: Forward-mapping CPN

\subsubsection{CPN Building Blocks}

The building blocks of CPN are explained in following section:

\section{The Input Layer}

The input layer of processing elements is shown in Fig. 2.9. The total input pattern intensity is given by $I=\sum_{i} I_{i}$. Corresponding to each $I_{i}$, a quantity can be defined

$$
\theta_{i}=I_{i}\left(\sum_{i} I_{i}\right)^{-1}
$$

The vector, $\left(\Theta_{1}, \Theta_{2}, \ldots \ldots . . ., \Theta_{n}\right)^{t}$ is called a reflectance pattern. It should be noted that this pattern is normalized in the sense that $\sum_{i} \theta_{i}=1$. 


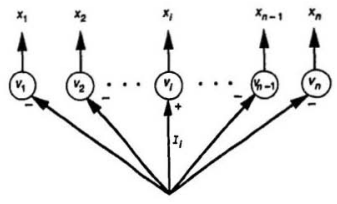

Figure 2.9: Layer of input units of a CPN

\section{The Instar}

The instar is a single processing element as shown in Fig. 2.10

Assuming the initial output is zero, and that a nonzero input vector is present from time $t=0$ until time, $t$ when the output can be defined

$$
y(t)=\frac{b}{a} n e t\left(1-e^{-\mathrm{at}}\right)
$$

The equilibrium value of $y(t)$ is defined by

$$
y^{e q}=\frac{b}{a} \text { net. }
$$




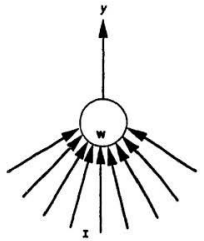

(a)

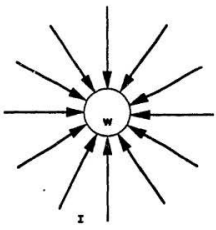

(b)

Figure 2.10: This figure shows (a) the general form of the processing elements (b) the instar form of processing elements

\section{Competitive Networks}

Fig. 2.11 illustrates the interconnection that implements competition among the instars. The unit activations are determined by differential equations and simplest form is defined by

$$
\dot{x}_{i}=-A x_{i}+\left(B-x_{i}\right)\left[f\left(x_{i}\right)+n e t_{i}\right]-x_{i}\left[\sum_{k \neq i} f\left(x_{k}\right)+\sum_{k \neq i} n e t_{k}\right]
$$

\section{The Outstar}

Fig. 2.12 shows an outstar. It is composed of all of the units in CPN outer layer and a single hidden-layer unit. During the training process, the output values of the outstar can be calculated from

$$
\dot{y}_{i}=-\dot{y}_{i}+b y_{i}+\text { cnet }_{i} \text {. }
$$




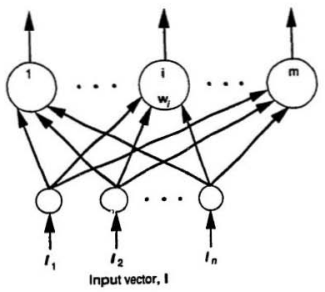

Figure 2.11: A layer of instars arranged in c CPN

\subsubsection{Training the CPN}

The training procedure of CPN can be summarized in the following points:

1. An input vector is selected from all the input vectors to be used for the training.

2. Input vector is normalized and is applied to the GPN competitive layer.

3. The winner should be determined .

4. For the winning unit, only, $\alpha(x-w)$ should be calculated and unit's weight should be updated according to the following equation:

$$
\mathbf{w}(t+1)=\mathbf{w}(t)+\alpha(\mathbf{x}-\mathbf{w})
$$



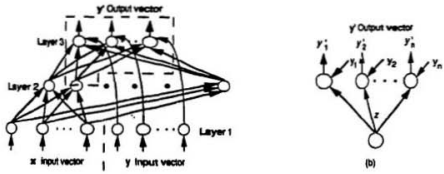

(b)

(a)

Figure 2.12: Outstar and its relationship to the CPN architecture. (a). Outstar structures in CPN network (b). A single outstar unit is shown

5. Steps 1 through 4 should be repeated until all input vectors have been processed once.

6. Step 5 should be repeated until all input vectors have been classified properly.

7. The network should be tested to see the effectiveness.

\subsubsection{Forward Mapping}

It has been assumed that all training has occurred and that the network is now in a production mode. For the input vector $I$ it is necessary to find the corresponding $\mathbf{Y}$ vector. The required processing can be done by the following algorithm: 
1. The input vector should be normalized, $x_{i}=I_{i} /\left(\sqrt{\sum_{n} I_{n}^{2}}\right)$

2. Input vector should be applied to the $x$-vector portion of layer 1 and a zero vector should be applied to the $\mathbf{y}$-vector portion of the same layer.

3. Since the input vector is already normalized, the input layer only distributes it to the units on layer 2.

4. Layer 2 is a winner-take-all competitive layer. The output of each unit can be calculated as follows

$$
z_{i}=\left\{\begin{array}{l}
1, \quad \| \text { net }_{i}>\| \text { net }_{j} \| \text { for all } j \neq i \\
\mathbf{0}, \text { otherwise }
\end{array}\right.
$$

5. The single winner on layer 2 excites an outstar

\subsection{Self-Organizing Maps}

In Self-Organizing Maps(SOM), the CPN network is modified such that, during the learning process, the positive feedback will extend from the central(the winning) unit to the other units in some finite neighborhood around the central unit [18]. In the competitive layer of the CPN, only the winning unit was allowed to learn; in the SOM, all the units in the neighborhood that receive positive feedback from the winning unit participate in the learning process. 


\subsubsection{Unit Activations}

The following equation defines the activation of the processing elements

$$
\dot{y}_{i}=-r_{i}\left(y_{i}\right)+n e t_{i}+\sum_{j} z_{i j} y j
$$

The function $r_{i}\left(y_{i}\right)$ is a general form of a loss term. If $z_{i j}$ takes the form of the Mexicanhat function, then the network will exhibit a bubble of activity around the unit with the largest value of net input.

\subsubsection{The SOM Learning Algorithm}

The learning process can be defined by the following equation

$$
\dot{w}_{i}=\alpha(t)\left(x-w_{i}\right) U\left(y_{i}\right)
$$

where the $w_{i}$ is the weight vector of the ith unit and $\mathbf{x}$ is the input vector. For an input vector $\mathrm{x}$, the winning unit can be determined by

$$
\left\|\mathbf{x}-\mathbf{w}_{c}\right\|=\min _{i}\left\|\mathbf{x}-\mathbf{w}_{\mathbf{i}}\right\|
$$

where index $c$ refers to the winning unit. This can be explained as

$$
\mathbf{w}_{i}(t+1)= \begin{cases}\mathbf{w}_{i}(t)+\alpha(t)\left(x-\mathbf{w}_{\mathrm{i}}(\mathbf{t})\right. & i \in N_{\mathrm{c}} \\ 0 & \text { otherwise }\end{cases}
$$

\subsection{Spatiotemporal Pattern Classification}

Neural networks as described previously are suitable for the recognition of spatial information patterns. Spatiotemporal pattern classifier can classify 


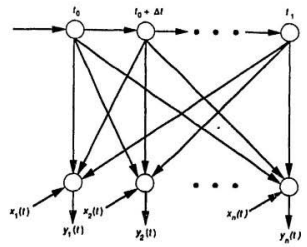

Figure 2.13: Grossberg's formal avalanche structure

time-correlated sequence of spatial patterns [18].

\subsubsection{The Formal Avalanche}

The foundation for the deveiupment of the network architectures discussed in this section is the formal avalanche structure by Grossberg as shown in Fig. 2.13.

\subsubsection{Architectures of Spatiotemporal Networks(STNS)}

Fig. 2.14 shows an arrangement that generates the spatiotemporal patterns (STPs) from spoken word. At each instant of time, the output of the spectrum analyzer consists of a vector whose components are the powers in the 


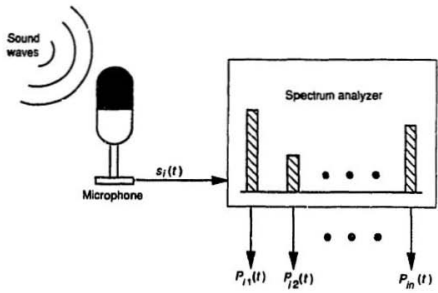

Figure 2.14: Power Spectra generated from speech

various channels.

\subsection{The Neocognitron}

This is a special type of neural network tailored for the recognition of hand written characters [18]. The main pathways for neuron leading from the retina back to area of the brain as the visual, or striate, cortex.

\subsubsection{Neocognitron Architecture}

The processing elements(PEs) of the neocognitron are organized into modules that shall refer to as levels.Each level consists of two layers: a layer of simple cells, or S-cells, followed by a layer of complex cells, or c-cells. 


\subsubsection{Neocognitron Data Processing}

\section{S-cell Processing}

Here it has been considered that the index $k_{l}$ refers to the $k$ th plane on level 1. Each cell on a plane can be labeled with a two-dimensional vector, with $\mathbf{n}$ indicating its position on the plane and $\mathbf{v}$ refer to the relative position of a cell in the previous layer lying in the receptive field of the unit $n$. The equation for the S-cell can be written as :

$$
U_{s l}\left(k_{l}, \mathbf{n}\right)=r_{l} \phi\left[\frac{1+\sum_{k_{l-1}=1}^{k_{l-1}} \sum_{v e A_{l}} a_{l}\left(k_{l-1}, v, k_{l}\right) \cdot U_{C_{l-1}}\left(k_{l-1}, \mathbf{n}+v\right)}{1+\frac{r}{1+r_{l}} b_{l}\left(k_{l}\right) \cdot V_{C l} n}\right]
$$

where the function $\phi$ is a linear function given by

$$
\phi(x)= \begin{cases}x & x \geq 0 \\ 0 & x<0\end{cases}
$$

\section{C-cell Processing}

Usually, units on a given C-plane receive input connections from one, or at most a small number of S-planes on the preceding layer. The output of a C-cell is given by

$$
U_{C l}\left(k_{l}, n\right)=\psi\left[\frac{1+\sum_{k_{l}=1}^{k_{l}} j l\left(k_{l}, k_{l}\right) \sum_{v e D_{l}} d_{l}(v) \cdot U_{l l}\left(k_{l}, \mathbf{n}+\mathbf{v}\right)}{1+V_{u l}(n)}-1\right]
$$


The function $\psi$ is defined by

$$
\psi(x)= \begin{cases}\frac{x}{\beta+x} & x \geq 0 \\ 0 & x<0\end{cases}
$$

Where $\beta$ is constant.

\subsection{Adaptive Resonance Theory(ART)}

Adaptive resonance theory(ART) is an extension of the competitive-learning schemes(CPN) [19]. A key to solving the stability-plasticity dilemma is to add a feedback mechanism between the competitive layer and the input layer of a network. This feedback mechanism facilitates the learning of new information without destroying old information, automatic switching between stable and plastic modes, and stabilization of the encoding of the classes done by the nodes. The results from this approach are two neural network architectures that are particularly suited for the pattern classification problem in realistic environment. These network architectures are ART1 and ART2. ART1 and ART2 differ in the nature of their input patterns. ART1 networks require that the input vectors be binary. ART2 networks are suitable for processing analog or gray-scale patterns [20].

\subsubsection{ART Network Description}

Fig. 2.15 shows the basic features of the ART architecture. There are two types of memory, 


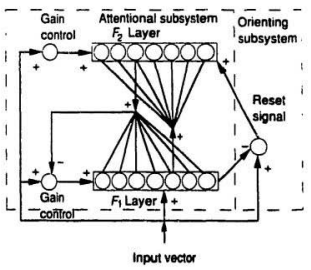

Figure 2.15: Model of ART system

1. short term memory(STM) that develops over the nodes in the two layers.

2. long term memory(LTM), top-down and bottom-up weight between $F_{1}$ and $F_{2}$ layers.

Pattern Matching in ART

The pattern matching cycle in ART can be defined by Fig. 2.16 [20]. 


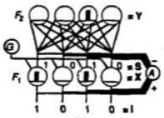

(a)

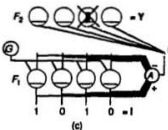

(e)

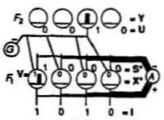

(b)

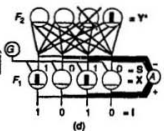

(d)

Figure 2.16: A pattern matching cycle in an ART. (a) pattern-matching attempt (b) reset in (c) final recognition (d) end of matching cycle

\subsubsection{ART1}

The input vector to ART1 is binary and it shares the common architecture of the ART. It's processing can summarized in the following points:

1. Input vector $I$ is applied to $F_{1} . F_{1}$ activities are calculated as follows

$$
x_{1 i}=\frac{I_{i}}{1+A_{1}\left(I_{i}+B_{1}\right)+C_{1}}
$$

2. The output vector for $F_{1}$ is calculated as

$$
s_{i}=h\left(x_{1 i}\right)= \begin{cases}1 & x_{1 i}>0 \\ 0 & x_{1 i} \leq 0\end{cases}
$$

3. $\mathbf{S}$ is propagated forward to $F_{2}$ and the activities are calculated as

$$
T_{j}=\sum_{i=1}^{M} s_{i} z_{j i}
$$


4. Only the winning $F_{2}$ node has a nonzero output:

$$
u_{i}= \begin{cases}1 & T_{j}=\max _{k} T_{k} \forall \\ 0 & \text { otherwise }\end{cases}
$$

5. Output from $F_{2}$ is propagated back to $F_{1}$. Net inputs from $F_{2}$ on the units of $F_{1}$ are calculated as

$$
V_{i}=\sum_{j=1}^{N} u_{j} z_{i j}
$$

6. New activities are calculated as

$$
x_{1 i}=\frac{I_{i}+D_{1} V_{i}-B_{1}}{1+A_{1}\left(I_{i}+D_{1} V_{i}\right)+C_{1}}
$$

7. As in step 2 out on values $s_{i}$ is calculated.

8. The degree of match between the input pattern and the top-down template is given by the following equation

$$
\frac{|S|}{|I|}=\frac{\sum_{i=1}^{M} s_{i}}{\sum_{i=1}^{M} I_{i}}
$$

9. If $|S| /|I|<\rho$, then $v_{J}$ is marked as inactive, zero the outputs of $F_{2}$. and it is necessary to return to stepl. If not then we have to continue.

10. Bottom-up weight has to be updated on $v_{J}$ only

$$
z_{J i}= \begin{cases}\frac{L}{L-1+|\sigma|} & \text { if } v_{i} \text { is active } \\ 0 & \text { if } v_{i} \text { is inactive }\end{cases}
$$

11. Top-down weight is updated coming from $v_{J}$ only to all $F 1$ units

$$
Z_{i J}= \begin{cases}1 & \text { if } v_{i} \text { is active } \\ 0 & \text { if } v_{i} \text { is inactive }\end{cases}
$$

12. Input pattern should be removed. All inactive $F \mathbf{2}$ units should restored 


\subsubsection{ART2}

This neural network is similar to ART1. It only differs in the sense that its input pattern is analog signal.

\subsection{Comparative analysis and selection of suitable Network}

In case of incipient fault detection of induction motor based on spectral recognition, an ANN should have the following properties:

1. Low training time.

2. Ability to learn new knowledge while retaing the old one without any retraining of past pattern

3. Traning process should have certainity to reach global minima.

4. It should accept analog input pattern.

5. Input pattern should be in spatial domain

6. It should have ability to report if it can not classify a particular pattern.

7. It should be a general purpose ANN, so that necessary modifications can be done to make it suitable to the present problem domain. 
To select a proper ANN for this purpose salient features of different ANNs have been summarized in the following Table. From the previous discussion it can be concluded that ART2 satisfies the necessary characteristics to be accepted as suitable neural network in incipent fault detection of an induction motor. Detailed software implementation and performance of ART2 are explained in the next chapter.

\section{Table-2.1}

Comparative Performances of Different ANN Techniques.

\begin{tabular}{|c|c|c|c|c|c|c|c|}
\hline $\begin{array}{c}\text { Type of } \\
\text { ANS }\end{array}$ & $\begin{array}{c}\text { Training } \\
\text { umase }\end{array}$ & 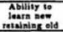 & $\begin{array}{l}\text { Cembinify so } \\
\text { reach plobeal } \\
\text { miains. }\end{array}$ & $\begin{array}{l}\text { Type et } \\
\text { inpei } \\
\text { patierts }\end{array}$ & $\begin{array}{l}\text { Demain } \\
\text { iapai } \\
\text { polleter }\end{array}$ & $\begin{array}{l}\text { Abrisy io } \\
\text { meport if } \\
\text { mikchmaich }\end{array}$ & Perpent \\
\hline BPN & Pint & No & So & Anaver & Spatia & No & Oeneter \\
\hline ixM & Los & No & Bio & Dirite & Spaila & s. & Oenenta \\
\hline H4 & $\operatorname{lom}$ & W. & Bo & Dirisa & Spotia & wo & Oentere \\
\hline EM & Hint & No & 80 & Diride & Spatia & No & Oanenal \\
\hline CPN & $L_{00}$ & No & B० & Dirial & Spotia & Ho & Conental \\
\hline SOM & Moderait & No & Mo & Amaing & Bpaln & 76 & Conene \\
\hline 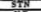 & Modertie & No & Y. & Anelof & inmpers & प्र० & Conered \\
\hline NC & Moderise & No & 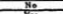 & Analor & spatia & 80 & Spechenal \\
\hline ARt7 & Lar & Yoi & Yes & Dirial & Spatial & $Y_{00}$ & Ganentel \\
\hline AETY & ton & Yet & Yat & Analor & Spand & Yen & Comental \\
\hline
\end{tabular}




\section{Chapter 3}

\section{ART2 Neural Network}

\subsection{Introduction}

In response to the questions of dynamic updating and training time of conventional neural network, adaptive resonance theory(ART) has been proposed by Grossberg, Carpenter and others [19] . ART2 is a special version of ART having the property of analog input. In the implementation phase it has been modified to have an additional property of reporting if it cannot find a match for an input pattern.

\subsection{ART2 Architecture}

The structure of ART2 can be represented by Fig. 3.1. It consists of two subsystem known as attentional subsystem(AS) and orienting subsystem(OS). The AS consists of two layers of processing elements (PEs), F1 and $F 2$ and 


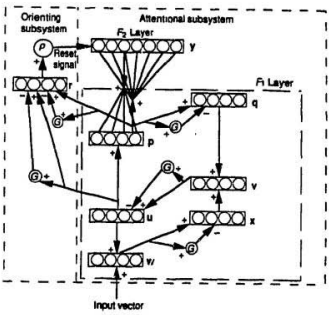

Figure 3.1: The overall structure of ART2

a gain-control system $\mathrm{G}$.

\subsubsection{The Attentional Subsystem}

The activities of processing elements on the layers $F 1$ and $F 2$ can be defined by the following dynamic equation as

$$
\varepsilon \dot{x}_{k}=-x_{k}+\left(1-A x_{k}\right) J_{k}^{+}-\left(B+C x_{k}\right) J_{k}^{-}
$$

where $J_{k}^{+}$and $J_{k}^{-}$are the excitory and inhibitory inputs to the $k$ th unit, respectively. The precise definition of $A, B$ and $C$ depends upon the layer and for this case $B$ and $C$ have been considered to be zero. Here it has been considered that $x_{1 i}$ and $x_{2 j}$ refer to the activities on $F 1$ and $F 2$ layers, respectively, Here $v_{i}$ represents the nodes on $F 1$ and $v j$ those on $F 2$. The 


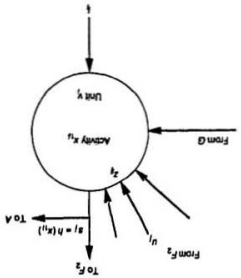

Figure 3.2: Structure of processing element on $F_{1}$ layer

constant $\varepsilon$ determines how fast $x_{k}$ reaches to equilibrium.

\section{Processing on F1}

A single processing element on $F 1$ with its v vious inputs and weight vectors can be represented by Fig. 3.2 [19]. The units calculate a net-input value coming from $F 2$ in the usual way:

$$
V_{i}=\sum_{i} u_{j} z_{i j}
$$

The values of indivitual quantities in the defining equations of $F 1$ and $F 2$ vary according to the sublayer being considered. For the sake of convenience, the appropriate values of the parameters for layer $F 1$ have been 
summarized in the Table 3.1. Based on the table, the activities on each of the six sublayers on $F 1$ can be summarized by the following equations:

Table 3.1

Values of parameters on F-1 layer

\begin{tabular}{|c|c|c|c|c|}
\hline & \multicolumn{4}{|c|}{ Quantity } \\
\hline Layer & $A$ & D & $s_{i}^{+}$ & $J_{i}^{-}$ \\
\hline$w$ & 1 & ? & $I_{1}+a u_{1}$ & 0 \\
\hline$x$ & e & 1 & $w$ & $\|w\|$ \\
\hline $\mathbf{u}$ & e & 1 & v, & $\|\mathbf{v}\|$ \\
\hline $\mathbf{v}$ & $t$ & $t$ & $f\left(x_{i}\right)+b f\left(q_{i}\right)$ & 0 \\
\hline p & I & 1 & $u_{1}+\sum_{z} g(y,) z_{i j}$ & 0 \\
\hline q & e & 1 & $p_{n}$ & $\|\mathbf{l}\|$ \\
\hline $\mathbf{r}$ & e & 1 & $u_{i}+c p_{i}$ & $\|\mathbf{u}\|+\mathbf{c p} \|$ \\
\hline
\end{tabular}

$$
\begin{gathered}
w_{i}=I_{i}+a u_{i} \\
x_{i}=\frac{w_{i}}{e+\|\mathbf{w}\|} \\
v_{i}=f\left(x_{i}\right)+b f\left(g_{i}\right) \\
u_{i}=\frac{v_{i}}{e+\|\mathbf{v}\|} \\
p_{i}=u_{i}+\sum_{j} g\left(y_{i}\right) z_{i j}
\end{gathered}
$$




$$
q_{i}=\frac{p_{i}}{e+\|\mathbf{p}\|}
$$

The form of the function $f(x)$ determines the nature of the contrast enhanchement that takes place on $\boldsymbol{F}_{1}$. A sigmoid might be the logical choice for this function, but Carpenter's [20] choice is

$$
f(x)= \begin{cases}0 & 0 \leq x \leq \theta \\ x & x>\theta\end{cases}
$$

where $\theta$ is a positive constant less than one.

\section{Processing on F2}

Fig. 3.3 shows a typical $\mathrm{PE}$ on $F_{2}$ layer. Bottom-up weights are calculated according to the following equation

$$
T_{j}=\sum_{i} p_{i} z_{j i}
$$

The output on $F 2$ is given by the function

$$
g\left(y_{i}\right)= \begin{cases}d & T_{j}=\max _{k} T_{k} \\ 0 & \text { otherwise }\end{cases}
$$

\subsubsection{The Orienting Subsystem}

From the parameter table and the defining equation of ART2, the actiuvites on the layer $\mathbf{r}$ on the orienting subsystem can be defined by

$$
r_{i}=\frac{u_{i}+c p_{i}}{\|\mathbf{u}\|+\|c \mathbf{p}\|}
$$




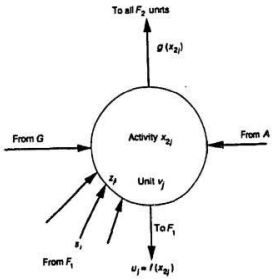

Figure 3.3: Structure of processing element in $F_{2}$ layer

Here it has been assumed that $e=0$ and the condition for reset is

$$
\frac{\rho}{\|\mathbf{r}\|}>1
$$

It should be noted that two sublayers $\mathbf{p}$ and $\mathbf{u}$ participate in the matching process. As top-down weight changes on the $\mathbf{p}$ layer during learning, the activity of the units on the $\mathbf{p}$ layer also changes. The $\mathbf{u}$ layer remains stable during this process, so including it in the matching process prevents reset from occurring while learning of a new pattern is taking place.

\subsubsection{Gain Control in ART2}

The three gain control units on $F 1$ nonspecifically inhibit the $\mathrm{x}, \mathrm{u}$, and $\mathrm{q}$ sublayers. The inhibitory signal is equal to the magnitude of the input vector 
to those layers. The effect is that the activities of these three layers are normalized to unity by the gain control signals.

\subsubsection{Least-mean-square Equations}

Both bottom-up and top-down Least-mean-square equations have the same form as shown below:

$$
\dot{z}_{j i}=g\left(y_{i}\right)\left(p_{i}-z_{j i}\right)
$$

for the bottom-up weights from $v_{i}$ on $F_{1}$ to $v_{j}$ on $F_{2}$, and

$$
\dot{z}_{j i}=g\left(y_{i}\right)\left(p_{i}-z_{j i}\right)
$$

for top-down weights from $v_{j}$ on $F_{2}$ to $v_{i}$ on $F_{1}$. If $v_{J}$ is winning node, then from the previous equations it can be shown that

$$
\dot{z}_{d i}=d\left(u_{i}+d z_{i J}-z_{J i}\right)
$$

and similiarly

$$
\dot{z}_{i J}=d\left(u_{i}+d z_{i J}-z_{i J}\right)
$$

with all other $\dot{z}_{i j}=\dot{z}_{j i}=0$ for $j \neq J$. For the fast-learning case for the equilibrium values of the weights:

$$
z_{J i}=z_{i J}=\frac{u_{i}}{1-d}
$$


where it has assumed that $0<d<1$

\subsubsection{Bottom-Up Least-mean-square Initialization}

The bottom-up weight vectors can be initialized by the following relation:

$$
\|z\|<\frac{1}{1-d}
$$

It is also possible to accomplish the initialization process by setting the weights to small random numbers. Alternatively, the initialization process can be performed also by the relation

$$
z_{j i}(0) \leq \frac{1}{(1-d) \sqrt{ } M}
$$

\subsubsection{ART2 Processing Summary}

In this situation only the asymtotic solutions to the dynamic equations, and. the fast-learning mode have been considered and it has also been considered that $M$ be the number of units in each $F_{1}$ sublayer, and $N$ be the number of units on $F_{\mathbf{2}}$. Parameters are chosen according to the relations $a, b>0 ; 0 \leq d \leq 1 ; \frac{c d}{1-d} \leq 1 ; 0 \leq \theta \leq 1 ; 0 \leq \rho \leq 1 ; e<<1$.Top-down weights are initialized to zero $z_{i j}=0$ and bottom-up weights are initialized according to

$$
z_{i j}(0) \leq \frac{1}{(1-d) \sqrt{ } M}
$$

The processing can be now summarized in the following points [18]: 
1. It is necessary to initialize all layer and sublayer outputs to zero vectors, and to establish a cycle counter to a value of one.

2. An input pattern $\mathbf{I}$ should be applied to the w layer on $F_{1}$. The output of this layer is

$$
w_{i}=I_{i}+a u_{i}
$$

3. Forward propagation to the $\mathbf{x}$ sublayer should be done:

$$
x_{i}=\frac{w_{i}}{c+\|w\|}
$$

4. Propagation forward to the $\mathrm{v}$ sublayer is done

$$
v_{i}=f\left(x_{i}\right)+b f\left(q_{i}\right)
$$

5. The result should be propagated to the $\mathbf{u}$ sublayer,

$$
u_{i}=\frac{v_{i}}{c+\|v\|}
$$

6. Forward propagation to the $\mathbf{p}$ is done by

$$
p_{i}=u_{i}+d z_{i J}
$$

where the $J$ th node on the $F_{2}$ is the winner of the competition on that layer. If $F_{2}$ is inactive, $p_{i}=u_{i}$.

7. It is necessary to propagate to the q sublayer

$$
q_{i}=\frac{p_{i}}{e+\|\mathbf{p}\|}
$$

8. Steps 2 through 7 should be repeated to stabilize the values on $F_{1}$ 
9. The output of $\mathbf{r}$ layer is calculated

$$
r_{i}=\frac{u_{i}+c p_{i}}{e+\|\mathbf{u}\|+\|c \mathbf{p}\|}
$$

10. Whether a reset condition is indicated should be determined. If $\rho /(e+$ $\|\mathbf{r}\|)>1$, then a reset signal to $F_{2}$ should be sent. Any active node on $F_{2}$ should be marked ineligible for the competetion and the cycle counter should be set to one and should be returned to step 2 . If there is no reset, and the cycle counter is one, cycle counter should be incremented and continue with step 11. If there is no reset, and the cycle counter is greater than one, then it necessary to skip to step 14 , as resonance has been established.

11. The output of the $\mathbf{p}$ sublayer should be propagated to the $F_{2}$ layer and the net inputs to $F_{2}$

$$
T_{j}=\sum_{i=1}^{M} p_{i} z_{j i}
$$

12. Only the winning F2 node has nonzero output.

$$
g\left(T_{j}\right)= \begin{cases}d & T_{j}=\max _{k} T_{k} \\ 0 & \text { otherwise }\end{cases}
$$

13. Step 6 through 10 should be repeated.

14. Bottom-up weights on $F_{2}$ unit should be modified

$$
z_{J i}=\frac{u_{i}}{1-d}
$$

15. Top-down weights from the winning $F_{2}$ unit should be modified

$$
z_{i j}=\frac{u_{i}}{1-d}
$$


16. Input vector should be removed and all inactive $F_{2}$ units should be restored and now it is the time to return to step 1 with new input pattern.

\subsection{ART2 Simulator}

ART2 has been implemeted using Object Oriented software development methodology. It has been considerer as an independent class structure so that an instance of this class can be easily used to an application software like on-line condition monitoring system of induction motor.

\subsubsection{Model of ART2 as an Object}

The model of ART2 as an object can be shown in Fig. 3.4. Salient features of this object are pattern encoding and decoding through training and recalling, dynamic addition and deletion of neurons in the network. Fig. 3.5 shows the flow chart of training algorithm of ART2.The detailed program listing is given in Appendix-A.

\subsubsection{Modified Structure of Training and Recalling Pattern in the Network}

To add the property to give a unmatched signal if it cannot find a pattern in the network and also to to make the training process faster, the pattern encoding and decoding technique has been slightly modified as shown in Fig. 3.6. 


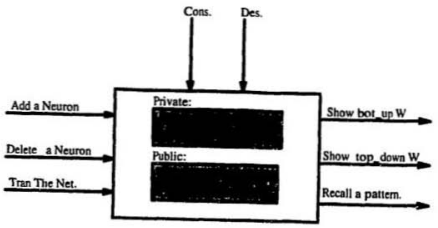

Figure 3.4: Model of ART2 as an object

\subsubsection{Dynamic Updating}

In the object model of the network, it should be noted that there are provisions for addition and deletion of nodes. So, the structure of the ANN is not determined by the initial parameters. Due to this advantage, ART2 type neural network is suitable for a dynamic scenario like incipient fault detection, which does not require retraining of the already trained patterns. Moreover, using this dynamic property it is possible to make optimum use of the computing resources.

\subsection{Experimental Varification of Performance}

For the experimental purpose, the number of neurons in layer $F_{2}$ and $F_{1}$ have been considereset to 6 and 9, respectively. The following parameters have been initialized with specified values:

$a=10 ; b=0.10 ; c=0.08 ; \theta=0.2 ; e=0.0 ; \rho=0.995$. Initial bottom-up weight $=2.08$, initial top-down weight $=0.0$. 


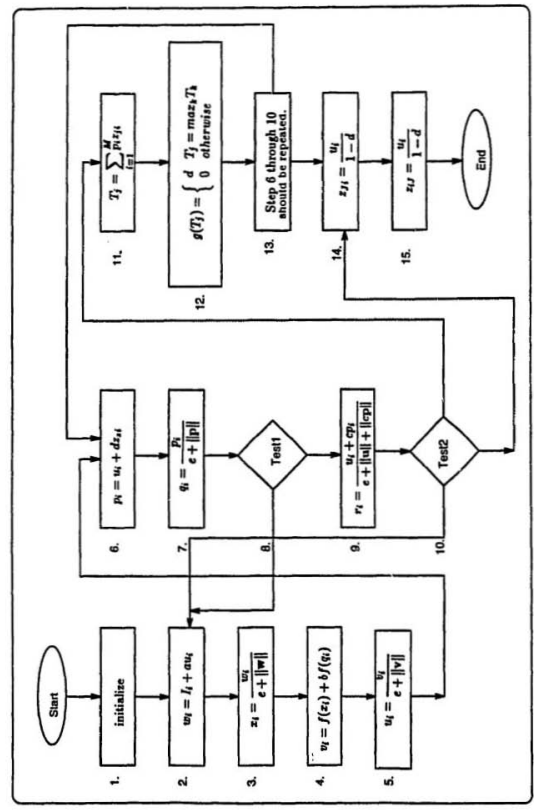

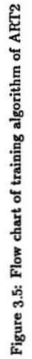




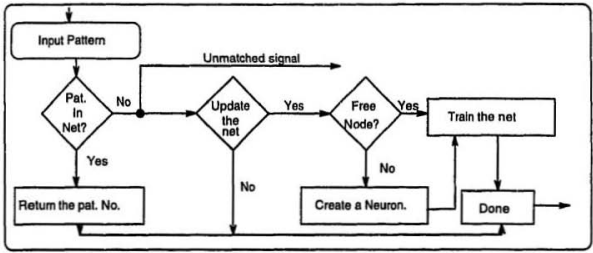

Figure 3.6: Modified Training and Recall Algorithm of ART2

\subsubsection{Training of the Neural Network with test Patt- tern}

Pattern of the exemplars and corresponding training results are shown in Table 3.2. Table 3.3 and Table 3.4 represent the bottom-up and top-adrn weight matrices, respectively. In the training session it should be noted that the network has stored both the patterns 2 and 3 in the same class, though the vectors are seperated by euclidian distance $\sqrt{(65-60)^{2}+(15-13)^{2}}=5.38$. This type of behaviour is suitable to classify like patterns in same class to realize fuzzy nature of the problem. But when two vectors are far apart as in case of patterns 3 and 4, the network classifies them in seperate classes. 
Table 3.2

Set of Training examples Used to Train ART2.

\begin{tabular}{|c|c|c|}
\hline No. & Pattern: & Training Result. \\
\hline 1. & $\begin{array}{lllllllllll}60.0 & 0.0 & 20.0 & 11.0 & 20.0 & 0.0 & 10.0 & 0.0 & 18.0\end{array}$ & stored in class 0 \\
\hline 2. & $\begin{array}{lllllllll}60.0 & 16.0 & 31.0 & 0.0 & 17.0 & 0.0 & 0.0 & 0.0 & 13.0\end{array}$ & stored in class 1 \\
\hline 3. & $\begin{array}{lllllllll}65.0 & 16.0 & 31.0 & 0.0 & 17.0 & 0.0 & 0.0 & 0.0 & 15.0\end{array}$ & stored in class 1 \\
\hline 4. & $\begin{array}{lllllllll}50.0 & 11.0 & 31.0 & 0.0 & 0.0 & 0.0 & 23.0 & 0.0 & 12.0\end{array}$ & stored in class 2 \\
\hline 5. & 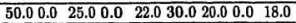 & stored in class 3 \\
\hline 6. & $\begin{array}{lllllllll}67.0 & 25.0 & 44.0 & 15.0 & 0.0 & 10.0 & 11.0 & 0.0 & 17.0\end{array}$ & stored in class 4 \\
\hline 7. & $68.026 .0 \quad 48.021 .020 .0 \quad 0.0 \quad 0.0 \quad 10.00 .0$ & stored in class 5 \\
\hline 8. & $17.027 .042 .016 .027 .020 .0 \quad 40.0 \quad 0.0 \quad 13.0$ & No more neuron \\
\hline
\end{tabular}

Table 3.3

Bottom-up weight matrix after training.

\begin{tabular}{|l|l|l|l|l|l|}
\hline \hline 10.19 & 10.50 & 9.89 & 8.64 & 9.97 & 9.25 \\
\hline 0.0 & 2.80 & 0.0 & 0.0 & 3.72 & 3.54 \\
\hline 3.64 & 5.42 & 6.13 & 4.32 & 6.55 & 6.53 \\
\hline 0.0 & $\mathbf{0 . 0}$ & 0.0 & 0.0 & 0.0 & 2.86 \\
\hline 3.64 & 2.97 & 0.0 & 3.80 & 0.0 & 2.72 \\
\hline 0.0 & $\mathbf{0 . 0}$ & 0.0 & 5.18 & 0.0 & 0.0 \\
\hline 0.0 & $\mathbf{0 . 0}$ & 4.55 & 3.46 & 0.0 & 0.0 \\
\hline 0.0 & $\mathbf{0 . 0}$ & 0.0 & 0.0 & 0.0 & 0.0 \\
\hline 3.27 & $\mathbf{0 . 0}$ & 0.0 & 3.11 & 0.0 & 0.0 \\
\hline
\end{tabular}

Table 3.4

Top-down weight matrix after training.

\begin{tabular}{|l|l|l|l|l|l|l|l|l|}
\hline \hline 10.91 & 0.0 & 3.64 & 0.0 & 3.64 & 0.0 & 0.0 & 0.0 & 3.27 \\
\hline 10.50 & 2.80 & 5.42 & 0.0 & 2.97 & 0.0 & 0.0 & 0.0 & 0.0 \\
\hline 9.89 & 0.0 & 6.13 & 0.0 & 0.0 & 0.0 & 4.55 & 0.0 & 0.0 \\
\hline 8.64 & 0.0 & 4.32 & 0.0 & 3.80 & 5.18 & 3.46 & 0.0 & 3.11 \\
\hline 9.97 & 3.72 & 6.55 & 0.0 & 0.0 & 0.0 & 0.0 & 0.0 & 0.0 \\
\hline 9.25 & 3.54 & 6.53 & 2.86 & 2.72 & 0.0 & 0.0 & 0.0 & 0.0 \\
\hline \hline
\end{tabular}




\subsubsection{Dynamic Neuron Addition}

As number of neurons in the output layer $F_{2}$ is 6 , so it can classify only six classes of patterns. Now in case of a dynamic system the network should have the ability to update it to handle this new situation. Using Add-Neuron behaviour of the network this can be accomplished as shown in Table 3.5 after addition of a new neuron. No retraining of previous patterns is necessary. Now, after the addition of last pattern new bottom-up and top-down weight matrices are given in Table 3.6 and Table 3.7, respectively.

Table 3.5

New training vector to train after neuron addition.

\begin{tabular}{|c|c|c|}
\hline No. & Pattern: & Training Result. \\
\hline 8. & $17.027 .042 .0 \quad 16.027 .020 .040 .00 .0 \quad 13.0$ & stored in class 6 \\
\hline
\end{tabular}

Table 3.6

Bottom-up weight matrix after training.

\begin{tabular}{|l|l|l|l|l|l|l|}
\hline \hline 10.19 & 10.50 & $\mathbf{9 . 8 9}$ & 8.64 & 9.97 & 9.25 & 2.80 \\
\hline 0.0 & 2.80 & $\mathbf{0 . 0}$ & 0.0 & 3.72 & 3.54 & 4.44 \\
\hline 3.64 & 5.42 & 6.13 & 4.32 & 6.55 & 6.53 & 6.91 \\
\hline 0.0 & 0.0 & 0.0 & 0.0 & 0.0 & 2.86 & 2.63 \\
\hline 3.64 & 2.97 & $\mathbf{0 . 0}$ & 3.80 & 0.0 & 2.72 & 4.44 \\
\hline 0.0 & 0.0 & 0.0 & 5.18 & 0.0 & 0.0 & 3.29 \\
\hline 0.0 & 0.0 & 4.55 & 3.46 & 0.0 & 0.0 & 6.58 \\
\hline 0.0 & 0.0 & 0.0 & 0.0 & 0.0 & 0.0 & 0.0 \\
\hline 3.27 & 0.0 & 0.0 & 3.11 & 0.0 & 0.0 & 0.0 \\
\hline
\end{tabular}




\section{Table 3.7}

Top-down weight matrix after training.

\begin{tabular}{|l|l|l|l|l|l|l|l|l|}
\hline 10.91 & 0.0 & 3.64 & 0.0 & $\mathbf{3 . 6 4}$ & 0.0 & 0.0 & $\mathbf{0 . 0}$ & 3.27 \\
\hline 10.50 & 2.80 & 5.42 & $\mathbf{0 . 0}$ & $\mathbf{2 . 9 7}$ & 0.0 & 0.0 & $\mathbf{0 . 0}$ & 0.0 \\
\hline 9.89 & 0.0 & 6.13 & $\mathbf{0 . 0}$ & $\mathbf{0 . 0}$ & 0.0 & 4.55 & $\mathbf{0 . 0}$ & 0.0 \\
\hline 8.64 & 0.0 & 4.32 & $\mathbf{0 . 0}$ & $\mathbf{3 . 8 0}$ & 5.18 & 3.46 & $\mathbf{0 . 0}$ & 3.11 \\
\hline 9.97 & 3.72 & 6.55 & $\mathbf{0 . 0}$ & 0.0 & 0.0 & 0.0 & $\mathbf{0 . 0}$ & 0.0 \\
\hline 9.25 & 3.54 & 6.53 & $\mathbf{2 . 8 6}$ & 2.72 & 0.0 & 0.0 & $\mathbf{0 . 0}$ & 0.0 \\
\hline 2.80 & 4.44 & 6.91 & $\mathbf{2 . 6 3}$ & 4.44 & 3.29 & 6.58 & $\mathbf{0 . 0}$ & 0.0 \\
\hline \hline
\end{tabular}

\subsubsection{Pattern Recall from the Network}

The performances of the network in pattern matching are given in Table 3.8. From Table 3.8, pertinent information can be highlighted. In case of pattern 1 , the euclidian distance of the recall pattern from the closest trained pattern is $\sqrt{(5.0-0.0)^{2}+(15-10)^{2}}=7.07$, but the network has classified it as member of class 0 . But when the distance is very high as in case of patterne 5 and 7 , the network has reported that it is unable to classify, instead of giving some false classification result. This is one of the most important behaviours from the neural network for the diagnostic purpose of incipient faults of an induction motor. 
Table 3.8

Pattern matching result of the trained network.

\begin{tabular}{|c|c|c|}
\hline No. & Pattern: & Training Result. \\
\hline 1. & 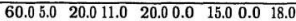 & classified as class 0 \\
\hline 2. & $\begin{array}{lllll}17.0 & 10.0 & 0.0 & 0.0 & 13.0\end{array}$ & classified as class 1 \\
\hline 3. & $\begin{array}{llllll}17.0 & 0.0 & 0.0 & 0.0 & 15.0\end{array}$ & classified as class 1 \\
\hline 4. & 50.011 .031 .00 .0 & classified as class 2 \\
\hline 5. & 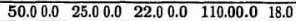 & unable to classify \\
\hline 6. & $\begin{array}{lllllllll}67.025 .0 & 44.0 & 15.0 & 0.0 & 10.0 & 11.0 & 0.0 & 17.0\end{array}$ & classified as class 4 \\
\hline 7. & $\begin{array}{lllllllll}68.0 & 26.0 & 8.0 & 21.0 & 20.0 & 0.0 & 0.0 & 10.0 & 0.0\end{array}$ & unable to classify \\
\hline 8. & 17.027 .042 .016 .027 .020 .0 & classified as class 6 \\
\hline
\end{tabular}




\section{Chapter 4}

\section{Fault Related Information Collection}

\subsection{Introduction}

From study it has been noticed that the waveform of the stator current of an induction motor carries the signature of internal status of the machine [15]. The frequency spectra of the stator current can be considered as the information carrier of the incipient faults [13].

\subsection{Model of Spectra Collection}

The input current to an induction machine is an analog signal. But due to the rapid development of digital signal processing(DSP) techniques, it is comparatively easy now to use these tools to get the frequency spectra of the stator curnent. The model of spectra collection can be explained by the Fig. 4.1 


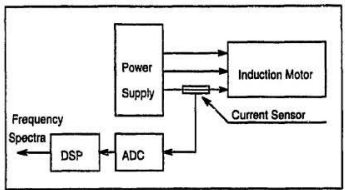

Figure 4.1: Model of Fault Related Spectra Collection

\subsection{Discrete Time Signals and Systems}

A signal can be defined as a function that conveys the information, generally about the state or behaviour of a physical system [21].The independent variable in the mathematical representation of a signal may be either continuous or discrete. Continuous-time signals are defined along a continuum of time and thus are represented by a continuous independent variable. Continuous time signals are often referred to as analog signals. Discrete-time signals are defined at discrete times and thus the independent variable has discrete values. Digital signals are those for which both time and amplitude are discrete. 


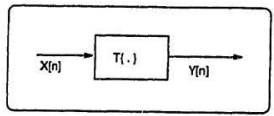

Figure 4.2: Representation of discrete-time system

\subsubsection{Discrete-Time Signals: Sequences}

Discrete-time signals are repronted mathematically as sequences of numbers. A sequence of numbers $x$, in which the $n$th number in the sequence is denoted by $x[n]$, is formally written as

$$
x=x[n],-\infty<n<\infty
$$

where $n$ is an integer.

\subsubsection{Discrete-Time Systems}

A discrete-time system is defined mathematically as a transformation or operator that maps an input sequence with values $x[n]$ into an output sequence with values $y[n][21]$. This can be denoted as

$$
y[n]=T x[r]
$$

and is indicated pictorially in Fig. 4.2. 


\subsubsection{Sampling of Continuous-Time Signals}

Typical method of obtaining a discrete-time signal representation of a continuoustime signal is through periodic sampling, wherein a sequence $x[n]$ is obtained from a continuous-time signal $x_{\mathrm{c}}(t)$ according to the relation

$$
x[n]=x_{c}(n T),-\infty, n, \infty
$$

where $T$ is the sampling period, and its reciprocal, $f_{s}=1 / T$, is the sampling frequency, in samples per second.

Nyquist Sampling Theorem:

Let $x_{c}(t)$ be a band-limited signal with

$$
X_{c}(j \Omega)=0 \text { for }|\Omega|>\Omega_{N}
$$

Then $x_{c}(t)$ is uniquely determined by its samples

$x[n]=x_{c}(n T), n=0, \pm 1, \pm 2, \ldots .$, if

$$
\Omega_{s}=\frac{2 \pi}{T}>2 \Omega_{N}
$$

The frequency $\Omega_{N}$ is commonly referred as the Nyquist frequency, and the frequency $2 \Omega_{N}$ that must be exceeded by the sampling frequency is called the Nyquist rate. 


\subsubsection{The Discrete Fourier Transform(DFT)}

For a finite length sequence $x[n]$ of length $N$ generally the DFT analysis and synthesis equations are written as [21]

Analysis equation: $X[k]=\sum_{n=0}^{N-1} x[n] W_{N}^{k n}, \quad k=0,1, \ldots \ldots, N-1$

$$
\text { Synthesis equation: }:[n]=\frac{1}{N} \sum_{k=0}^{N-1} X[k] W_{N}^{-k n}, n=0,1, \ldots, N-1
$$

Here $W_{N}=e^{-j(2 \pi / N)}$

\subsubsection{Computation of the Discrete Fourier Transform}

The DFT is an important component in many practical applications of discrete-time systems. To make the DFT computation faster a number of efficient algorithms have been developed collectively known as fast Fourier transform(FFT).

\section{Decimination-In-Time FFT Algorithm}

Algorithms in which the decomposition is based on decomposing the sequences $x[n]$ into successively smaller subsequences, are called deciminationin-time algorithms. An (N/2) point DFT can be represented by

$$
X[k]=\sum_{r=0}^{(N / 2)-1} x[2 r] W_{N / 2}^{r k}+W_{N}^{k} \sum_{r=0}^{(N / 2)-1} x[2 r+1] W_{N / 2}^{p k}
$$




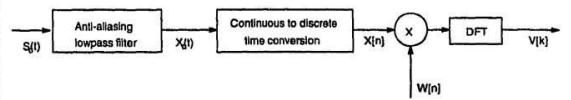

Figure 4.3: Processing steps in the discrete-time Fourier analysis of a continuous- time signal

\section{Decimination-In-Frequency FFT Algorithm}

For a sequence $x[n]$ the output sequence $X[k]$ can be divided into smaller and smaller subsequences in the same manner. FFT algorithms based on this procedure are commonly called decimination-in-frequency algorithm. The $N / 2$ point DFT of the $N / 2$-point sequence can be represented by

$$
X[2 r]=\sum_{n=0}^{(N / 2)-1}(x[n]+x[n+(N / 2)]) W_{N / 2}^{n n}, r=0,1,2, \ldots \ldots,(N / 2)-1
$$

\subsubsection{Fourier Analysis of Signals Using the Discrete Fourier Transform}

The consistency between the finite-length requirement of the DFT and the reality of indefinitely long signals can be accommodated exactly or approximately through the concepts of windowing, block processing, and the timedependent Fourier transform. The processing steps in the discrete Fourier analysis of a continuous time signal can be represented by Fig. 4.3. Fig. 4.4 is the illustration of the Fourier transforms . 

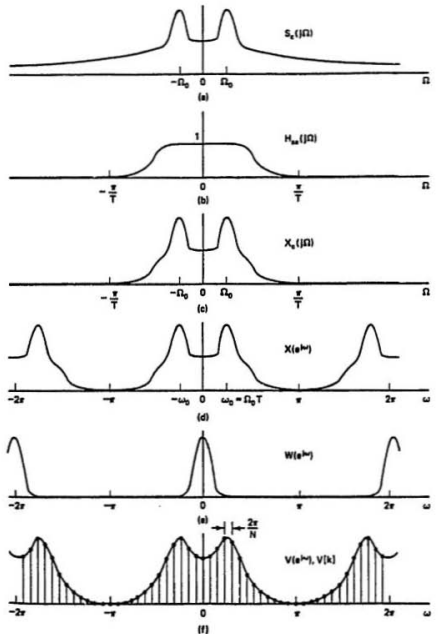

Figure 4.4: Illustration of the Fourier transforms in the system : (a) Fourier transform of continuous-time input signal. (b) Frequency response of anti aliasing filter. (c) Fourier transform output of a ati aliasing filter. (d) Fouvier transform of sampled signal (e) Fourier transfurm of window sequence (f) Fourier transform of windowed signal segment and frequency samples obtained using DFT samples 


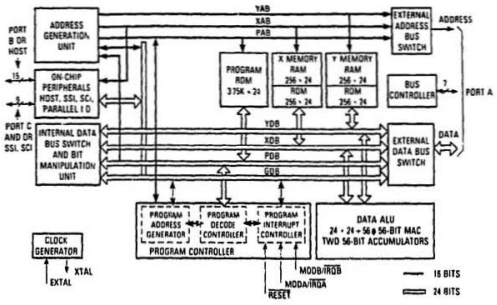

Figure 4.5: DSP56000 Block Diagram

\subsection{Motorola's DSP56000 DSP Family}

The DSP56000 and DSP56001 are the two members of Motorola's Family of HCMOS, low-power, general-purpose DSPs [22]. Block diagram of the DSP56000 is shown in Fig. 4.5. The DSP56001 features 512 words of fullspeed, on-chip, program RAM, two preprogrammed data ROMs, and special on-chip bootstrap hardware to permit convenient loading of user programs into the program RAM. The DSP56001 is an off-the-shelf item since there are no user-programmable on-chip ROMs. The DSP56000 features $3.75 \mathrm{k}$ words of full speed, on-chip, program ROM instead of 512 words of program RAM.

The central part of the processor consists of three execution units operating in parallel:

1. the data arithmetic logic unit(ALU) 
2. the address gencration unit(AGU)

3. the program controller

The DSP5600/DSP56001 has microcontroller unit (MCU)-style on-chip peripherals, program memory, data memory, and a memory expansion port. The microprocessor unit (MPU)-style programming model and instruction set allow straightforward generation of efficient and compact code. The high throughput of the DSP56000/DSP56001 makes it well-suited for communication, high-speed control, numeric processing, computer applications, and audio applications. The main features making this throughput are as follows:

- Speed: At 1025-million instruction per second(MIP), the DSP56000/DSP56001 can execute a 1024-point complex FFT in $3.23 \mathrm{~ms}$.

- Precision: The data paths are 24 bits wide, providing $144 \mathrm{~dB}$ of dynamic range; intermediate results held in the 56-bit accumulators can range over $336 \mathrm{~dB}$.

- Parallelism: Each on-chip execution unit(AGU, program controller,data ALU), memory, and peripheral operates independently and in parallel with the other units through a sophisticated bus system. The data ALU, AGUs and program controller operate in parallel so that an instruction prefetch, a 24-bitx24-bit multiplication, a 56-bit addition, two data moves, and two address pointer updates using one of the three types of arithmetic can be executed in a single instruction cycle. This parallelism allows a four-coefficient infinite impulse response(IIR) filter 
section to be executed in only four cycles, the theoretical minimum for single-multiplier architecture. At the same time, two serial controllers can send and receive full-duplex data, and the host port can send/receive simplex data.

- Integration: In addition to the three independent execution units, the DSP56000/DSP56001 has six on-chip memories, three on-chip MCU. style peripherals (serial communication interface( $\mathrm{SCI}$ ), synchronous serial interface(SSI), and host interface), a clock generator, and seven buses (three address and four data), making the overall system low cos', low power, and compact.

- Invisible Pipeline: The three-stage instruction pipeline is essentially invisible to the programmer, allowing straightforward program development in either assembly language or a high-level language.

- Instruction Set: The 62 instruction mnemonics are MCU-like, making the transition from programming microprocessor to programming the DSP56000/DSP56001 as easy as possible. The orthogonal syntax supports controlling the parallel execution units. The hardware Do Loop instruction and the repeat instruction make writing straight line code obsolete.

- DSP56000/DSP56001 Compatibility: The DSP56000 is identical to the DSP56001 except for the following features:

- 512-word x 24-bit, on-chip program RAM instead of $3.75 \mathrm{k}$ program ROM. 
-32-wurd $x$ 24-bit bootstrap ROM for loading the program RAM from either a byte wide, memory-mapped ROM or via the host interface.

- Low Power: As a CMOS part, the DSP56000/DSP56001 is inherently very low power; however, three other features can reduce power consumption to exceptionally low levels.

- The WAIT instruction shuts off the clock in the internal processor portion of the DSP56000/DSP56001.

- The STOP instruction halts the internal oscillator.

- Power increases linearly with frequency; thus, reducing the clock frequency reduces power consumption.

Fig. 4.6 is a block diagram of DSP56001 based board PC-56 [23]. The main subsystems are as follows:

1. The DSP56001 processor.

2. External 16k data RAM.

3. Parallel data port that is between the PC and the board PC-56.

4. Single channel analog interface.

5. Interrupt-driven interface to the outside world. 


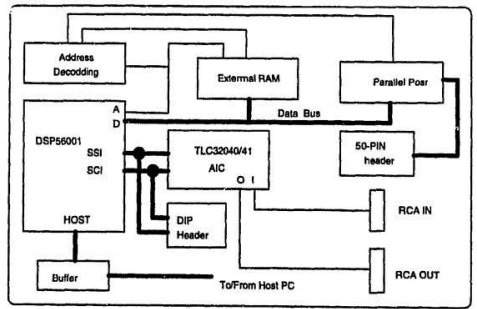

Figure 4.6: PC-56 Block Diagram

\subsection{Ariel's Interface and DSP Library}

A good set of routines for communication of the board with the PC and digital signal processing are provided by Ariel Corporation [24] in a C call-able software library. A brief description of the library is given in the subsequent sections:

\subsubsection{Interface Library}

The PC-56 interface software provides a means to configure and control the PC-56 for user applications through the use of $\mathrm{C}$ call-able routines [24]. The package consists of both 56000 and PC based routines, which when used together allow straightforward control over a user's 56000 application program.

Ariel Monitor(ARIELMON) is the required 56000 monitor. It is a super- 
- forward and inverse FFT,

- magnitude, phase, dB computation (log),

- windowing, scaling, and data acquisition.

Processing of data consists of executing one or more of these commands in a given sequence.

\subsection{Application Software for Spectra Collec- tion}

With the proper use of interface and DSP library, an application program has been developed as part of this work to collect frequency spectra of stator current at different fault conditions. The salient features of algorithm is shown in flowchart form in Fig. 4.7. The detailed program listing is given in Appendix-B and Appendix-C.

\subsection{Experimental Setup}

To acquire different faults related spectra of the stator current of an induction motor following equipment were used:

- Induction Motor.

- Current Probe.

- DSP board 
set of debug monitor(DEGMON), and provides the means for communicating with the $\mathrm{PC}$ in the form of up-load/down-load of program and data memory, as well as user program initialization and termination. It is tied specifically to the $\mathrm{PC}$ routines, which are compatible with Microsoft C V5.1 and Quick C. Other Microsoft languages can use these routines by using the appropriate declarations for setting up the proper $\mathrm{C}$ calling convention.

\subsubsection{DSP-Library}

The FFT Ts $_{56}$ is a software package which enables the PC- 56 or DSP- 56 boards to function as FFT co-processors in an IBM PC/XT/AT or compatible computer. In addition to processing data from the $\mathrm{PC}$, it also supports real time data acquisition using the analog interfaces available on the board.

User has access to the $F F T_{56}$ functions by using low level drivers in conjunction with a set of 56000 programs and data files. The general purpose drivers perform basic functions necessary to control the 56000 and upload/down-load data to the co-processor board.

56000 software consists of a monitor program, which is loaded during the boot cycle, and an application program, loaded by the monitor under control of the PC. The monitor provides the means for communicating with the PC (i.e. up-load/down-load of program and data memory) and is tied specifically to the PC driver routines. The application program consists of a set of FFT related functions which can be initiated by the PC through Host commands. These include: 


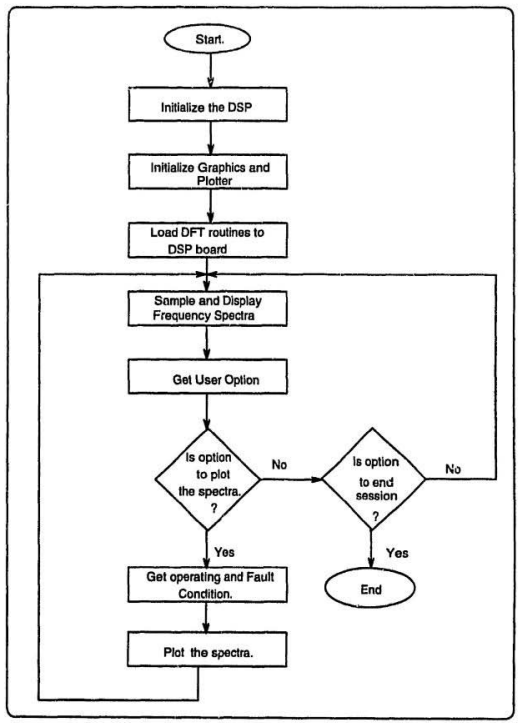

Figure 4.7: Flowchart of the application program 


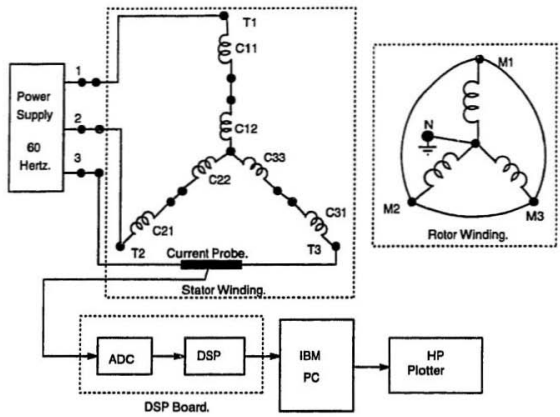

Figure 4.8: Block diagram of the experimental setup.

- IBM PC

- HP-plotter

The experimental setup is shown in Fig. 4.8. In this figure C11, C12, C21, $\mathrm{C} 22, \mathrm{C} 23$ and $\mathrm{C} 33$ are individual coils in the stator winding.

\subsection{Spectra for Different Fault Conditions}

In the laboratory, different types of faults have been occurred in an induction machine. The laboratory wound-rotor induction motor has the following specification:

Specification: 
- Phase: Three

- Voltage: $\frac{120 / 208}{220 / 380}$ volts

- Frequency: $60 / 50 \mathrm{~Hz}$

- Speed: $1725 / 1425$ r.p.m

- Full-load Currents: $\frac{19 / 11}{8.7 / 1.5}$ A

- Horse Power: 2.5/2 H.P.

- Poles : 4

The motor was operated at $208 \mathrm{v}$ and $60 \mathrm{~Hz}$ at no-load condition. Spectra related to different fault currents are shown in the subsequent sections. 


\subsubsection{No Fault condition:}

This is the operating condition which is identical to the set-up as shown in Fig. 4.8. The frequency spectra of the stator current is shown below. Here, in addition to the fundamental component there are also three higher frequencies as the stator current is not perfectly sinusoidal.

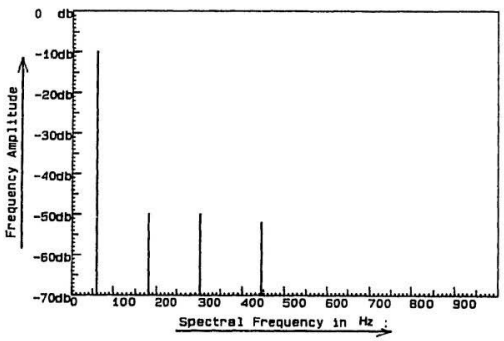

Figure 4.9: Frequency spectra at no fault condition. 


\subsubsection{Stator phase 1 is open:}

Under this operating condition, the phase 1 of the stator has been disconnected from the power supply. The corresponding current spectra is shown below.

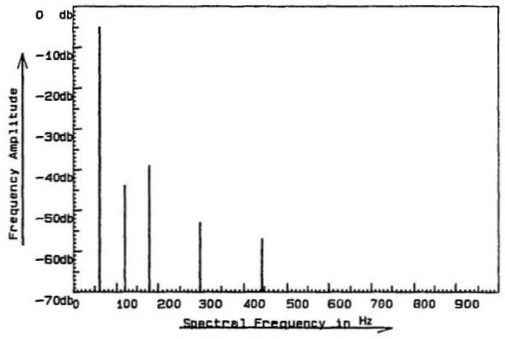

Figure 4.10: Frequency spectra when stator phase 1 is open. 


\subsubsection{Stator phase 2 is open:}

To do it in the Laboratory the phase 2 of the stator was discornected from the power supply. The corresponding current spectra is shown below.

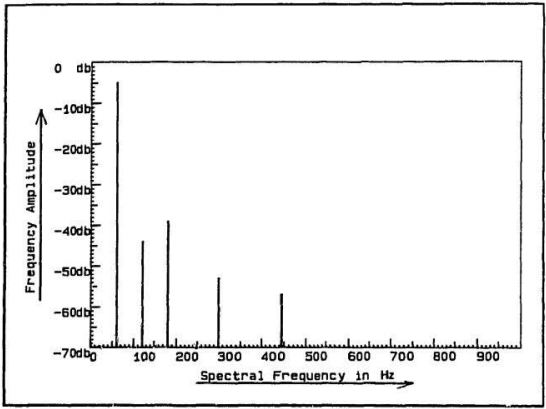

Figure 4.11: Frequency spectra when stator phase 2 is open.

\subsubsection{Stator phase 3 is open:}

Under this condition as current probe is in phase 3 as shown in Fig. 4.8, every frequency component has zero value. 


\subsubsection{Short circuit fault through resistance in Stator Phase 1:}

This is the condition when coil $\mathrm{C} 11$ as shown in Fig. 4.8 was disconnected and a resistor of value $9.5 \mathrm{ohm}$ was connected externally in its position. The corresponding frequency spectra is shown below.

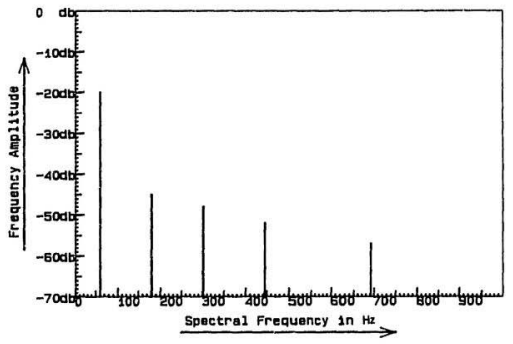

Figure 4.12: Frequency spectra when stator one coil of phase 1. has been externally replaced by a resistor. 


\subsubsection{Short circuit fault through resistance in Stator Phase 2:}

Under this condition coil C21 as shown in Fig. 4.8 was disconnected and a resistor of value $9.5 \mathrm{ohm}$ was connected externally in it's position. The corresponding frequency spectra is shown below.

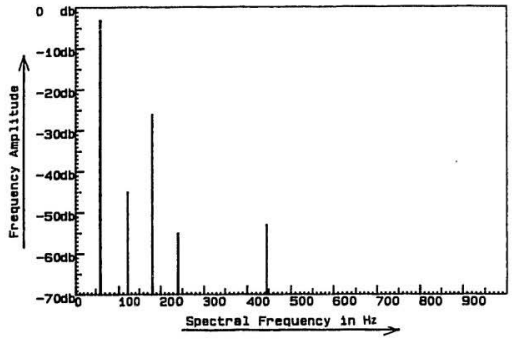

Figure 4.13: Frequency spectra when stator? one coil of phase 2. has been externally replaced by a resistor. 


\subsubsection{Short circuit fault through resistance in Stator Phase 3:}

At this condition coil C31 as shown in Fig. 4.8 was disconnected and a resistor of value $9.5 \mathrm{ohm}$ was connected externally in its position. The corresponding frequency spectra is shown below.

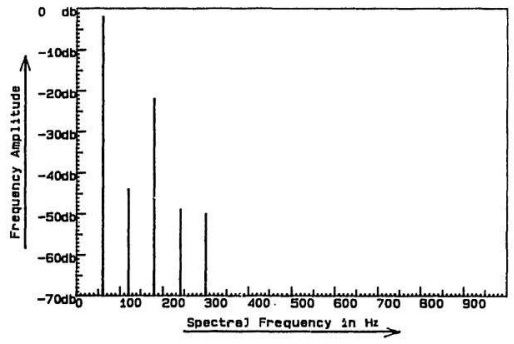

Figure 4.14: Frequency spectra when stator one coil of phase 3. has been externally replaced by a resistor. 


\subsubsection{Short circuit fault in Stator Phase 1:}

This is the condition when coil $\mathrm{Cll}$ as shown in Fig. 4.8 was disconnected and coil $\mathrm{C} 12$ was directly connected to $\mathrm{T} 1$. The frequency spectra under this operating condition is shown below.

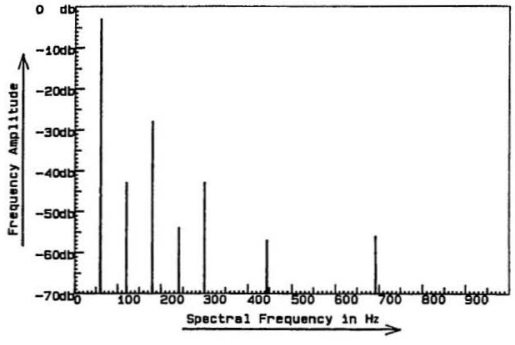

Figure 4.15: Frequency spectra when stator toe coil of phase 1 was shorted. 


\subsubsection{Short circuit fault in Stator Phase 2:}

Under this condition coil C21 as shown in Fig. 4.8 was disconnected and coil C22 was directly connected to terminal T2. The frequency spectra is shown below.

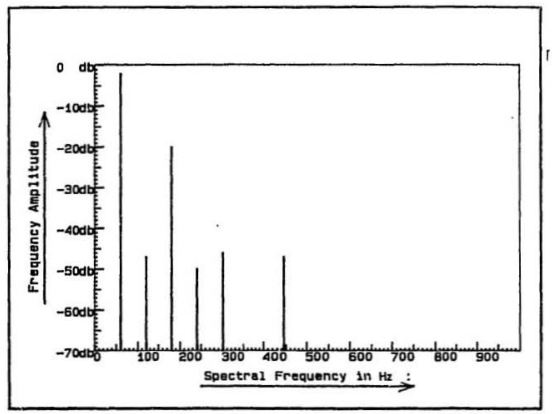

Figure 4.16: Frequency spectra when stator dne coil of phase 2 was shorted. 


\subsubsection{Short circuit fault in Stator Phase 3:}

At is the condition coil C31 as shown in Fig. 4.8 was disconnected and coil C33 was directly connected to terminal T3. The corresponding frequency spectra is snown below.

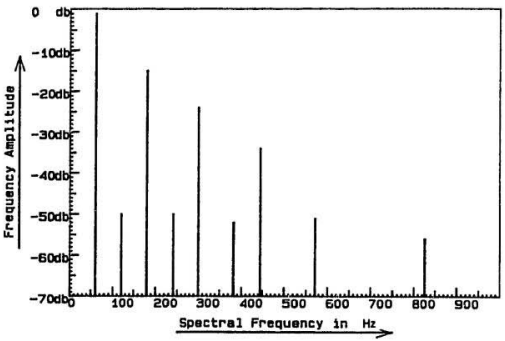

Figure 4.17: Frequency spectra when stator one coil of phase 3 was shorted. 


\subsubsection{Rotor open circuit fault in phase 1:}

According to the Fig. 4.8 the rotor terminals M1,M2 and M3 are short circuited. To make an open circuit fault the rotor's phase M1 was disconnected. Under this condition, the frequency spectra of the stator current is shown below:

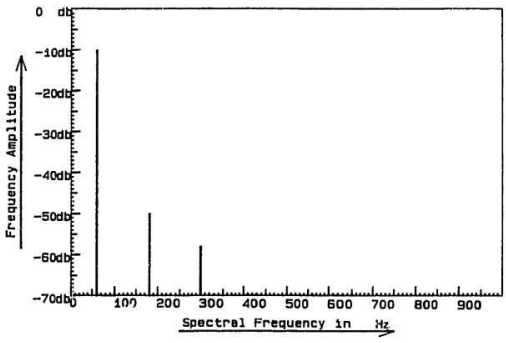

Figure 4.18: Frequency spectra of the stator current when rotor one phase M1 was open circuited. 


\subsubsection{Rotor open circuit fault in phase 2:}

At this condition M2 was disconnected from the close loop of M1, M2 and M3 in rotor's circuit. Under this condition the frequency spectra of the stator current is shown below:

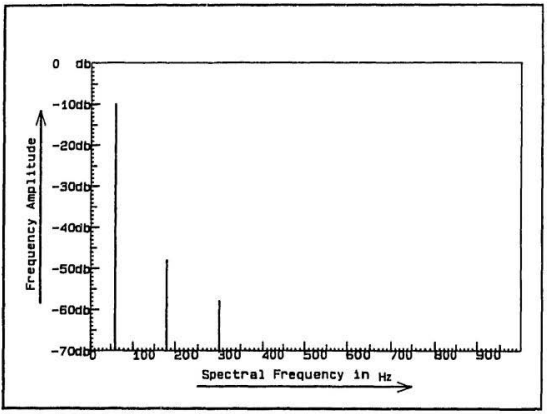

Figure 4.19: Frequency spectra of the stator current when rotor phase M2 was open circuited. 


\subsubsection{Rotor open circuit fault in phase 3:}

To make an open circuit fault in rotor's phase M3, only M1 and M2 were connected and M3 was left open. Under this condition the frequency spectra of the stator current is shown below:

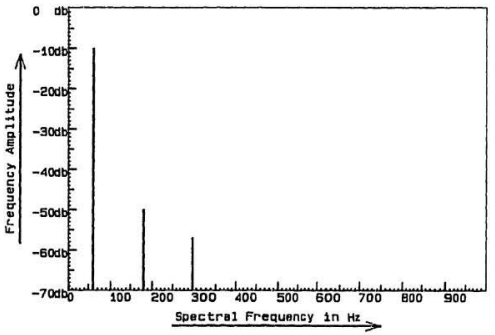

Figure 4.20: Frequency spectra of the stator current when rotor phase M3 was open circuited. 


\subsubsection{Rotor phase 1 is shorted to neural:}

Under the no fault condition, the rotor's terminals M1, M2 and M3 are connected in a single loop as shown in Fig. 4.8. Now to make this fault M1 was disconnected from the loop and it was hooked up to the neutral point of the rotor. It was noted that this type of fault has little impact on wave shape of the stator current whose frequency spectra is shown below:

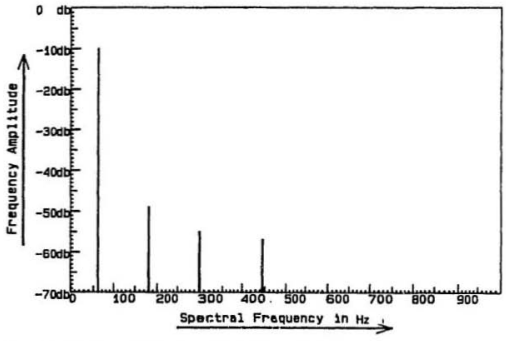

Figure 4.21: Frequency spectra of stator current when rotor phase M1 is shorted to neutral. 


\subsubsection{Rotor phase 2 is shorted to neural:}

At this condition M2 was disconnected form the close loop and it was connected to the neutral point of the rotor. The corresponding frequency spectra is shown in figure below.

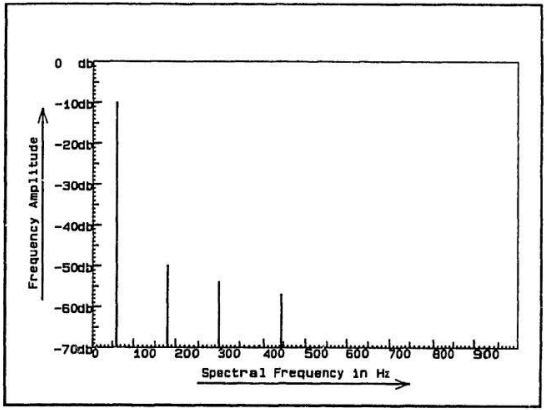

Figure 4.22: Frequency spectra of stator current when rotor phase M2 is shorted to neutral. 


\subsubsection{Rotor phase 3 is shorted to neural:}

Here the rotor terminal M3 was connected to neutral and the terminals M1 and $\mathrm{M} 2$ are shorted. Following figure shows the frequency spectra of stator current under this condition.

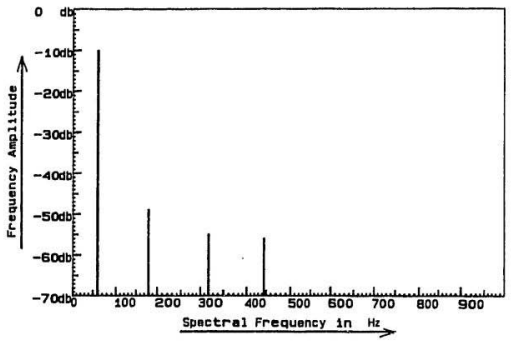

Figure 4.23: Frequency spectra of stator current when rotor phase M3 is shorted to neutral. 


\subsubsection{Rotor short circuit fault through resistance in phase 1:}

Under this situation it has been considered that phase one of rotor was short through resist ance. To make this fault in laboratory M1 was disconnected from the loop as shown in Fig. 4.8 and a resistor of value 6 ohms was connected externally in parallel to M2 and M3. The corresponding frequency spectra is shown below.

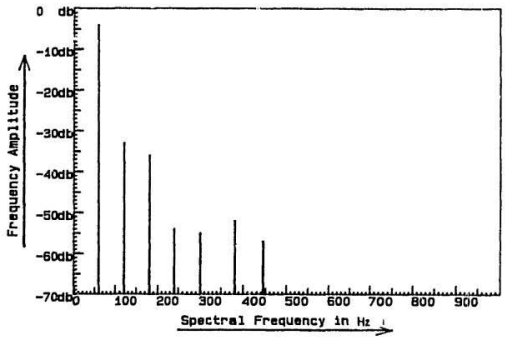

Figure 4.24: Frequency spectra of stator current at rotor short circuit fault through resistance in phase M1. 


\subsubsection{Rotor short circuit fault through resistance in phase 2 :}

M2 was disconnected from the loop and a resistor of value 6 ohms was connected externally in parallel to $M 1$ and M3. The corresponding frequency spectra is shown below.

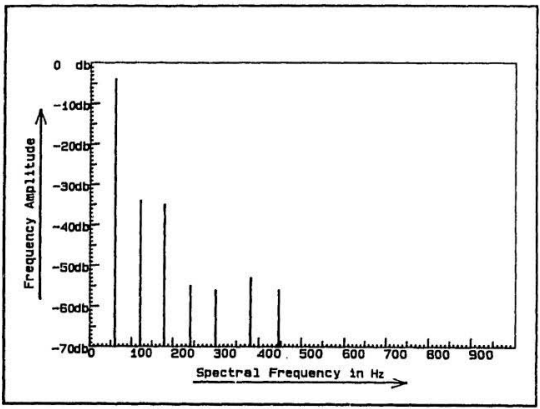

Figure 4.25: Frequency spectra of stator current at rotor short circuit fault through resistance in phase M2 . 


\subsubsection{Rotor short circuit fault through resistance in phase 3 :}

To make this situation in the laboratory M3 was disconnected from the loop and an external resistor of 6 ohms was connected in parallel to M1 and M2. The corresponding frequency spectra is shown below.

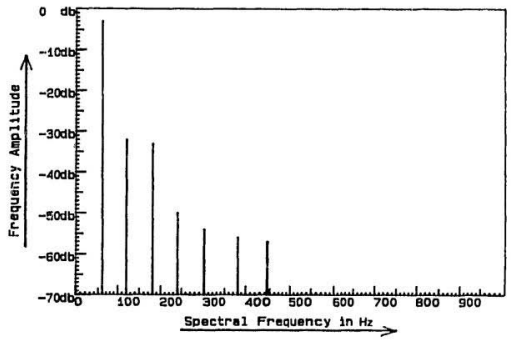

Figure 4.26: Frequency spectra of stator current at rotor short circuit fault through resistance in phase $\mathrm{M} 3$. 


\subsubsection{Rotor phase 1 is unbalanced through an exter- nal resistor:}

In this situation a resistor of 6 ohms was connected in series with the coil Ml without disturbing the close loop situation. The following figure shows the corresponding spectra.

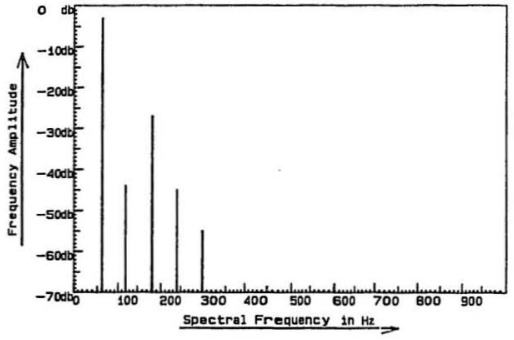

Figure 4.27: Frequency spectra of stator current when there is an unbalance in rotor circuit due to an external resistor in phase M1. 


\subsubsection{Rotor phase 2 is unbalanced through an exter- nal resistor:}

To make it happen in the laboratory a resistor of 6 ohms was connected in series with M2 in the same way as the previous one. The corresponding frequency spectra is shown below.

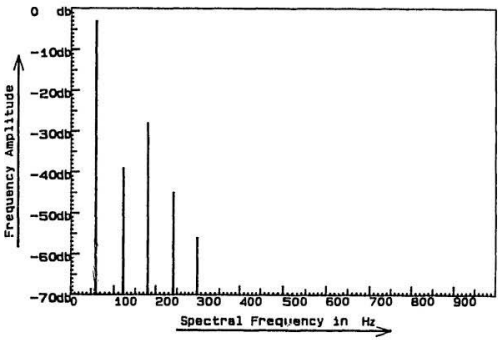

Figure 4.28: Frequency spectra of stator current when there is an unbalance in rotor circuit due to an external resistor in phase M2. 
4.8.22 Rotor phase 3 is unbalanced through an external resistor:

A resistor of 6 ohms was connected in series with M3 . The corresponding spectra is shown below.

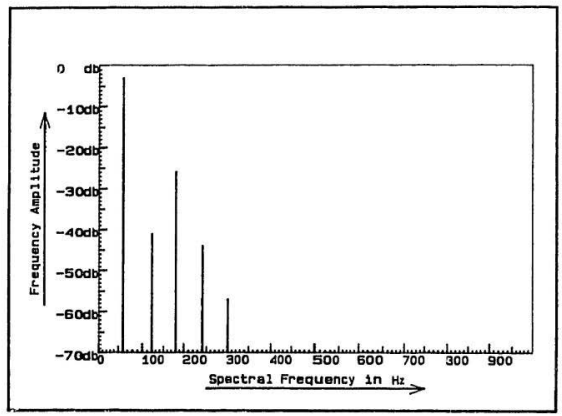

Figure 4.29: Frequency spectra of stator current when there is an unbalance in rotor circuit due to an external resistor in phase M3. 


\subsubsection{Simultaneous open circuit fault in Rotor phase 2 as well as Stator phase 1:}

To make this happen in the laboratory M2 was disconnected from the close loop of the rotor's circuit and phase one of stator was disconnected from the supply. The corresponding frequency spectra of the stator current is shown below.

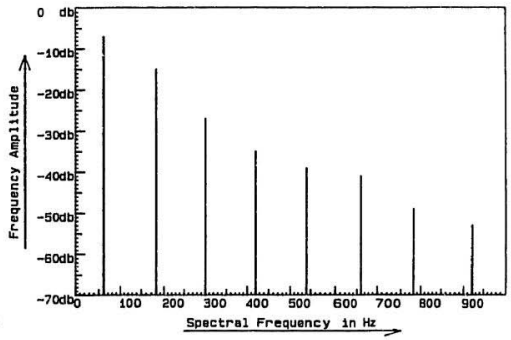

Figure 4.30: Frequency spectra at imultaneous open circuit fault in Rotór. phase 2 as well as Stator phase 1. 


\subsubsection{Simultaneous open circuit fault in Rotor phase 2 as well as Stator phase 2:}

To make this happen in the laboratory M2 was disconnected from the close loop of the rotor's circuit and phase $\mathbf{2}$ of stator was disconnected from the supply. The correquonding frequency spectre is shown below.

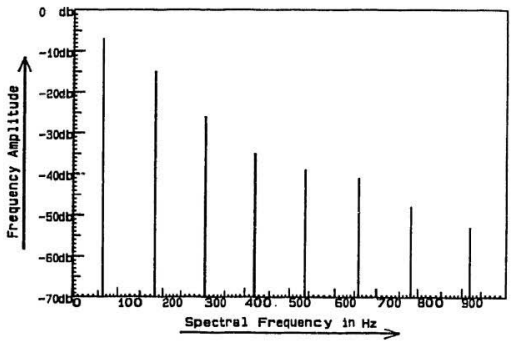

Figure 4.31: Frequency spectra at simultaneous open circuit fault in Rotor * phase 2 as well as Stator phase 1. 


\subsubsection{Simultaneous open circuit fault in Rotor phase 2 as well as Stator phase 3:}

At this condition, as stator's phase 3 was open, so there is no stator current in phase 3 .

\subsubsection{Remarks on Fault Related Frequenency spec- tra of the Stator Current}

Though a sinusoidal voltage was applied to the motor terminals, the frequency spectra of the stator current carries some higher harmonics in addition to the fundamental due to the non-sinusoidal wave shape of the stator current. It should be noted that in the frequency spectra at different fault conditions, frequency components beyond 5 th harmonis are not perfectly divisable by the fundamental; it is due to the slightly non periodic nature of the stator current.

The frequency spectra of the stator current in case of stator's open circuit fault in phase 1 as well as phase $\mathbf{2}$ are almost identical, though they are different from those of other types of faults. So, it is difficult to differentiate stator open circuit fault in phase 1 from that in phase 2 . The same situation is also prevailing in case of rotor open circuit fault and short circuit faults. Moreover, in case of rotor's phase to neutral short circuit fault, the spectra of the stator current is very close to that in case of no fault condition. But the spectra of the major types of faults are significantly different, which opens up the door to use neural network based fault diagnosis scheme. 


\section{Chapter 5}

\section{Fault Recognition by ART2 Neural Network}

\subsection{Introduction}

The performance of ART2 in pattern recognition both in noise free as well as noisy conditions has been reported in chapter 3. This neural network : not only capable of classifying patterns accurately but also can report if it cannot do so. Moreover, it is suitable in dynamic environment like incipient fault diagnosis of induction motor, as it does not require retraining of already trained patterns to adapt itself to new faults. Frequency spectra of stator current at different fault conditions have been reported in chapter 4. As patterns of those spectra are different for different types of faults, pattern recognition technique can be applied on these spectra to diagonise incipient faults of the induction motor. Under this situation, ART2 neural network can be used to diagonise incipient faults of induction motors based on pattern recognition scheme of frequency spectra of the stator current. 
Table 5.1: Table of Faults with unique number.

\begin{tabular}{|c|c|c|}
\hline Serial No. & Pattem No. & Incipiont Faults. \\
\hline 0 & 0 & No Fault \\
\hline 1 & 11 & Open circuit fault in Stator's phase 1 \\
\hline 2 & 12 & Open circuit fault in Stator's phase 2 \\
\hline 3 & 13 & Open clrcult feult in Stator's phase 3 \\
\hline 4 & 21 & Short circult fauth through resiztance in Stator's Phase 1 \\
\hline 5 & 22 & Short circult fauht through resistanco in Stator's Phaso 2 \\
\hline 6 & 23 & Short circuit faull through resistance in Stabrs Phase 3 \\
\hline 7 & 31 & Short circuit fault in Statorts phese 1 \\
\hline 8 & 32 & Short drauit fault in Stators phase 2 \\
\hline 9 & 33 & 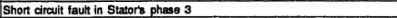 \\
\hline 10 & $\$ 1$ & Open cirzult faut In Rotor's phase 1 \\
\hline 11 & 42 & Open circult faut in Rowors phase 2 \\
\hline 12 & 43 & Open circult fauth in Rotorte phase 3 \\
\hline 13 & 61 & Rotorte Phase 1 is short to neutral \\
\hline 14 & 52 & Rotor's Phase 2 is short to neutral \\
\hline 15 & 53 & Rotors Phase 3 is short to neutral \\
\hline 16 & 61 & Short circuit tault through resiatance in Potor's Phase 1 \\
\hline 17 & 62 & Short circuit fault through resistance in Potor's Phase 2 \\
\hline 18 & 63 & Short circuit fault trough resistancos in Rotor's Phese 3 \\
\hline 10 & 71 & Rotors phase 1 unbelanced by an external weries resistance \\
\hline 20 & 72 & Rotore phase 2 unbelanced by an extomal sories resistance \\
\hline 21 & 73 & Rotorts phase 3 unbelanced by an extomal sories resistance \\
\hline 22 & 81 & Simultanecus open chrcult fault in Rotort phase 2 as wel as Statorts phase 1 \\
\hline 23 & B2 & Simultaneous open circuit fault in Rotor's phase 2 as wel es Stator's phase 2 \\
\hline 24 & 83 & Simulaneous open circuin tault in Rotor's phase 2 as well as Stator's phase 3 \\
\hline
\end{tabular}

\subsection{Training Data Set}

To formulate the fault related spectra in a two-dimensional training data set. it is convenient to give unique number to each fault as shown in Table 5.1. The corresponding values of spectral components have been shown in Table 5.2. In this work eight major classes of faults have been studied. To give unique class numbers to different classes of faults pattern number has been used, where the leftmost character of the pattern number represent the class of faults. No fault condition has been numbered as ' 0 '. 
Table 5.2: Fault related spectral compcnents in matrix form . Frequency in $\mathrm{Hz}$.

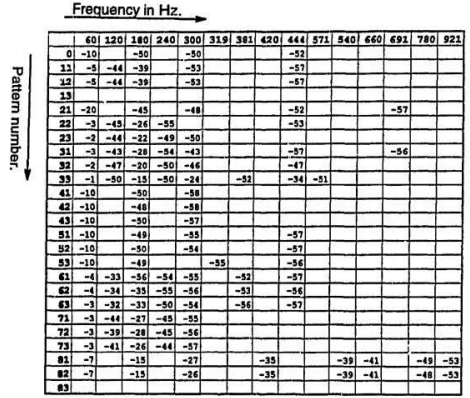

\subsubsection{Data Reduction:}

Now to get a suitable training matrix representing patterns of different farlts, the following processing have been performed on the matrix of Table 5.2, and a.ter these processing a new matrix has been obtained as shown in Table 5.3.

1. All the components having negative values have been deducted from 70 to make them positive as minimum value of a component may be -70 . 
Table 5.3: Training Matrix of fault related current spectra.

Frequency in $\mathrm{Hz}$.

\begin{tabular}{|c|c|c|c|c|c|c|c|c|c|c|}
\hline & 60 & 120 & 180 & 240 & 300 & 319 & 301 & 420 & 44 & 371 \\
\hline 0 & 30.00 & 0.00 & 20.00 & 0.00 & 20.00 & 0.00 & 0.00 & 0.00 & 18.00 & 0.00 \\
\hline 21 & 32.50 & 26.00 & 31.00 & 0.00 & 17.00 & 0.00 & 0.00 & 0.00 & 13.00 & 0.00 \\
\hline 12 & 32.50 & 26.00 & 31.00 & 0.00 & 17.00 & 0.00 & 0.00 & 0.00 & 13.00 & 0.00 \\
\hline 13 & 0.00 & 2.00 & 4.00 & 6.00 & 8.00 & 10.00 & 12.00 & 14.00 & 16.00 & 18.00 \\
\hline 21 & 25.00 & 0.00 & 25.00 & 0.00 & 22.00 & 0.00 & 0.00 & 13.00 & 11.00 & 0.00 \\
\hline 22 & 33.30 & 25.00 & 44.00 & 13.00 & 0.00 & 0.00 & 0.00 & 0.00 & 17.00 & 0.00 \\
\hline 23 & 34.00 & 26.00 & 49.00 & 21.00 & 20.00 & 0.00 & 0,00 & 0.00 & 0.00 & 0,00 \\
\hline 32 & 33.50 & 27.00 & 42.00 & 16.00 & 27.00 & 0.00 & 0.00 & 14.00 & 13,00 & 0.00 \\
\hline 32 & 31.00 & 23.00 & 50,00 & 20.00 & 24.00 & 0.00 & 0.00 & 0.00 & 23.00 & 0.00 \\
\hline 33 & 34.30 & 20.00 & 59.00 & 20.00 & 46.00 & 0.00 & 18.00 & 0.00 & 36.00 & 19.00 \\
\hline 41 & 30.00 & 0.00 & 20.00 & 0.00 & 12.00 & 0.00 & 0.00 & 0.00 & 0.00 & 0.00 \\
\hline 42 & 30.00 & 0.00 & 22.00 & 0.00 & 12.00 & 0.00 & 0,00 & 0.00 & 0.00 & 0.00 \\
\hline 43 & 30.00 & 0.00 & 20.00 & 0.00 & 13.00 & 0.00 & 0.00 & 0.00 & 0.00 & 0.00 \\
\hline 51 & 30.00 & 0.00 & 21.00 & 0.00 & 15.00 & 0.00 & 0.00 & 0.00 & 13.00 & 0.00 \\
\hline 52 & 30.00 & $0.0 \mathrm{~g}$ & 20.00 & 0.00 & 16.00 & 0.00 & 0.00 & 0.00 & 13.00 & 0.00 \\
\hline 53 & 30.00 & 0.00 & 21.00 & 0.00 & 0.00 & 15.00 & 0,00 & 0.00 & 14.00 & 0.00 \\
\hline 61 & 33.00 & 37.00 & 14.00 & 16.00 & 15.00 & 0.00 & 18.00 & 0.00 & 13.00 & 0.00 \\
\hline 62 & 33.00 & 36.00 & 35.00 & 19.00 & 14.00 & 0.00 & 17.40 & 0.00 & 14.00 & 0.00 \\
\hline 63 & 33.30 & 38.00 & 37.00 & 20.00 & 16.00 & 0.00 & 14.00 & 0.00 & 13.00 & 0.00 \\
\hline 72 & 33.50 & 26.00 & 13.00 & 25.00 & 15.00 & 0.00 & 0.00 & 0.00 & 0.00 & 0.00 \\
\hline 72 & 33.50 & 31.00 & 12.00 & 25.00 & 14.00 & 0.00 & 0.00 & 0.00 & 0.00 & 0.00 \\
\hline 73 & 33.50 & 29.00 & 44.00 & 26.00 & 13.00 & 0.00 & 0.00 & 0.00 & 0.00 & 0.00 \\
\hline 21 & 31.50 & 0.00 & 35.00 & 0.00 & 43.00 & 31.00 & 29.00 & 35.00 & 21.00 & 17.00 \\
\hline 82 & 31.50 & 0.00 & 59.00 & 0.00 & 44.00 & 31.00 & 29.00 & 35,00 & 22.00 & 17.00 \\
\hline 03 & 0.00 & 2.00 & 4.00 & 6.00 & 8.00 & 10.00 & 12.00 & 14.00 & 16.00 & 18.00 \\
\hline
\end{tabular}

2. All blanks cells have been filled by zeros to make them sensible to neural network.

3. Spectral components of higher frequencies have been shifted to lower empty spaces as there are only few components at that region.

4. As fundamental component is dominant, so it has been reduced by fifty percent to give importance to other components which really carry fault related information. 
Table 5.4: Faults mapping of the trained network in high precition domain

\begin{tabular}{|c|c|c|}
\hline Beridita. & Pettern Ha. & rochoient Fectis. \\
\hline 0 & 0 & No Fein \\
\hline 1 & 11 & 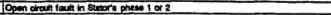 \\
\hline 2 & 12 & Open etran tain in stemore pheses 1 or 2 \\
\hline 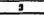 & 13 & 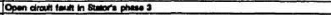 \\
\hline 4 & 21 & 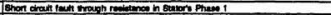 \\
\hline 8 & 22 & 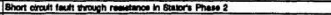 \\
\hline 0 & 23 & 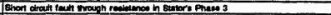 \\
\hline 7 & 31 & Short dirant fain in Btator phaes 1 \\
\hline$B$ & 32 & 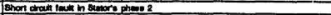 \\
\hline 0 & 33 & Shont cirat teck in siatore pheses 3 \\
\hline 10 & 41 & 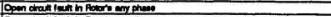 \\
\hline 11 & 42 & Open derat lesa in Potor's ony phose \\
\hline 12 & 43 & 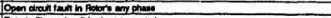 \\
\hline 13 & 81 & 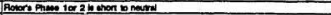 \\
\hline 14 & sh & Aotar Phien 1 or 2 b chort on neutrd \\
\hline 18 & 89 & Rotors Phese 3 is thent io necion \\
\hline 16 & 61 & 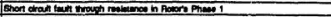 \\
\hline 17 & 62 & 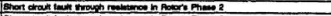 \\
\hline 10 & 62 & 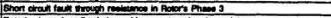 \\
\hline 10 & 71 & 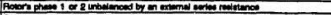 \\
\hline 20 & 72 & 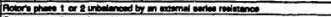 \\
\hline 21 & 73 & 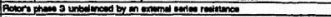 \\
\hline 28 & 81 & 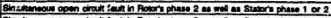 \\
\hline 23 & 22 & 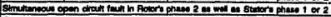 \\
\hline 24 & 83 & Open chain feith in Etetore Phere 3 \\
\hline
\end{tabular}

\subsection{Structure of the Network}

Now the matrix as shown in Table 5.3 represents the training matrix. As this is a $25 \times 10$ dimension matrix, so the cornponents on F1 layer of ART2 should be 10. Now there are 25 different patterns to be recorded in the neural network, but a number of them are similar, so the number of nodes on F2 layer should be less than 25 . For this reason it has been choosen to be 20 . 


\subsection{Training of the Network}

Training of the network has been performed in high precision domain. It should be noted that training time with 25 patterns of dimension 10 as shown in Table 5.3 is very low and it is less than one minute. At the end of the training the knowledge of fault diagnosis is now embedded in the weight vector and the network has classified different patterns as the signature of corresponding faults as shown in Table 5.4. The bottom-up and top-down weight matrices are shown in Table 5.5 and Table 5.6, respectively. From Table 5.4 some important remarks regarding the fault classification of ART2 based on frequency spectra of the stator current, can be noted in the following points:

1. Faults having pattern numbers 11 and 12 have been classified in the same group. As their frequency spectra of the stator current are similar, so the network is unable to differentiate between them.

2. In case of pattern numbers 51 and 52 as frequency spectra are similar, so network has grouped them in the same class. It should be noted that the spectra of pattern numbers 51,52 and 53 have similar shape to that of pattern number $\mathbf{0}$. So, there is a chance that network may recognize rotor's short-circuit-to-neutral fault as no fault condition.

3. The same situation is prevailing in case of pattern numbers 71 and 72 as well as 81 and 82 . In fact in such cases, as the spectra are close to each other, it is difficult for the neural network to differentiate between them. 
Table 5.5: Top-down weight matrix of the trained network.

\begin{tabular}{|l|l|l|l|l|l|l|l|l|l|}
\hline 8.34 & 0.00 & 5.56 & 0.00 & 5.56 & 0.00 & 0.00 & 0.00 & 5.00 & 0.00 \\
\hline 7.24 & 5.79 & 6.90 & 0.00 & 3.79 & 0.00 & 0.00 & 0.00 & 2.89 & 0.00 \\
\hline 0.00 & 0.00 & 0.00 & 0.00 & 3.04 & 3.80 & 4.56 & 5.32 & 6.07 & 6.83 \\
\hline 6.62 & 0.00 & 6.62 & 0.00 & 5.83 & 0.00 & 0.00 & 3.44 & 4.77 & 0.00 \\
\hline 6.46 & 4.82 & 8.49 & 2.89 & 0.00 & 0.00 & 0.00 & 0.00 & 3.28 & 0.00 \\
\hline 6.02 & 4.61 & 8.50 & 3.72 & 3.54 & 0.00 & 0.00 & 0.00 & 0.00 & 0.00 \\
\hline 6.17 & 4.98 & 7.74 & 2.95 & 4.98 & 0.00 & 0.00 & 0.00 & 0.00 & 0.00 \\
\hline 5.63 & 3.81 & 0.29 & 3.31 & 3.98 & 0.00 & 0.00 & 0.00 & 3.81 & 0.00 \\
\hline 4.70 & 2.72 & 7.49 & 2.72 & 6.26 & 0.00 & 0.00 & 0.00 & 4.90 & 0.00 \\
\hline 9.87 & 0.00 & 6.58 & 0.00 & 3.95 & 0.00 & 0.00 & 0.00 & 0.00 & 0.00 \\
\hline 9.00 & 0.00 & 6.30 & 0.00 & 4.50 & 0.00 & 0.00 & 0.00 & 3.90 & 0.00 \\
\hline 8.93 & 0.00 & 6.25 & 0.00 & 0.00 & 4.47 & 0.00 & 0.00 & 4.17 & 0.00 \\
\hline 6.85 & 7.68 & 2.91 & 3.32 & 3.11 & 0.00 & 3.74 & 0.00 & 2.70 & 0.00 \\
\hline 6.14 & 6.70 & 6.51 & 2.79 & 2.60 & 0.00 & 3.16 & 0.00 & 2.60 & 0.00 \\
\hline 6.18 & 7.01 & 6.83 & 3.69 & 2.95 & 0.00 & 0.00 & 0.00 & 0.00 & 0.00 \\
\hline 6.13 & 5.67 & 7.66 & 4.57 & 2.56 & 0.00 & 0.00 & 0.00 & 0.00 & 0.00 \\
\hline 6.19 & 5.36 & 8.13 & 4.80 & 0.00 & 0.00 & 0.00 & 0.00 & 0.00 & 0.00 \\
\hline 4.08 & 0.00 & 7.12 & 0.00 & 5.56 & 4.01 & 3.75 & 4.53 & 2.72 & 0.00 \\
\hline 0.00 & 0.00 & 0.00 & 0.00 & 0.00 & 0.00 & 0.00 & 0.00 & 0.00 & 0.00 \\
\hline 0.00 & 0.00 & 0.00 & 0.00 & 0.00 & 0.00 & 0.00 & 0.00 & 0.00 & 0.00 \\
\hline
\end{tabular}

Table 5.6: Bottom-up weight matrix of the trained network.

\begin{tabular}{|l|l|l|l|l|l|l|l|l|l|l|l|l|l|l|l|l|l|l|l|l|l|}
\hline 0.34 & 7.24 & 0.00 & 6.62 & 6.46 & 6.02 & 6.17 & 5.63 & 4.70 & 9.87 & 9.00 & 6.93 & 6.65 & 6.14 & 6.18 & 6.13 & 6.19 & 4.08 & 0.00 & 0.00 \\
\hline
\end{tabular} \begin{tabular}{|l|l|l|l|l|l|l|l|l|l|l|l|l|l|l|l|l|l|l|l|l|l|l|l|}
\hline 0.00 & 5.79 & 0.00 & 0.00 & 1.62 & 4.61 & 4.90 & 3.61 & 2.72 & 0.00 & 0.00 & 0.00 & 7.68 & 6.70 & 7.01 & 5.67 & 5.36 & 0.00 & 0.00 & 0.00 \\
\hline 5.06
\end{tabular} \begin{tabular}{|l|l|l|l|l|l|l|l|l|l|l|l|l|l|l|l|l|l|l|l|l|l|}
\hline 5.56 & 6.90 & 0.00 & 6.62 & 8.49 & 0.50 & 7.74 & 0.29 & 7.49 & 6.50 & 6.30 & 6.25 & 2.91 & 6.51 & 6.83 & 7.68 & 8.13 & 7.12 & 0.00 & 0.00 \\
\hline
\end{tabular} \begin{tabular}{|l|l|l|l|l|l|l|l|l|l|l|l|l|l|l|l|l|l|l|l|l|l|l|l|l|l|l|}
\hline 0.00 & 0.00 & 0.00 & 0.00 & 2.89 & 3.72 & 2.95 & 3.31 & 2.72 & 0.00 & 0.00 & 0.00 & 3.32 & 2.79 & 3.69 & 4.57 & 1.60 & 0.00 & 0.00 & 0.00 \\
\hline
\end{tabular} \begin{tabular}{|l|l|l|l|l|l|l|l|l|l|l|l|l|l|l|l|l|l|l|l|l|l|l|l|}
\hline 5.56 & 3.79 & 3.04 & 5.93 & 0.00 & 3.54 & 4.99 & 3.98 & 6.26 & 3.95 & 4.50 & 0.00 & 3.11 & 2.60 & 2.95 & 2.56 & 0.00 & 5.56 & 0.00 & 0.00 \\
\hline
\end{tabular}

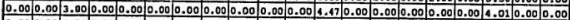

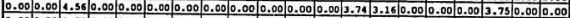

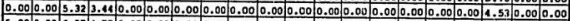
\begin{tabular}{|l|l|l|l|l|l|l|l|l|l|l|l|l|l|l|l|l|l|l|l|}
\hline 5.00 & 2.89 & 6.07 & 4.77 & 3.28 & 0.00 & 0.00 & 3.81 & 4.90 & 0.00 & 3.90 & 4.17 & 2.70 & 2.60 & 0.00 & 0.00 & 0.00 & 2.72 & 0.00 & 0.00 \\
\hline
\end{tabular}

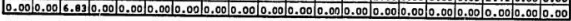




\subsection{Fault Recognition by the trained net- work.}

To test the diagnostic performance of the trained network both in noise free as well as noisy conditions, the training data set was taken as first test pattern and after that noisy data set. The performance of the network to diagnose the fault in noise free condition is identical to that of Table 5.4. To simulate a noisy situation, random noise was added to spectral components as shown in Table 5.8. Every bordered cells including shaded cells are corrupted, but shaded ones are corrupted heavily. Now the trained network has been asked to diagnose the faults related to these noisy spectra and the diagnostic performance is reported in Table 5.7. From this table it should be noted that the trained network is unable to detect three faults in this noisy condition. This operational scenario can be improved to handle higher noise margin, but that will loose accuracy by training the network in low precision mode. Under this situation the pattern mapping in training stage and fault diagnosis performance in previous noisy situation are shown in Table 5.9 and Table 5.10 , respectively. Table 5.10 aparently shows better performance with only one detection failure but Table 5.9 shows that the network has mapped the rotor short-circuit-to-neutral fault as no fault condition. Thus there exists a need for optimization of accuracy and noise margin. It is beyond the scope of this thesis. 


\subsection{Model of ART2 neural network based Incipient Fault Detection System}

Based on the performance of ART2 neural network in fault diagnosis of induction motor using the frequency spectra of the stator current, it is evident that the basic requirements for incipient fault detection of induction motors can be met with ART2 neural network based system. Fig. 5.1 gives the schematic of such a fault detection system. Here the controller gives the frequency spectra of the stator current to the trained network which detects the present internal condition of the machine. If it can diagnose a fault, the controller gets specific fault related information from the corresponding database and can report to the user. If the neural network is unable to detect a fault, the controller asks the user for information related to the specific situation and simultaneously updates its knowledge-base as well as the underlying neural network. 
Table 5.7: Fault related noisy current spectra.

\begin{tabular}{|c|c|c|c|c|c|c|c|c|c|c|}
\hline & 60 & 120 & 110 & 240 & 300 & 319 & 391 & 120 & 404 & 571 \\
\hline 0 & 30.00 & 0.00 & 20.00 & 0.00 & 20.00 & 0.00 & 0.00 & 0.00 & 18.00 & 0.00 \\
\hline 21 & 32.30 & 26.00 & 32.00 & 0.00 & 17.00 & 2.00 & 0.00 & 0.00 & 23.00 & 0.00 \\
\hline 12 & & & 30.00 & 0.00 & & & & & & 0.00 \\
\hline 13 & .00 & .00 & 1.00 & 6.00 & 8.00 & 10.00 & 12.00 & .00 & 16.00 & 18.00 \\
\hline 22 & & .00 & 25.00 & 00 & 32.00 & & 0.00 & & 9.00 & 0.00 \\
\hline 22. & .50 & .00 & 30.00 & 15.00 & & 00 & 0.00 & 00 & 0.00 & 0.00 \\
\hline 23 & 34.00 & 25.00 & 48.00 & & 20.00 & 0.00 & 0.00 & 0.00 & 0.00 & 0.00 \\
\hline 31 & 33.50 & .00 & 12.00 & 18.00 & 27.00 & & 0.00 & 14.00 & 13.00 & 0.00 \\
\hline 32 & 34.00 & 23.00 & 52.00 & 21.00 & 24.0 & 0. & 0.00 & & 23.00 & 0.00 \\
\hline 39 & & 20.00 & 35. & 20.00 & & & 18.00 & & 3.00 & 19.00 \\
\hline 4 & .00 & 00 & 20.00 & 0.00 & 12.00 & & 00 & & .00 & 0.00 \\
\hline 42 & .00 & 0.00 & inetis: & 0.00 & & 0.00 & 0.00 & .00 & 0.00 & 0.00 \\
\hline 69 & 30.00 & 0.00 & 20.00 & 0.00 & 13.00 & 0.00 & 0.00 & & 0.00 & 0.00 \\
\hline 51 & & 0.00 & 22.00 & 0.00 & 15.00 & 0.00 & 0.00 & & & 0.00 \\
\hline 52 & 30.00 & 0.00 & 20.00 & 0.00 & 16.00 & & 0.00 & & & 0.00 \\
\hline 53 & .00 & .00 & 21.00 & 0.00 & 0.00 & 15.00 & 0.00 & .00 & & 0.00 \\
\hline 61 & .00 & .00 & 11,00 & 16.00 & 15.00 & & 18.00 & & 13.00 & 0.00 \\
\hline 69 & 33.00 & 10.00 & 35.00 & 3.00 & & 0.00 & 19.00 & & & 0.00 \\
\hline cas & 33.50 & 38.00 & & 20.00 & 16.00 & 0.00 & 14.00 & 0.00 & 33.00 & 0.00 \\
\hline nI & 33.50 & 26.00 & 43.00 & 25.00 & 15.00 & 0.00 & 0.00 & 0.00 & 0.00 & 0.00 \\
\hline 72 & 33.90 & 31.00 & 12,00 & 25.00 & 13,00 & 0.00 & 0.00 & 0.00 & 0.00 & 0.00 \\
\hline 73 & & 20.00 & 31.00 & 26.00 & 13.00 & 0.00 & 0.00 & & 0.00 & 0.00 \\
\hline 81 & & & & & & 31.00 & 29.00 & 35.00 & 21.08 & 27.00 \\
\hline 12 & 31.50 & & 33.00 & & 44.00 & 31.00 & 29.00 & 35.00 & 22.00 & 17.00 \\
\hline & 0.00 & 2.00 & 1.00 & 6.00 & 0.00 & 10.00 & 12.00 & 14.00 & 16.00 & 18.00 \\
\hline
\end{tabular}

Table 5.8: Diagnostic test result of the trained network in noisy situation.

\begin{tabular}{|c|c|c|}
\hline Gentide. & Puban Na. & incipient Fales. \\
\hline 0 & 0 & Wofeh \\
\hline 1 & 11 & 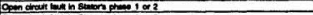 \\
\hline 2 & 12 & 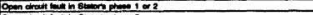 \\
\hline 3 & 18 & 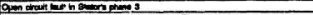 \\
\hline 4 & 21 & 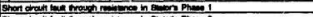 \\
\hline 8 & 82 & 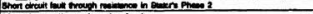 \\
\hline 6 & 23 & Unable to diagonise the fault \\
\hline 7 & 91 & 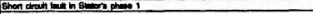 \\
\hline : & 32 & 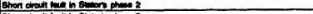 \\
\hline 0 & 33 & ghan want the in 8 and phes 3 \\
\hline 10 & 41 & Open drail heil h how encthen \\
\hline 11 & 42 & Unable to diegonisa the fault \\
\hline 12 & 43 & 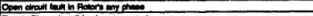 \\
\hline 18 & 81 & 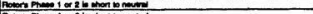 \\
\hline I4 & 82 & 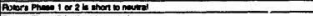 \\
\hline 18 & कs & 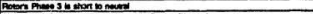 \\
\hline 16 & e1 & 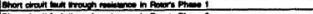 \\
\hline 17 & 92 & 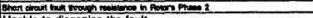 \\
\hline 18 & 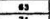 & Unable to dlagenise the fault \\
\hline 10 & 71 & 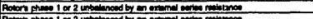 \\
\hline 20 & 72 & 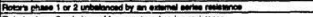 \\
\hline 21 & 73 & 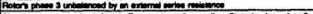 \\
\hline 32 & 1 & 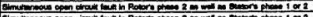 \\
\hline 23 & 12 & 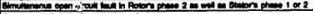 \\
\hline 24 & क & 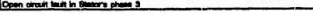 \\
\hline
\end{tabular}


Table 5.9: Faults mapping in training phase in low precision domain.

\begin{tabular}{|c|c|c|}
\hline Eend va. & Powim Ha. & Incipient fater. \\
\hline 0 & 0 & Ho finet \\
\hline 1 & 11 & 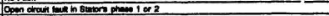 \\
\hline 2 & 12 & 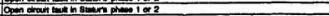 \\
\hline 3 & 13 & 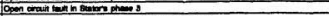 \\
\hline 4 & 21 & 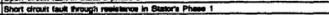 \\
\hline E & 22 & 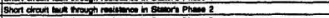 \\
\hline 8 & 23 & 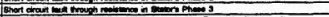 \\
\hline 7 & 31 & Short circedt tautt through vacigtance in Stator's Phase 3 \\
\hline 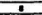 & 32 & 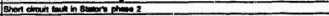 \\
\hline 0 & 93 & 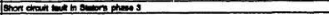 \\
\hline 10 & 41 & 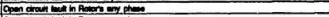 \\
\hline 11 & 42 & 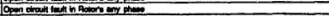 \\
\hline 12 & 43 & 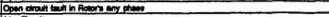 \\
\hline 13 & 61 & Do Fault \\
\hline 14 & 82 & Mo Fault \\
\hline I & E3 & 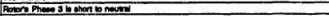 \\
\hline 18 & 81 & 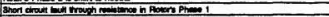 \\
\hline 17 & 82 & 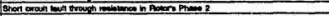 \\
\hline 18 & 6 & Potor's phase 1 or 2 univalenced by en extemal serias resistance \\
\hline 19 & 71 & 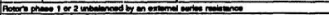 \\
\hline 20 & 72 & 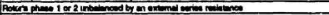 \\
\hline 21 & 73 & 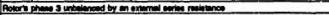 \\
\hline 22 & ei & 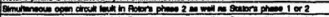 \\
\hline 29 & 12 & 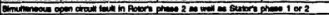 \\
\hline 24 & a & 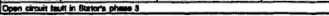 \\
\hline
\end{tabular}

Table 5.10: Fault diagnostic performance of the network trained in low precision domain in noisy situation .

\begin{tabular}{|c|c|c|}
\hline Endin. & Periom No. & 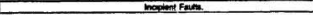 \\
\hline 0 & 0 & Wo Fan \\
\hline 1 & 11 & 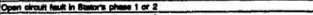 \\
\hline 2 & II & 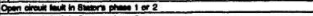 \\
\hline 3 & 19 & 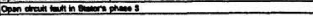 \\
\hline 4 & 21 & 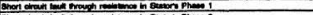 \\
\hline 8 & 22 & 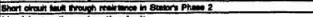 \\
\hline 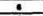 & 23 & Unable to digenges the faull \\
\hline 7 & S1 & 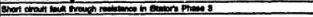 \\
\hline e & 92 & 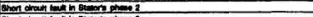 \\
\hline 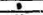 & 33 & 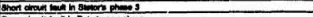 \\
\hline 10 & 41 & 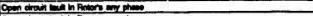 \\
\hline 11 & 42 & 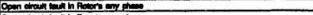 \\
\hline 12 & A3 & 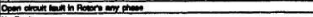 \\
\hline 12 & E1 & Wo kal \\
\hline 14 & 82 & Wo fen \\
\hline 18 & B. & 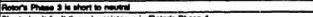 \\
\hline II & OI & 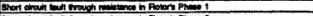 \\
\hline 17 & 12 & 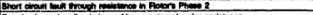 \\
\hline 10. & Q3. & 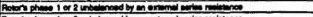 \\
\hline 10 & 71 & 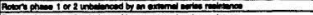 \\
\hline 20 & 72 & 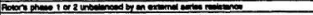 \\
\hline 21 & 73 & 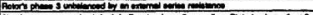 \\
\hline 22 & 61 & 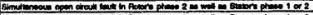 \\
\hline 23 & 12 & 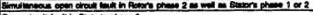 \\
\hline 24 & s3. & 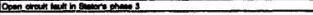 \\
\hline
\end{tabular}




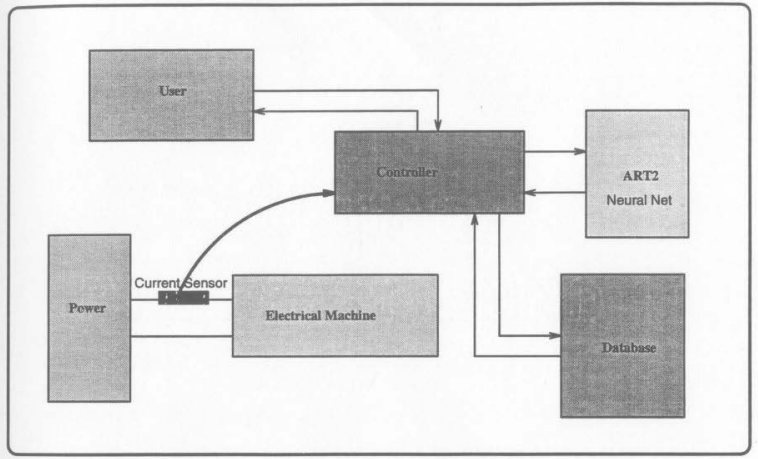

Figure 5.1: Model of ART2 neural network based on-line incipient fault diagnosis system for induction motors. 


\section{Chapter 6}

\section{Conclusions and Recommendations for Future Work}

\subsection{Conclusions}

In this thesis an incipient fault detection scheme of induction motors based on ART2 neural network has been developed. This fault diagnosis scheme is not only capable of detecting a fault but also can report if it cannot diagnose a particular fault, so that preventive steps can be taken to update the underlying neural network to cope with this undetected fault while retaining the already acquired knowledge without retraining of the trained patterns. The accuracy of this fault diagnosis scheme is susceptable to noise margins. For lower noise level, it gives high accuracy in its performance.

A laboratory experimental set-up based on DSP techniques to collect the fault related frequency spectra in real-time of the stator current of induction 
motor has been developed. This is a general purpose frequency spectra collection system, which can be used for other purposes. Frequency spectra of the stator current of a wound-rotor induction motor at different fault conditions making unbalance in the stator as well as rotor circuits have been collected. From the patterns of the spectra it is evident that the major faults can be detected from these spectra through a pattern recognition scheme. But it is difficult to differentiate similar faults as they have identical frequency spectra.

ART2 neural network has been implemented using object oriented soft. ware methodology. Its training, recalling and dynamic updating performance have been studied. It's training time is very low in comparison to the popular Feedforward Neural Network. It can update its knowledge to cope with the new pattern while retaining the acquired knowledge without the need of retraining.

In this thesis work, only stator current has been considered as fault related information carrier. But in order to develop a comprehensive fault diagnosis system, multi-sensors based scheme may give better result. This is beyond the scope of this thesis. Through this research work the present state-ofthe-art of incipient fault detection of an induction motor has been improved to find better neural network and to collect fault related frequency spectra of the stator current, which can be used as a basis for the development of robust and comprehensive fault diagnosis system for electrical machines . 


\subsection{Recommendations for Future Work}

From previous work [25] it is evident that information from a single sensor is not good enough to identify all possible faults that may occur in induction machines. As for example, stator current usually does not carry the signature of the bearing faults. Therefore it is necessary to pay attention in multiple sensors based scheme. Performance of a machine fault diagnosis systen: should be independent of operating conditions of the machine as well as the design parameter variations. Moreover, the system should report not only the type of faults but also the extent of the detected fault. To make it cost-effective and user-friendly for the maintenance engineer in the case of a large manufacturing plant, a single system should be capable to diagonise faults of multiple machines.

The future work should also consider both the theoretical as well as the experimental study to select the optimum number of sensors necessary to collect all major faults related information. For example, a vibration sensor may be necessary for bearing faults.

Data from multiple sensors should be reduced through neural network based filter to avoid redundant information.

Usually faults related information are contaminated by the interference of operating conditions as well as the design parameter variation of the machine. It is thus necessary to develop suitable neural network based filter to get noise free fault related information.

As the extent of a particular fault is fuzzy in nature, therefore, in such a 
case the application of fuzzy logic should be explored. A neuro-fuzzy computing model should be developed for the incipient fault diagnosis of electrical machines. 


\section{Bibliography}

[1] T.S. Sankar," Diagnosis and Monitoring for AC Drives," IAS Conf. record, vol. 1, 1992, pp. 370-377.

[2] Peter J. Tavner, James Penman, Condition Monitoring of Electrical Machines,Research Press. Ltd. John Wiley \& Sons Inc.

[3] Mo-Yuen Chow and Sui-Oi Yee, "Methodology for On-line Incipient Fault Detection in Single Phase Squirrel-case Induction Motors using Artificial Neural Networks, "IBEE Transaction on Energy Conversion, vol. 6,no. 3, Sept. 1991, pp. 536-545.

[4] Mo-yuen Chow, Robert N. Sharpe and James C. Hung, "On the Application and Design Consideration of Artificial Neural Network Fault Detectors-Part II," IEEE Transaction on Industrial Electronics, vol. 40, no.2, April, 1993,pp. 189-196.

[5] Mo-Yuen Chow, Griff Bilbro and Sui Oi Yee, "Application of Learning Theory to an Artificial Neural Network that Detects Incipient Faults in Single Phase Induction Motors, ${ }^{n}$ International Journal of Neural System, vol.2, No.1\&2, 1991, pp. 91-100. 
[6] Chin-Teng and C.S. George Lee, "Neural-network based Fuzzy Logic Control and Decision System," IEEE Trans. on Computers, vol. 40, No. 12, March 1991, pp. 1320-1335.

[7] Timo Sorsa and Heikki N.Koivo, "Neural Network in Process Fault Diagnosis, ${ }^{n}$ IEEE Trans. on System, Man, and Cybernetics, vol. 21, no. 4, March 1991, pp. 815-825.

[8] Petri A. Jokinen, "Comparison of Neural Network Models for Process Fault Detection and Diagnosis Problems," IJCNN Seatle'91, vol. 1, pp. 239-244.

[9] Paul V. Goode and Mo-yuen Chow, "Neural/Fuzzy Systems for Incipient Fault Detection in Induction Motors," IECON'93 vol.1,pp.332-337.

[10] Mo-Yuen Chow, Peter M. Mangum and Sui Ou Yee , A Neural Network approach to Real-time Condition Monitoring of Induction Motors," IEEE Transaction on Industrial Electronics, vol. 38, no. 6, Dec. 1991, pp. 448-453.

[11] Mo-yuen Chow, Robert N. Sharpe and James C. Hung, "On the Application and Design Consideration of Artificial Neural Network Fault Detectors-Part I," IEEE Thansaction on Industrial Electronics, vol. 40, no.2, April, 1993, pp. 181-188.

[12] M.F. Abdel Mageed, A.F. Sakr, A.Bahgat, "Fault Detection and Identification using Hierarchical Neural Network-based System," IECON'98 vol. 1, pp. 338-342. 
[13] F. Filippetti and M.Martelli, "Neural Networks Aided On-line Diagnostics of Induction Motor Rotor Faults, ${ }^{n}$ IAS Conf. record, 1993, pp. 316323.

[14] F. Filippetti and M.Martelli, "Development of Expert System Knowledge-base to On-line Diagnosis of Rotor Electrical Faults of Induction Motors," IAS Conf. record, 1992, pp. 92-99.

[15] R. Natarajan, "Failure Identification of Induction Motors by Sensing Unbalanched Stator Currents ," IEEE Transaction on Energy Conversion, vol. 4, no.4, December, 1989 , pp. 585-590.

[16] C.A. Protopapas, S.D. Kaminaris, AV. Machias and B.C. Papadias, "An Expert System for Fault Repairing and Maintenance of Electraical Machines. " IEEE Transaction on Energy Conversion, vol. 5, no.1, March, 1990, pp. $79-83$.

[17] Simon Haykin,Neural Networks : A Comprehenssive Foundation, Macmillan College Publishing Company, USA, 1994.

[18] James A.Freeman and David M.Skapura,Neural Networks Algorithm, Application and Training Techniques, Addition-Welsy Publishing Company, 1991.

[19] Gail A.Carpenter and Stephen Grossberg, "Invarient pattern recognition and recail by an attentive self-organizing ART architecture in a nonstationary world," Proceedings of the IEEE First International Conference on Neural Networks, vol. II, June 1987, pp. 737-745. 
[20] Gail A.Carpenter and Stephen Grossberg, "ART2: Self-organization of stable category recognition codes for analog input patterns, ${ }^{n}$ Procecdings of the IEEE First International Conference on Neural Networks, vol. II, June 1987, pp. 727-735.

[21] Alan V.Oppenhein and Ronald W.Schafer,Discrete-Time Signal Processing, Prentice-Hall,Inc,New Jersey 07632,1989.

[22] DSP5600/DSP56001-Dsigital signal Processor : User's Manual, Motorola, 1990.

[23] Operating Manual for the PC-56 : DSP Co-processor Board, Ariel Corporation, 1989.

[24] DSP Developer's Toolkit for the Motorola DSP56000/DSP56001, Ariel Corporation, 1989.

[25] Toshio Fukuda, Koji Shimojima, "Multi-Sensor Integration System with Fuzzy Interference and Neural Networks," International Joint Conference on Neural networks, vol. 11, 1992, pp. 757-762. 


\section{APPENDIX 'A' Program listing for ART2 Neural Network.}

This is the program for implementation of ART2 neural network. Art2 has been implemented using object oriented software development methodology. So it can be used as a class library to add its feature to application program. Dynamic memory operation has been used to handle all matrices, so virtually the size of input pattern is determined by the memory of the system. It also includes an user interface routine to train and test a neural network. It has been written in Borland C++ under O/S MS-DOS for IBM PC environment.

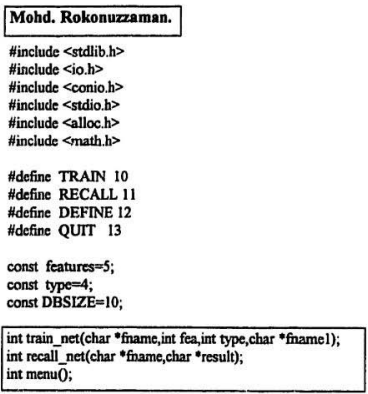




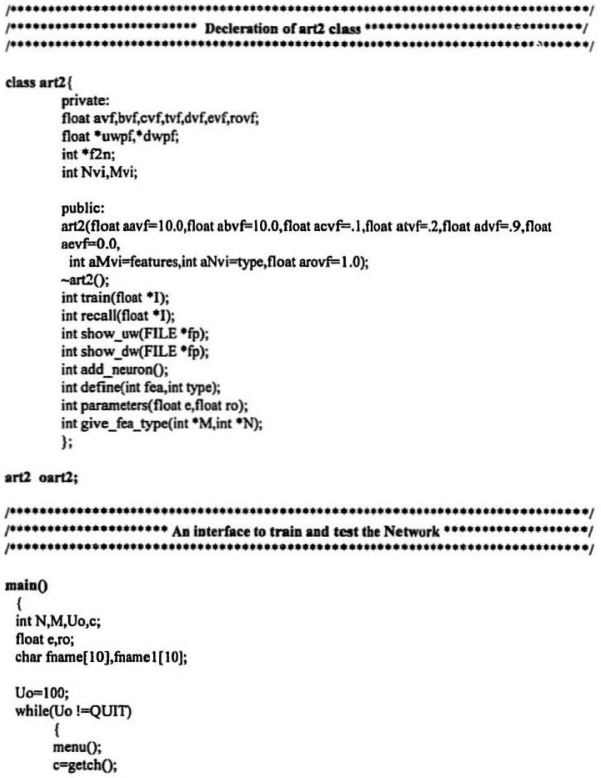




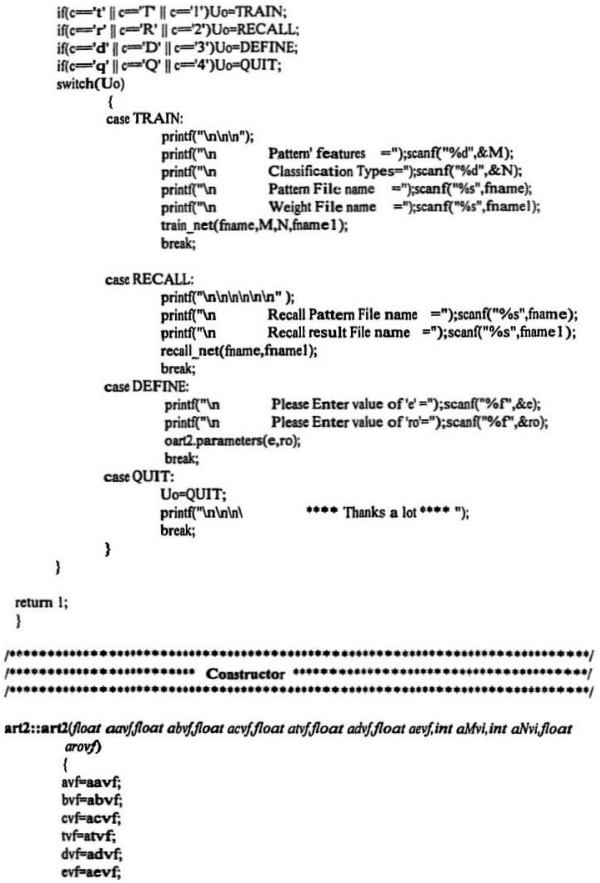




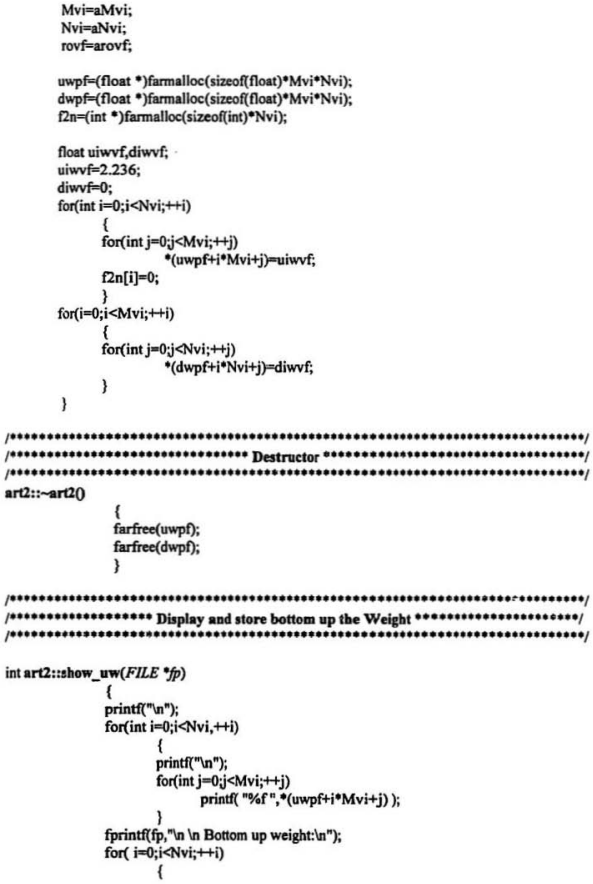




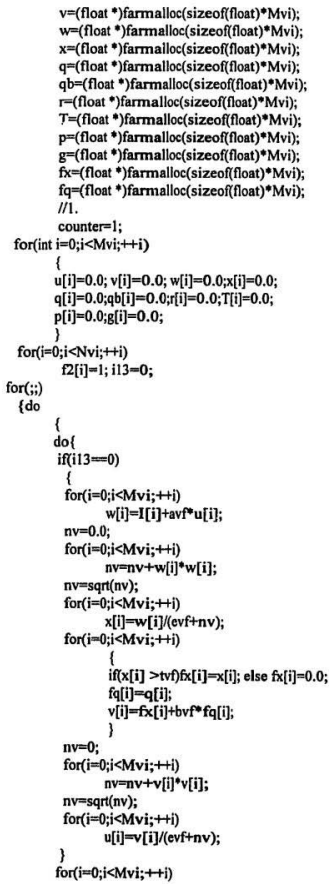




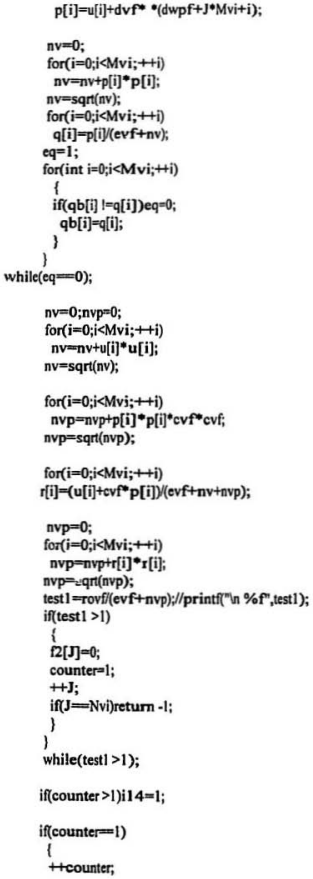




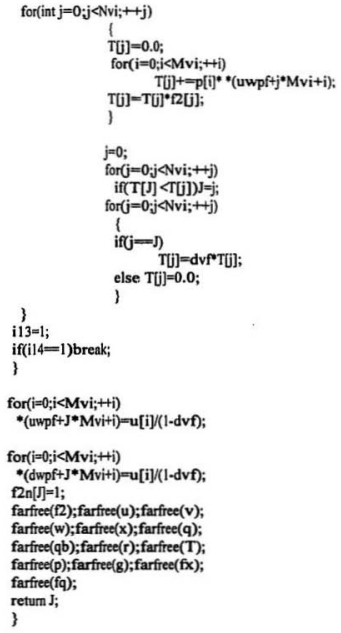




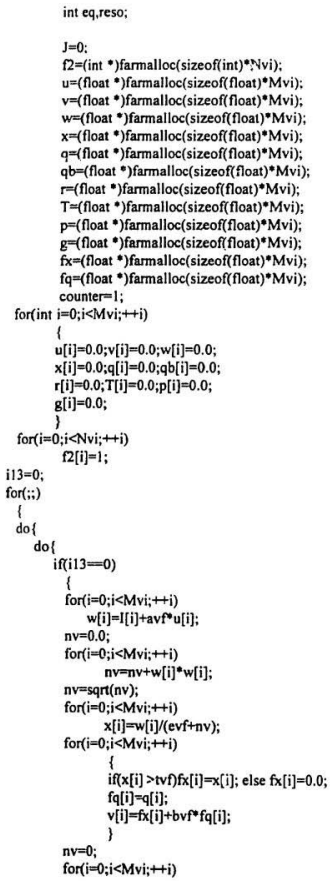




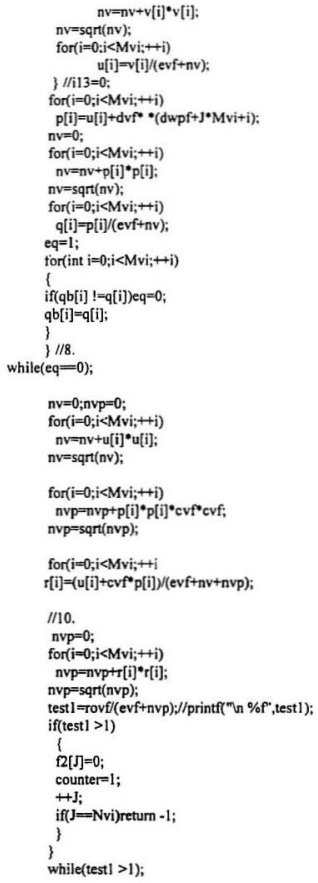


uwpf $\left.=(\text { float *)farmalloc(sizeof(float) })^{*} \mathrm{Mvi}^{*} \mathrm{Nvi}\right)$;

dwpf $=($ float *)farmalloc(sizeof(float)* Mvi* Nvi);

$\mathrm{f} 2 \mathrm{n}=$ (int *)farmalloc(sizeof(int)* Nvi);

float uiwvf,diwvf;

uiwvf=2.236;

$\operatorname{diwvf}=0$;

for(int $i=0 ; i<N v i ;++i)$

1

for(int $\mathrm{j}=0$; j $<$ Mvi; $++j)$

for $(i=0 ; i<M v i ;++i)$

*(uwpf+i*Mvi+j)=uiwvf;

\{

for(int $\mathbf{j}=0 ; \mathrm{j}<\mathrm{N} v i ;++\mathrm{j})$

(dwpf $\left.+\mathrm{i}^{*} \mathrm{Nvi}+\mathrm{j}\right)=\mathrm{diwvf}$

\}

return 1;

\}

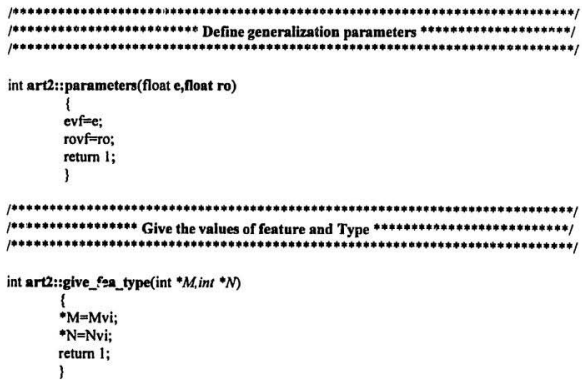




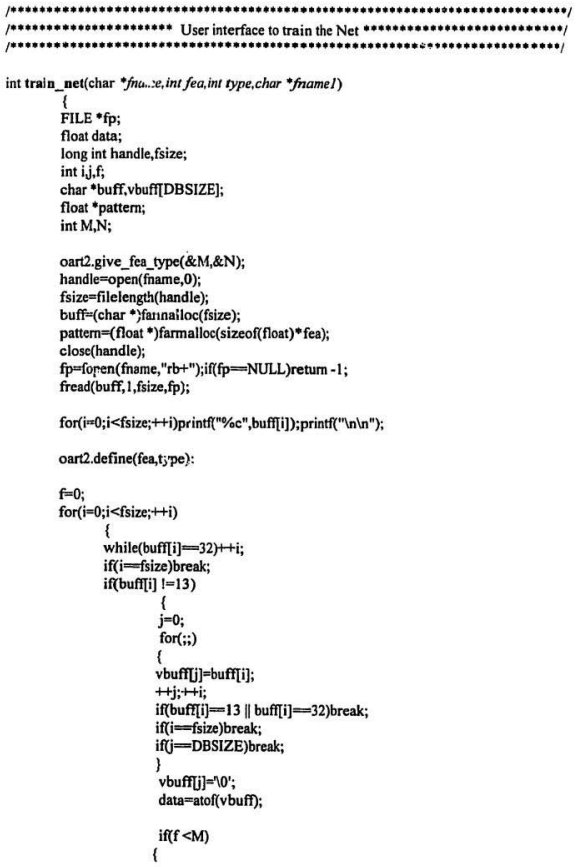




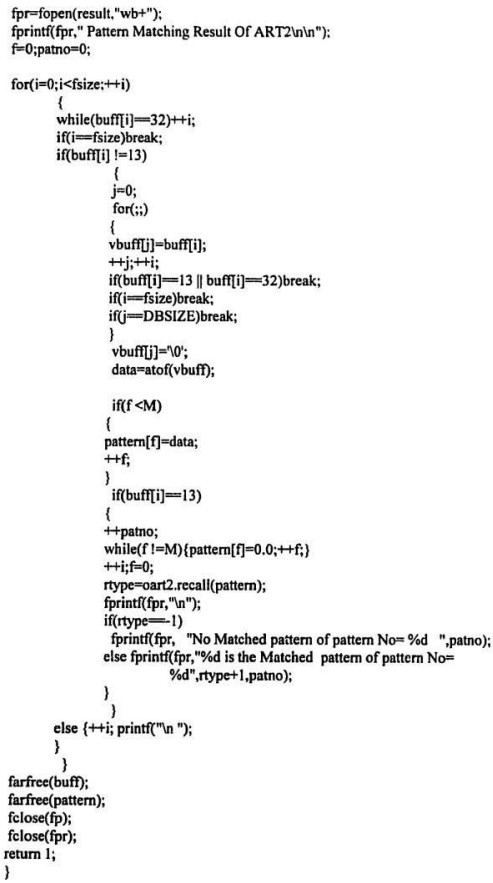




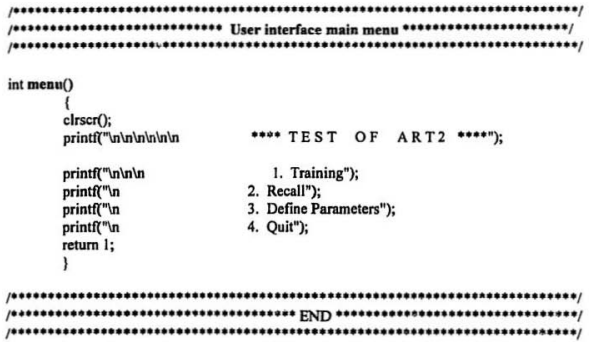




\section{APPENDIX “B" Program listing for real-time frequency spectra acquisition..}

This program is for real-time frequency spectra collection of the stator current of an induction motor using digital signal processing(DSP) technique.

\#define MAX_COM 80

\#include <stallib.h>

\#include <stdio.h>

\#include <conio.h>

\#include <ctype.h>

\#include $\langle$ dos.h>

\#include <graph.h>

\#include <math.h>

\#include "pc56.h" $\quad / 4$ header fie for global pe56 declarations *

\#include "pe56ext.h" /* extemal variable definitions */

/* defined in PC-56 library (init 56.c) */

\#include "fft_56.h" $/ *$ fft_56 definitions */

\#include "demogrph.h" /* graphics definitions */

\#include "printer.h"

\#include "plotter.h"

extem unsigned long int DATA; $\quad 1^{\circ}$ data array

extem unsigned long int COEF; $\quad$; sin/cos table

extern unsigned long int WINDCOF, $\quad$ * window coefficients *I

extem long int $\max N_{i}$

/

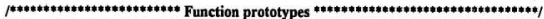

|

void initgrph(long int);

void demoplot(int $\mathrm{D}$, int $\mathrm{D}$, int, int, int);

void pause(void);

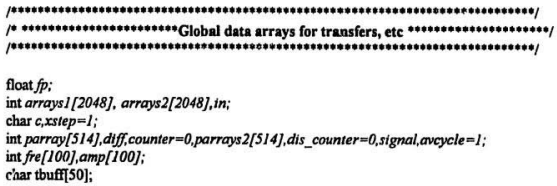

float $f p$;

int arrays1 [2048], arrays2[2048],in;

char $c, x s t e p=1$;

int parray [514], diff,counter $=0$, parrays $2[5] 4]$, dis_counter $=0$, signal, avcycle $=1$;

int fre [100], amp [100];

chas tbuff50]: 


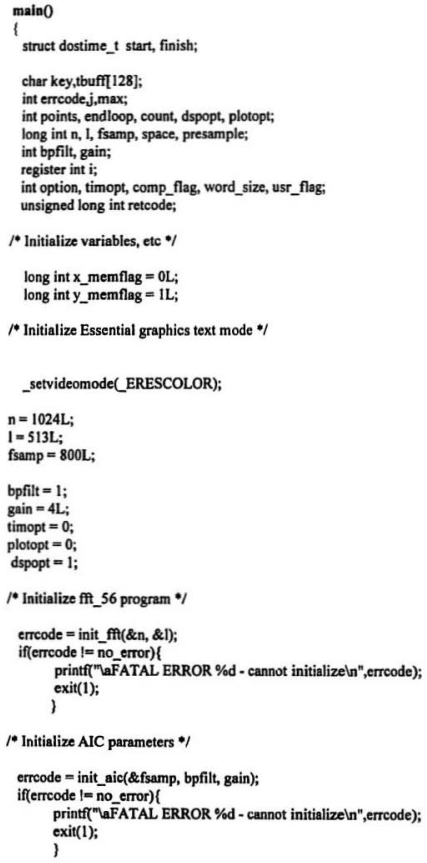




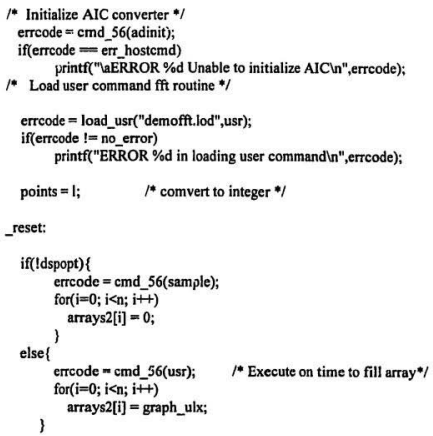

/* Sample and display until keyboard is hit */

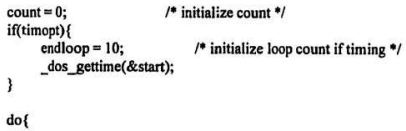

/* Start process, display arrays1, erase arrays $2 *$

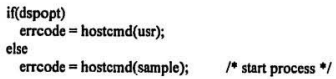

$/ * 1$ demoplot(arrays 1,arrays2,points,yellow,plotopt); */

/* When complete, download into arrays1 */

while(!dsp_done(0)\{ $/ *$ wait for 56000 code to be finished $\%$ 
\}

rcv_hp(\&retcode);

erreode = retcode; $\quad / *$ conversion to int $* /$

if(errcode ! = no_error)

exit(1);

errcode = dwnarras(arrays2, 1, X_SPACE, DATA);

1* Start process for next array, display arrays 2 , erase arrays 1 */

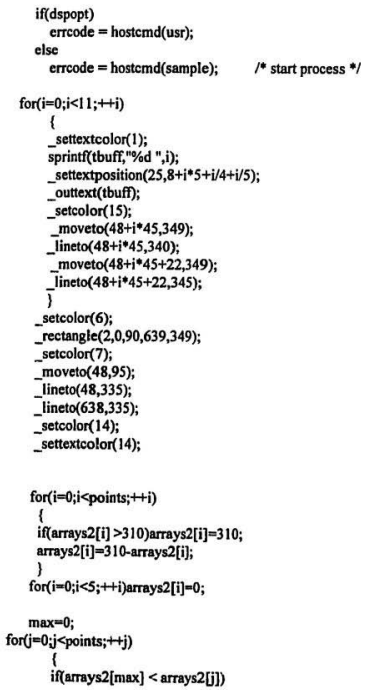




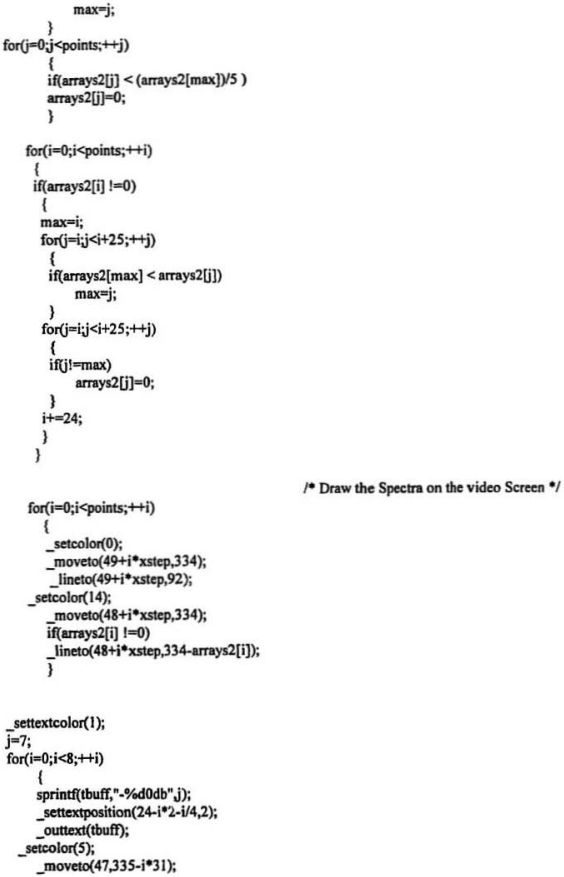

1* Draw the Spectra on the video Screen * 


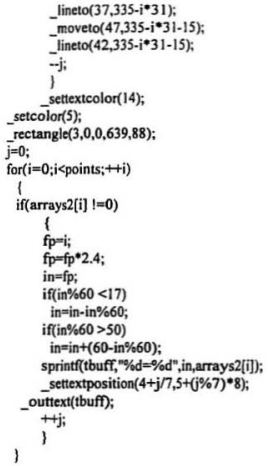




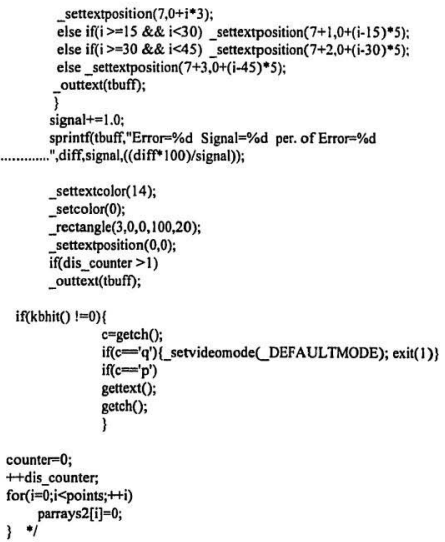

1 When complete, download into arrays1 (at top of loop) */

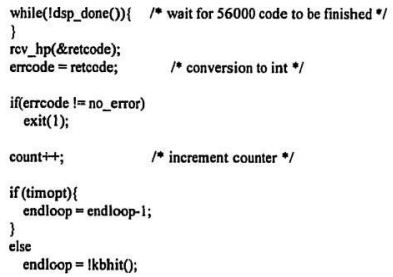




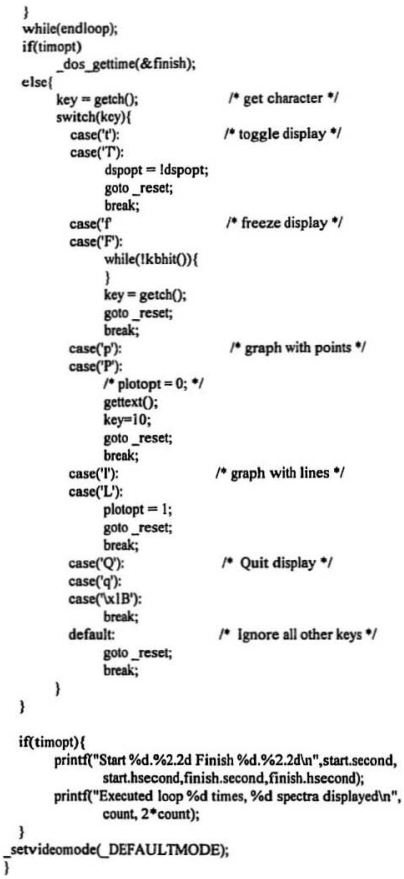




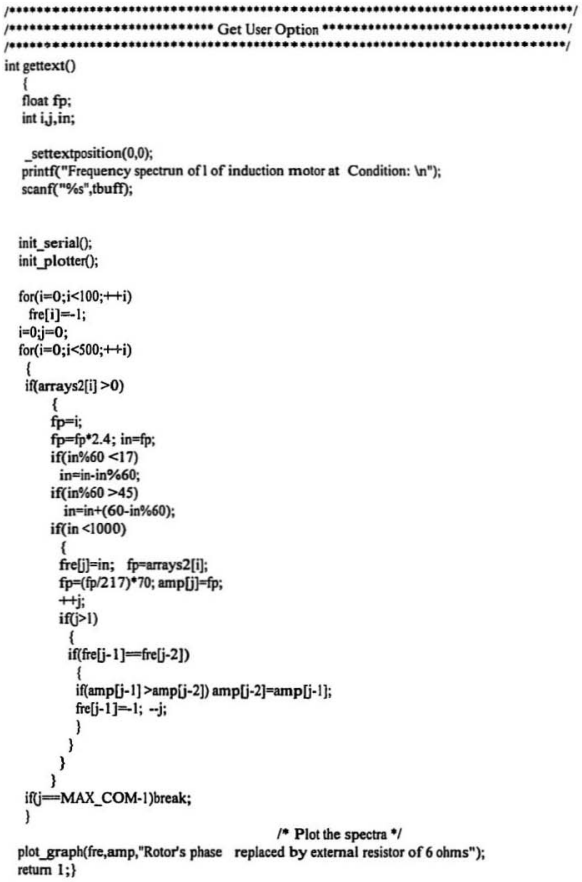




\section{APPENDIX “C” Program listing of HP-plotter driver.}

This is the program for Six pen HP-plotter driver to get real-time plot of frequency spectra of the stator current of an induction motor..

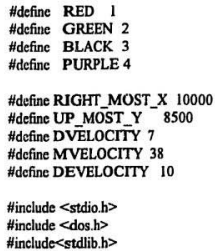




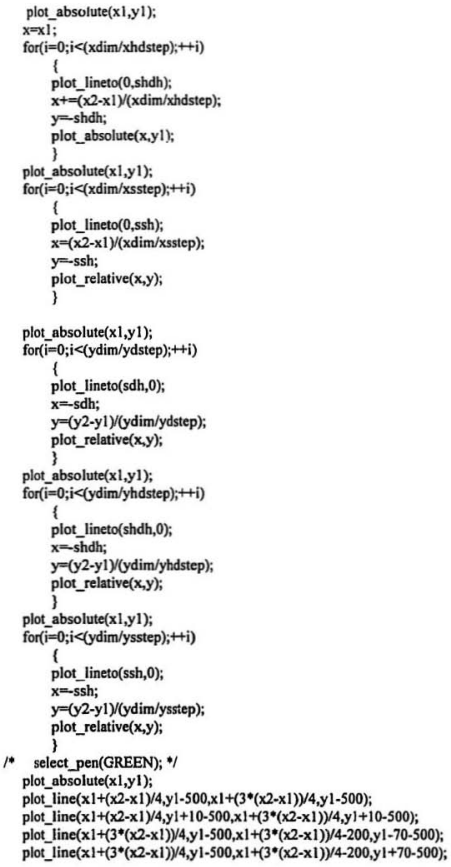




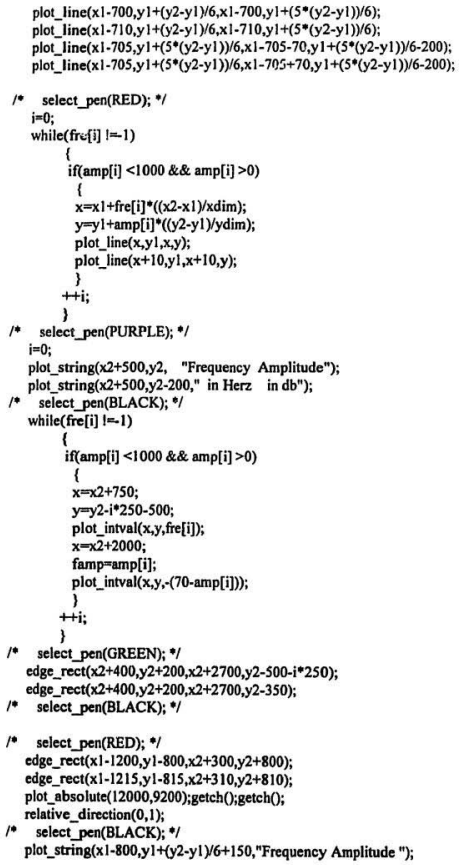




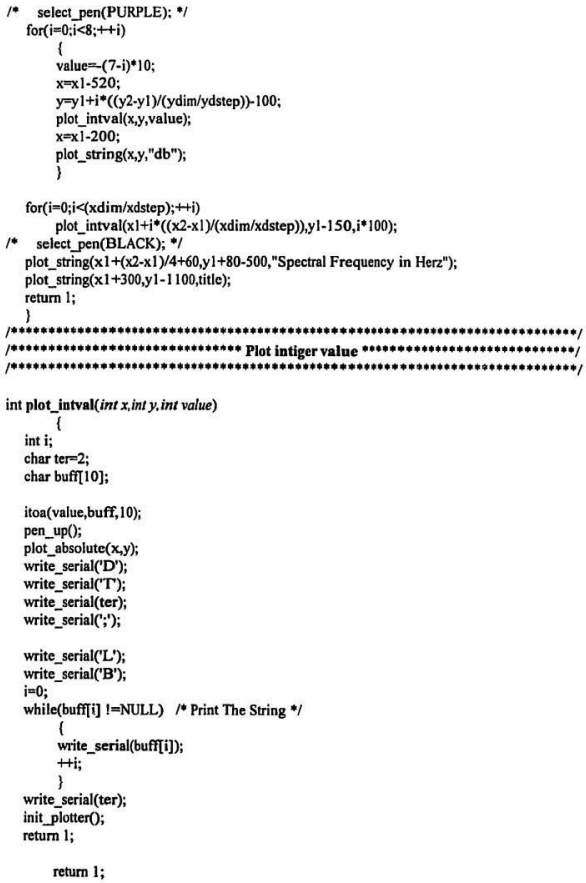




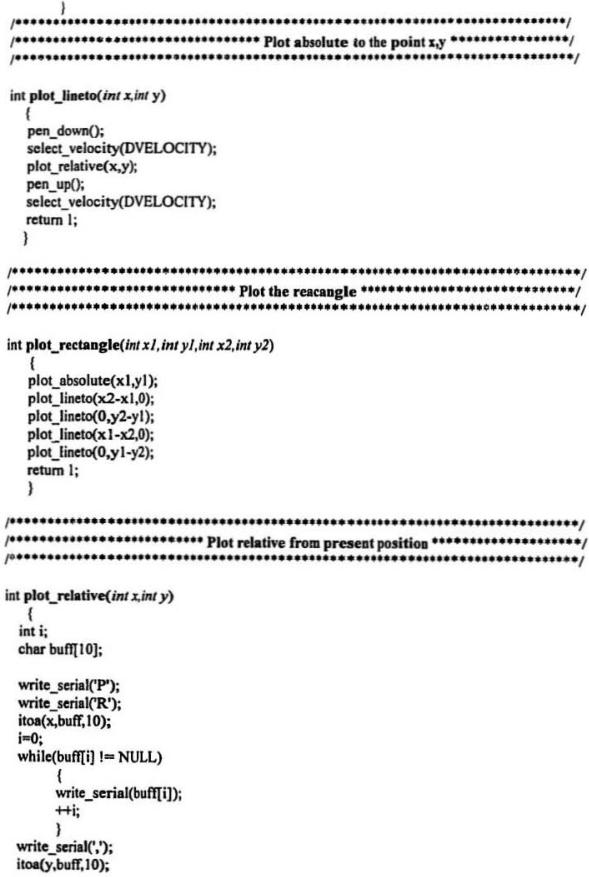




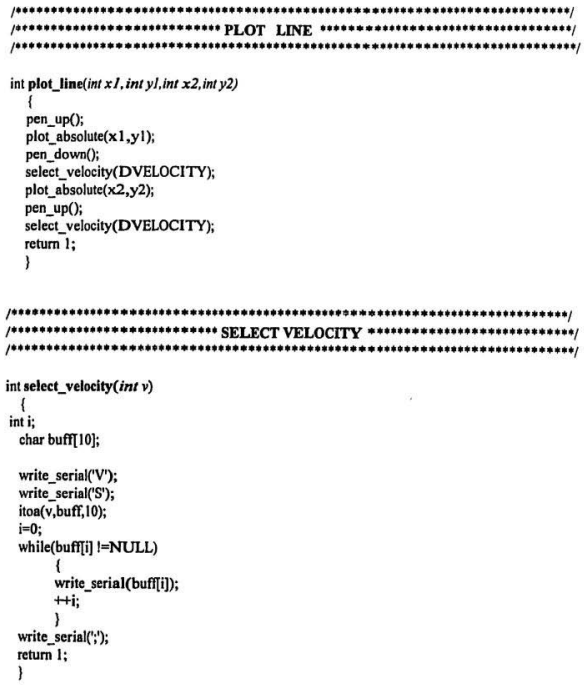




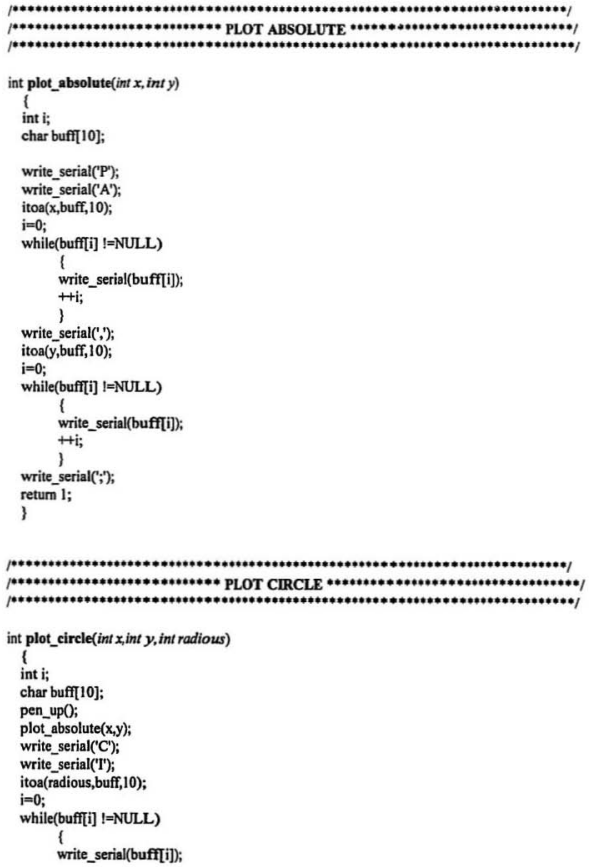




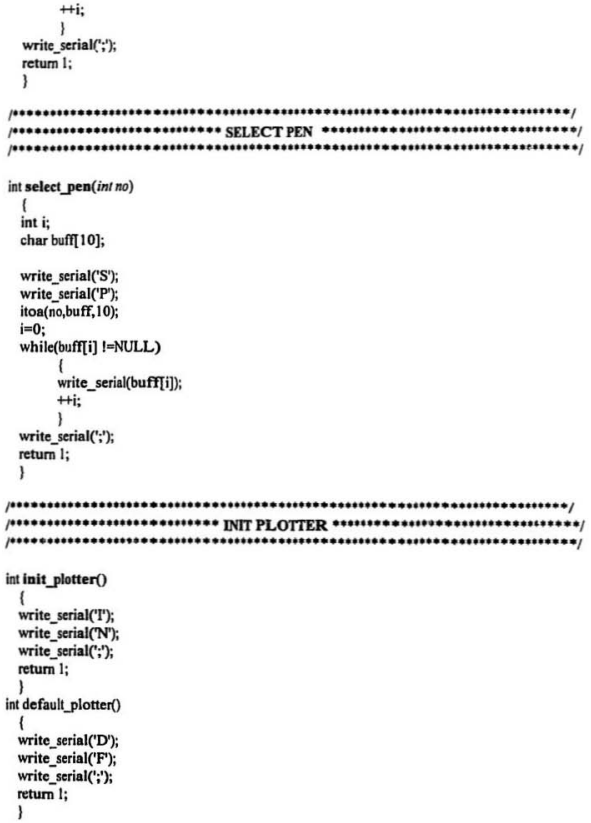




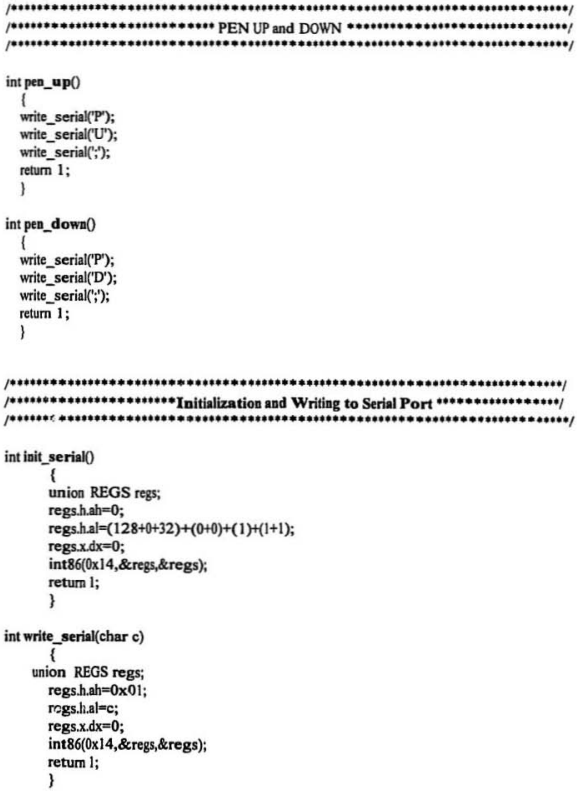




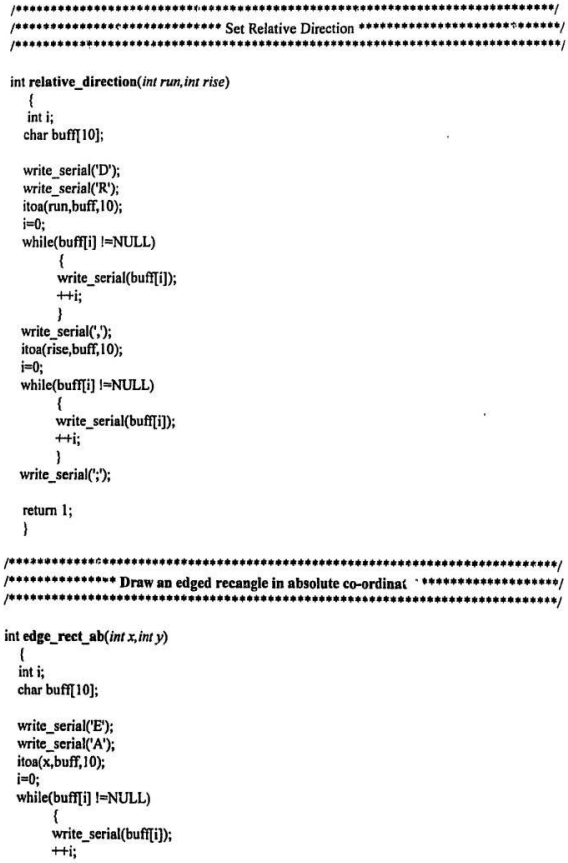


\}

write_serial(',');

itoa( $y$, buff, 10$)$;

$\mathrm{i}=0$;

while(buff[i] !=NULL)

\&

write_serial(buff[i]);

$+\mathrm{i}$;

\}

write_serial(';');

return 1;

\}

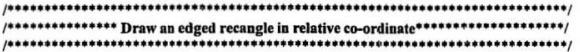

int edge_rect(int $x l_{1}$ int $y 1$, int $x 2$, int $y^{\text {? })}$

\{

int $\mathrm{i}$;

char buff[10];

plot_absolute(xl,y1);

write_serial('E');

write serial('R');

itoa((x2-x1), buff, 10);

$\mathrm{i}=0$;

while(buffi] !=NULL)

\{

write_serial(buff[i]);

$++\mathrm{i}$;

)

write_serial(',");

itoa((y2-y1),buff,10);

$\mathrm{i}=0$;

while(buff[i] I=NULL)

\{

write_serial(buff[i]);

$+\mathrm{i}$;

)

write_serial(';');

return 1;

) 



